This item was submitted to Loughborough's Research Repository by the author.

Items in Figshare are protected by copyright, with all rights reserved, unless otherwise indicated.

\title{
Scattering coefficients for a sphere in a visco-acoustic medium for arbitrary partial wave order
}

PLEASE CITE THE PUBLISHED VERSION

LICENCE

CC BY-NC 4.0

\section{REPOSITORY RECORD}

Pinfield, Valerie, Pierre Marechal, and M Mahbub Alam. 2020. "Scattering Coefficients for a Sphere in a Viscoacoustic Medium for Arbitrary Partial Wave Order". Loughborough University.

https://doi.org/10.17028/rd.lboro.12173667.v2. 


\begin{abstract}
$>$ Analytical solution for the scattering coefficients for n'th order
$>$ using the nonthermal boundary equation matrix

$>$ to derive coefficients Tn for compressional and shear modes

$>$ for small compressional wavenumber kca

$>$ by Valerie Pinfield, Loughborough University, April 2020

$>$ In Maple 2015.0 (Maplesoft)

$>$ Paper submitted to WaveMotion 2020.

$>$ "Scattering coefficients for a sphere in a visco-acoustic medium for arbitrary partial wave order"

> by M. Mahbub Alam, Valerie J. Pinfield, Pierre Maréchal

$>$ Using an incident potential for both compressional or shear incident wave of partial wave type jn (kr)Pn(costheta)

$>$ The incident shear wave is taken to be of the form $\mathrm{u}=\operatorname{curl}$ curl $\left(\mathrm{X}^{*} \mathrm{r}^{*} \mathrm{er}\right)$ where er is unit vector in radial direction.

$>$ The potential $X$ is taken to be of plane wave type i.e. $X=\operatorname{sigma}(n) i^{\wedge} n(2 n+1) j n(k s r) \operatorname{Pn}(\operatorname{costheta})$

$>$ Note that this is not a planar shear incident wave in displacement or velocity.

Using $\mathrm{u}=+\operatorname{grad}(\mathrm{phi})$ convention for compressional waves (so opposite to Pinfield's papers with Forrester)

All coefficients are scaled by the relevant bessel or hankel function to remove factors of $\mathrm{x}^{\wedge} \mathrm{n}$ and the incident wave potential is also scaled.
\end{abstract}

Equations are in the order $\mathrm{u} r \mathrm{r}, \mathrm{u}$ _theta, sigma_rr, sigma_rtheta ( $\mathrm{u}$ is displacement, sigma is stress) Coefficients are in the order TnI $\bar{C}$, TnIS, BnI $\bar{C}$, BnIS where

$\mathrm{T}$ are outside coefficients, $\mathrm{B}$ are inside coefficients, and I represents incident wave type

SCALE all wave potentials so that the terms in $\mathrm{x}^{\wedge} \mathrm{n}$ are removed.

Scaling is as follows:

incident waves divided by jn(kIa)where I indicates incident wave type $\mathrm{C}$ or $\mathrm{S}$

TnIC $=$ TnICscaled*jn(kIa)/hn(kca)

TnIS $=$ TnISscaled $*$ jn $(\mathrm{kIa}) / \mathrm{hn}(\mathrm{ksa})$

so with coefficients matrix in order (TnIC,TnIS,BnIC,BnIS) the columns are scaled as:

$(/ \mathrm{hn}(\mathrm{kca}) / \mathrm{hn}(\mathrm{ksa}) / \mathrm{jn}(\mathrm{kc} \mathrm{a}) / \mathrm{jn}(\mathrm{ks} \mathrm{a}))$

and incident matrix RHSC is /jn(kca) and RHSS is /jn(ksa)

(primed denotes inside scatterer)

Initially make no assumptions about magnitude of ys', later expand solution as series in ys'

This is because the $n=1$ results seem to come from the second term in series in ys' (kspa) in numerator and denominator

So obtain the leading order term in a series in kca, and then take a series in kspa of numerator and denominator

Solutions for the $n=1$ coefficient were presented in a previous paper Pinfield and Forrester, Journal of the Acoustical Society of America, 141, 649 (2017); doi: 10.1121/1.4974142. These results have been used to identify simplifications for the formulae for general $\mathrm{n}$ here.

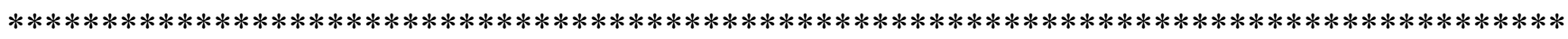

$* * * * * * * * * * * * * * * *$

Set up boundary equation in matrix form. Notation: kca,ksa,kcpa,kspa are wavenumbers $\mathrm{x}$ radius for compressional, shear outside then inside

jnp_jnk ${ }^{* *}$ a and similarly for hnp_hn are ratios of jn' $\left(\mathrm{k}^{* *} \mathrm{a}\right) / \mathrm{jn}\left(\mathrm{k}^{* *} \mathrm{a}\right)$ etc. RhoC and rhoP are densities in embeedding medium and particle respectively

\footnotetext{
I> restart;

> with(LinearAlgebra):
} 


$$
\begin{aligned}
& \lfloor>\operatorname{LHS}:=\operatorname{Matrix}(4,4): \\
& \text { [> RHSC := Matrix }(4,1): \text { incident compressional wave } \\
& \text { [> RHSS := Matrix }(4,1): \text { \# incident shear wave } \\
& \text { ¿ RHSS: } \\
& \text { [> } \operatorname{LHS}(1,1):=k c a \cdot h n p \_h n k c a: \\
& {[>\operatorname{LHS}(2,1):=1 \text { : }} \\
& >\operatorname{LHS}(3,1):=\frac{r h o C}{(k s a \cdot k s a)} \cdot\left((k s a \cdot k s a-2 \cdot n \cdot(n+1))+4 \cdot k c a \cdot h n{ }_{-} h n k c a\right): \\
& {\left[>\operatorname{LHS}(4,1):=\frac{2 \cdot r h o C}{k s a \cdot k s a} \cdot\left(1-k c a \cdot h n p \_h n k c a\right):\right.} \\
& {[>\operatorname{LHS}(1,2):=n \cdot(n+1):} \\
& {\left[>\operatorname{LHS}(2,2):=\left(1+k s a \cdot h n p \_h n k s a\right):\right.} \\
& {\left[>\operatorname{LHS}(3,2):=\frac{2 \cdot n \cdot(n+1) \cdot r h o C}{k s a \cdot k s a} \cdot\left(1-k s a \cdot h n p \_h n k s a\right):\right.} \\
& {\left[>\operatorname{LHS}(4,2):=\frac{r h o C}{k s a \cdot k s a} \cdot\left((k s a \cdot k s a-2 \cdot n \cdot(n+1)+2)+2 \cdot k s a \cdot h n p \_h n k s a\right):\right.} \\
& {\left[>\operatorname{LHS}(1,3):=-k c p a \cdot j n p \_j n k c p a:\right.} \\
& \text { [> } \\
& {[>\operatorname{LHS}(2,3):=-1 \text { : }} \\
& >\operatorname{LHS}(3,3):=-\frac{r h o P}{k s p a \cdot k s p a} \cdot\left((k s p a \cdot k s p a-2 \cdot n \cdot(n+1))+4 \cdot k c p a \cdot j n p \_j n k c p a\right): \\
& {\left[>\operatorname{LHS}(4,3):=-\frac{2 \cdot \operatorname{rhoP}}{k s p a \cdot k s p a} \cdot(1-k c p a \cdot j n p j \text { jnkcpa }):\right.}
\end{aligned}
$$

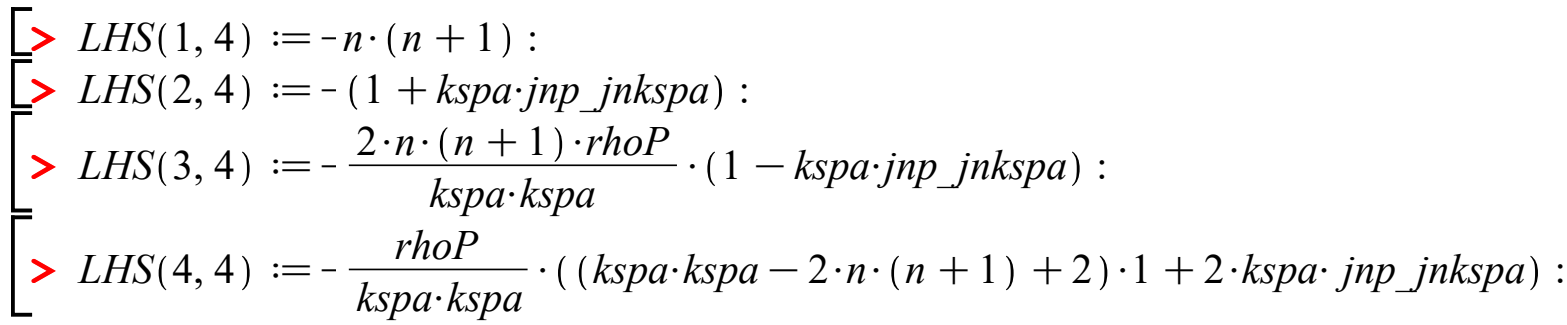

$\left[>\operatorname{RHSC}(1,1):=-k c a \cdot j n p \_j n k c a: \#\right.$ for incident compressional waves

$>\operatorname{RHSC}(2,1):=-1$ :

$\left[>\operatorname{RHSC}(3,1):=-\left(\frac{r h o C}{k s a \cdot k s a}\right) \cdot\left((k s a \cdot k s a-2 \cdot n \cdot(n+1))+4 \cdot k c a \cdot j n p \_j n k c a\right)\right.$ :

$\left[>\operatorname{RHSC}(4,1):=2 \frac{r h o C}{k s a \cdot k s a} \cdot(k c a \cdot j n p j j k c a-1):\right.$

$$
\begin{aligned}
& {[>\operatorname{RHSS}(1,1):=-n \cdot(n+1): \# \text { for incident shear wave }} \\
& {\left[>\operatorname{RHSS}(2,1):=-\left(1+k s a \cdot j n p \_j n k s a\right):\right.} \\
& >\operatorname{RHSS}(3,1):=2 \cdot \frac{r h o C}{k s a \cdot k s a} \cdot n \cdot(n+1) \cdot\left(k s a \cdot j n p \_j n k s a-1\right): \\
& {\left[>\operatorname{RHSS}(4,1):=-\frac{r h o C}{k s a \cdot k s a} \cdot\left((k s a \cdot k s a-2 \cdot n \cdot(n+1)+2)+2 \cdot k s a \cdot j n p \_j n k s a\right)\right. \text { : }}
\end{aligned}
$$


Expand the Bessel and Hankel functions in the compressional wavenumbers for small arguments and find the ratios of jnp/jn etc. using recurrence relations to work with scaled boundary equations

[Check the series expansions for hnx

$>x h n \_$exp $:=i^{(-n-1)} \cdot \frac{(2 \cdot n) ! \cdot i^{(n)}}{n ! \cdot(2 \cdot x)^{n}} \cdot\left(1-i \cdot x-\frac{(n-1)}{(2 \cdot n-1)} \cdot x^{2}\right) \# \frac{\text { this is } h n(x) \cdot x}{\exp (i \cdot x)}$ for $n$

$$
\geq 2 O N L Y
$$

$$
x h n \_\exp :=\frac{i^{-n-1}(2 n) ! i^{n}\left(1-i x-\frac{(n-1) x^{2}}{2 n-1}\right)}{n !(2 x)^{n}}
$$

(it works for $n=1$ but the $x^{2}$ term in the bracket is identically zero in that case since $(\mathrm{n}-1)=0$

. Obtained from the Epstein and Carhart (1953)

$(E \& C)$ paper expressions for hn at small arguments

$$
\begin{aligned}
& >x h n 1 \_\exp :=\operatorname{subs}\left(n=n+1, x h n \_\exp \right) \# \frac{h n+1(x) \cdot x}{\exp (i \cdot x)} \\
& x h n 1 \_\exp :=\frac{i^{-n-2}(2 n+2) ! i^{n+1}\left(1-i x-\frac{n x^{2}}{2 n+1}\right)}{(n+1) !(2 x)^{n+1}} \\
& >\text { hn1_hnRatio }:=\operatorname{simplify}\left(\frac{x h n 1 \_\exp }{x h n \_\exp }\right) \# \text { the ratio of } h n+1 \text { over hn for small arguments } x \\
& \text { hn1_hnRatio }:=\frac{(2 n-1)\left(2 i n x+n x^{2}+i x-2 n-1\right)}{x\left(2 i n x+n x^{2}-i x-x^{2}-2 n+1\right)}
\end{aligned}
$$

$[>$ hn1_hnxx $:=\operatorname{simplify}(\operatorname{simplify}(\operatorname{series}($ hn1_hnRatio, $x, 2)))$;

\#series expression for the ratio of $h n \overline{+1}$ over hn for small argments $x$

$$
h n 1_{-} h n x x:=\frac{2 n+1}{x}+\mathrm{O}(x)
$$

Now obtain the ratio of the derivative to the Hankel function i.e. $\mathrm{hn} / \mathrm{hn}$ using the recurrence relation $\mathrm{Rn}^{\prime} / \mathrm{Rn}=(\mathrm{n} / \mathrm{x})-\mathrm{Rn}+1 / \mathrm{Rn}$

$>h n p \_h n x x:=\frac{n}{x} \cdot\left(1-\frac{h n 1 \_h n x x \cdot x}{n}\right)$; \#ratio of $\frac{h n^{\prime}}{h n}$ using recurrence relation

$$
h n p_{-} h n x x:=\frac{n\left(1-\frac{\left(\frac{2 n+1}{x}+\mathrm{O}(x)\right) x}{n}\right)}{x}
$$

> hnp_hnxx $:=\operatorname{simplify}(\operatorname{series}($ hnp_hnxx, $x, 3))$

$$
\text { hnp_hnxx:= } \frac{-n-1}{x}+\mathrm{O}(x)
$$

Now do the same for the Bessel functions - this is easier because they can be expanded easily as series in $\mathrm{x}$ - see E\&C

$\left\lceil\right.$ jnxx $:=\frac{x^{n}}{f a c_{j} j n} \cdot\left(1-\frac{x^{2}}{2 \cdot(2 \cdot n+3)}+\frac{x^{4}}{2 \cdot 4 \cdot(2 \cdot n+3) \cdot(2 \cdot n+5)}+\mathrm{O}\left(x^{6}\right)\right)$ 


$$
\begin{aligned}
& \text { \#extend this to the 4th order term in bracket for the assumption of general kspa - it is } \\
& j n x x:=\frac{x^{n}\left(1-\frac{x^{2}}{4 n+6}+\frac{1}{8} \frac{x^{4}}{(2 n+3)(2 n+5)}+\mathrm{O}\left(x^{6}\right)\right)}{f a c \_j n} \\
& >j n 1 x x:=\frac{\operatorname{subs}(n=n+1, j n x x)}{(2 n+3)} \\
& \# j n+1(x) \text { - do a manual adjustment on the factor fac_jn which is } 1 \cdot 3 \cdot \ldots(2 n+1) \\
& j n 1 x x:=\frac{x^{n+1}\left(1-\frac{x^{2}}{4 n+10}+\frac{1}{8} \frac{x^{4}}{(2 n+5)(2 n+7)}+\mathrm{O}\left(x^{6}\right)\right)}{f a c \_j n(2 n+3)} \\
& >\text { jn1_jnxx }:=\operatorname{simplify}\left(\left(\frac{j n 1 x x}{j n x x}\right)\right) \\
& j n 1 \text { jnxx }:=\left(\left(x^{4}+32 \mathrm{O}\left(x^{6}\right) n^{2}-8 n x^{2}+192 \mathrm{O}\left(x^{6}\right) n+32 n^{2}-28 x^{2}+280 \mathrm{O}\left(x^{6}\right)+192 n\right.\right. \\
& +280) x) /\left(\left(x^{4}+32 \mathrm{O}\left(x^{6}\right) n^{2}-8 n x^{2}+128 \mathrm{O}\left(x^{6}\right) n+32 n^{2}-20 x^{2}+120 \mathrm{O}\left(x^{6}\right)\right.\right. \\
& +128 n+120)(2 n+7)) \\
& >\operatorname{simplify}\left(\operatorname{series}\left(j n 1 \_j n x x, x, 4\right)\right) \\
& \frac{1}{2 n+3} x+\frac{1}{(2 n+3)\left(4 n^{2}+16 n+15\right)} x^{3}+\mathrm{O}\left(x^{5}\right) \\
& > \\
& >\text { jnp_jnxx }:=\operatorname{simplify}\left(\operatorname{series}\left(\frac{n}{x} \cdot\left(1-\frac{j n 1 \_j n x x \cdot x}{n}\right), x, 5\right)\right) \\
& \text { jnp_jnxx : }=\frac{n}{x}-\frac{1}{2 n+3} x-\frac{1}{(2 n+3)\left(4 n^{2}+16 n+15\right)} x^{3}+\mathrm{O}\left(x^{5}\right)
\end{aligned}
$$

Now substitute the wavenumber-radius products into the relevant Bessel/Hankel functions ONLY for compressional modes for which $\mathrm{kca}<<1$ and $\mathrm{kcpa}<<1$

Do not make any assumption about the magnitude of the shear wavenumber-radius products in the continuous phase

Also use kcpa=kca*kcp_kc since kcp and kc are the same order of magnitude.

FFirst for compressional waves outside particles

> hnp_hnkca $:=\operatorname{series}\left(\operatorname{subs}\left(x=k c a, h n p \_h n x x\right), k c a, 3\right)$;

$$
\text { hnp_hnkca: }=\frac{-n-1}{k c a}+\mathrm{O}(k c a)
$$

for compressional waves inside particles

$\left[>\right.$ jnp_jnkcpa $:=\operatorname{series}\left(\operatorname{subs}\left(x=k c a \cdot k c p \_k c, j n p \_j n x x\right), k c a, 3\right)$;

$$
j n p \_j n k c p a:=\frac{\frac{n}{k c p \_k c}}{k c a}-\frac{k c p \_k c}{2 n+3} k c a+\mathrm{O}\left(k c a^{3}\right)
$$

For compressional incident waves

$\left[>\right.$ jnp_jnkca $:=\operatorname{series}\left(\operatorname{subs}\left(x=k c a, j n p \_j n x x\right), k c a, 3\right)$

$$
\text { jnp_jnkca }:=\frac{n}{k c a}-\frac{1}{2 n+3} k c a+\mathrm{O}\left(k c a^{3}\right)
$$


$-k s a^{4} k s p a^{5} r h o C^{2} j n p \_j n k s p a+k s a^{4} k s p a^{5}$ rhoC rhoP jnp_jnkspa

$-4 k s a^{4} k s p a^{3} n^{2}$ rhoC rhoP jnp_jnkspa $-4 k s a^{4} k s p a n^{4}$ rhoP $P^{2}$ jnp_jnkspa

$-2 \mathrm{ksa}^{3} \mathrm{kspa}^{5}$ rho $\mathrm{C}^{2} \mathrm{hnp} \_$hnksajnp_jnkspa $+2 \mathrm{ksa}^{3} \mathrm{kspa} a^{5}$ rhoC rhoP hnp_hnksajnp_jnkspa

$-4 k s a^{3} k s p a^{4} n^{2}$ rhoC rhoP hnp_hnksa $-8 k s a^{3} k s p a^{3} n^{2}$ rhoC rhoP hnp_hnksajnp_jnkspa

$+8 \mathrm{ksa}^{3} \mathrm{kspa}^{2} \mathrm{n}^{4}$ rhoC rhoP hnp_hnksa $+4 \mathrm{ksa}^{2} \mathrm{kspa} \mathrm{a}^{5} \mathrm{n}^{2} \mathrm{rhoC} \mathrm{C}^{2} \mathrm{jnp}$ jnkspa

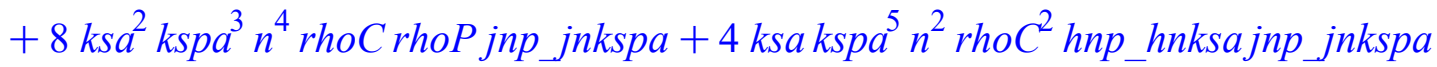

$-4 \mathrm{ksakspa} \mathrm{n}^{4}$ rho $C^{2}$ hnp_hnksa $-4 k s p a^{5} n^{4}$ rho $C^{2}$ jnp_jnkspa

$-2 \mathrm{ksa}^{5} \mathrm{kspa}^{2} \mathrm{n}$ rhoC rhoP hnp_hnksa $-8 \mathrm{ksa}^{5} \mathrm{kspa} n \mathrm{rhoP} \mathrm{P}^{2} \mathrm{hnp}$ hnksajnp_jnkspa

$-4 k s a^{5} n^{3}$ rhoP $P^{2}$ hnp_hnksa $+k s a^{4} k s p a^{4} n r h o C^{2}-2 k s a^{4} k s p a^{4} n$ rhoC rhoP

$+k s a^{4} k s p a^{4} n r h o P^{2}+2 k s a^{4} k s p a^{3} n r h o P^{2} j n p \_j n k s p a+4 k s a^{4} k s p a^{2} n^{3}$ rhoC rhoP

$-4 k s a^{4} k s p a^{2} n^{3} r h o P^{2}-4 k s a^{4} k s p a n^{3} r h o P^{2} j n p j j n k p a+4 k s a^{4} n^{5} r h o P^{2}$

$+2 \mathrm{ksa}^{3} \mathrm{kspa}^{4} \mathrm{n}$ rho $\mathrm{C}^{2}$ hnp_hnksa $+16 \mathrm{ksa}^{3} \mathrm{kspa}^{3} \mathrm{n}$ rhoC rhoP hnp_hnksajnp_jnkspa

$+8 k s a^{3} k s p a^{2} n^{3}$ rhoC rhoP hnp_hnksa $-2 k s a^{2} k s p a^{5} n$ rhoC rhoP jnp_jnkspa

$-4 k s a^{2} k s p a^{4} n^{3} r h o C^{2}+4 k s a^{2} k s p a^{4} n^{3}$ rhoC rhoP $+8 k s a^{2} k s p a^{3} n^{3}$ rhoC rhoP jnp_jnkspa

$-8 k s a^{2} k s p a^{2} n^{5}$ rhoC rhoP - $8 k s a k s p a^{5} n$ rhoC $C^{2} h n$ _hnksajnp_jnkspa

$-4 k s a k s p a^{4} n^{3}$ rho $C^{2} h n p \_h n k s a-4 k s p a^{5} n^{3} r h o C^{2} j n p \_j n k s p a+4 k s p a^{4} n^{5}$ rho $C^{2}$

$-2 k s a^{5} k s p a^{2}$ rho $P^{2}$ hnp_hnksa $+8 k s a^{5} n^{2} r h o P^{2} h n p \_h n k s a$

$+2 k s a^{4} k s p a^{3}$ rhoC rhoP jnp_jnkspa $+8 k s a^{4} k s p a n^{2} r h o P^{2} j n p \_j n k p a+4 k s a^{4} n^{4} r h o P^{2}$

$+2 \mathrm{ksa}^{3} \mathrm{kspa} \mathrm{a}^{4}$ rhoCrhoP hnp_hnksa $-16 \mathrm{ksa}^{3} \mathrm{kspa} \mathrm{a}^{2} \mathrm{n}^{2}$ rhoCrhoP hnp_hnksa

$-2 k s a^{2} k s p a^{5} r h o C^{2} j n p \_j n k s p a-16 k s a^{2} k s p a^{3} n^{2}$ rhoC rhoP jnp_jnkspa

$-8 \mathrm{ksa}^{2} \mathrm{kspa} \mathrm{a}^{2} \mathrm{n}^{4} \mathrm{rhoC}$ rhoP $+8 \mathrm{ksakspa} \mathrm{a}^{2} \mathrm{n}^{2} \mathrm{rhoC} \mathrm{C}^{2} \mathrm{hnp} \_$hnksa

$+8 k s p a^{5} n^{2} r h o C^{2} j n p \_j n k s p a+4 k s p a^{4} n^{4}$ rho $C^{2}-2 k s a^{4} k s p a^{2} n$ rhoC rhoP

$+2 k s a^{4} k s p a^{2} n r h o P^{2}-8 k s a^{4} n^{3} r h o P^{2}+2 k s a^{2} k s p a^{4} n r h o C^{2}-2 k s a^{2} k s p a^{4} n$ rhoC rhoP

$+16 k s a^{2} k s p a^{2} n^{3}$ rhoC rhoP $\left.\left.-8 k s p a^{4} n^{3} r h o C^{2}\right)\right) /($

-4 ksa $a^{5}$ span ${ }^{4}$ rhoP $P^{2}$ hnp_hnksajnp_jnkspa

$+8 \mathrm{ksa}^{3} \mathrm{kspa}^{3} \mathrm{n}^{4}$ rhoC rhoP hnp_hnksajnp_jnkspa

-4 ksa kspa ${ }^{5} n^{4}$ rhoC ${ }^{2}$ hnp_hnksajnp_jnkspa $+k s a^{5} k s p a^{4} n$ rhoCrhoP hnp_hnksa

$+k s a^{5} k s p a^{4} n$ rhoP $P^{2}$ hnp_hnksa $+2 k^{2} a^{5} k_{s p a} a^{3} n$ rhoC rhoP hnp_hnksajnp_jnkspa

$+2 k s a^{5} k s p a^{3} n r h o P^{2} h n p \_h n k s a j n p \_j n k s p a-4 k s a^{5} k s p a^{2} n^{3} r h o P^{2} h n p \_h n k s a$

$-8 k s a^{5} k s p a n^{3} r h o P^{2} h n p \_h n k s a j n p \_j n k s p a+4 k s a^{5} n^{5} r h o P^{2}$ hnp_hnksa

- $k s a^{4} k s p a^{5} n$ rho $C^{2}$ jnp_jnkspa - ksa $a^{4} s p a^{5}$ n rhoC rhoP jnp_jnkspa

$-4 k s a^{4} k s p a^{3} n^{3}$ rhoC rhoP jnp_jnkspa $-4 k s a^{4} k s p a n^{5} r h o P^{2} j n p \_j n k s p a$

$-2 \mathrm{ksa}^{3} \mathrm{kspa}^{5}$ n rhoC ${ }^{2}$ hnp_hnksajnp_jnkspa

$-2 k s a^{3} k s p a^{5} n$ rhoC rhoP hnp_hnksajnp_jnkspa $+4 k s a^{3} k s p a^{4} n^{3}$ rhoC rhoP hnp_hnksa

$+16 \mathrm{ksa}^{3} \mathrm{kspa}^{3} \mathrm{n}^{3}$ rhoC rhoP hnp_hnksajnp_jnkspa $-8 \mathrm{ksa} a^{3} \mathrm{kspa}^{2} \mathrm{n}^{5}$ rhoC rhoP hnp_hnksa

$+4 k s a^{2} k s p a^{5} n^{3} r h o C^{2} j n p \_j n k s p a+8 k s a^{2} k s p a^{3} n^{5}$ rhoC rhoP jnp_jnkspa 
$-8 k s a k s p a^{5} n^{3} r h o C^{2} h n p \_h n k s a j n p \_j n k s p a+4 k s a k s p a^{4} n^{5} r h o C^{2} h n p \_h n k s a$

$-4 k s p a^{5} n^{5} r h o C^{2} j n p \_j n k s p a+k s a^{5} k s p a^{4} r h o P^{2} h n p \_h n k s a$

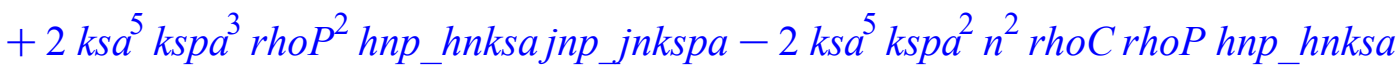

$-4 k s a^{5} k s p a^{2} n^{2} r h o P^{2} h n p \_h n k s a+4 k s a^{5} k s p a n^{2} r h o P^{2} h n p \_h n k s a j n p \_j n k s p a$

$+8 k s a^{5} n^{4}$ rhoP $P^{2}$ hnp_hnksa $-k s a^{4} k s p a^{5}$ rhoC rhoP jnp jnkspa $+k s a^{4} k s p a^{4} n^{2} r h o C^{2}$

$-2 k s a^{4} k s p a^{4} n^{2}$ rhoC rhoP $+k s a^{4} k s p a^{4} n^{2} r h o P^{2}-8 k s a^{4} k s p a^{3} n^{2}$ rhoC rhoP jnp jnkspa

$+2 k s a^{4} k s p a^{3} n^{2} r h o P^{2} j n p \_j n k s p a+4 k s a^{4} k s p a^{2} n^{4}$ rhoC rhoP $-4 k s a^{4} k s p a^{2} n^{4} r h o P^{2}$

$-12 k s a^{4} k s p a n^{4} r h o P^{2} j n p$ jnkspa $+4 k s a^{4} n^{6} r h o P^{2}$

$-2 \mathrm{ksa}^{3} \mathrm{kspa} \mathrm{a}^{5}$ rhoC rhoP hnp_hnksajnp_jnkspa $+2 \mathrm{ksa} a^{3} k s p a^{4} n^{2} r h o C^{2} h n p \_h n k s a$

$+4 \mathrm{ksa}^{3} \mathrm{kspa}^{4} \mathrm{n}^{2}$ rhoC rhoP hnp_hnksa $-8 \mathrm{ksa}^{3} \mathrm{kspa}^{3} \mathrm{n}^{2}$ rhoC rhoP hnp_hnksajnp_jnkspa

$-16 k s a^{3} k s p a^{2} n^{4}$ rhoC rhoP hnp_hnksa $+8 k s a^{2} k s p a^{5} n^{2} r h o C^{2} j n p \_j n k s p a$

$-2 k s a^{2} k s p a^{5} n^{2}$ rhoC rhoP jnp_jnkspa $-4 k s a^{2} k s p a^{4} n^{4} r h o C^{2}+4 k s a^{2} k s p a^{4} n^{4}$ rhoC rhoP

$+24 \mathrm{ksa}^{2} \mathrm{kspa}^{3} \mathrm{n}^{4}$ rhoC rhoP jnp_jnkspa $-8 \mathrm{ksa}^{2} \mathrm{kspa} \mathrm{n}^{6} \mathrm{n}^{6} \mathrm{rhoC}$ rhoP

$+4 k s a k s p a^{5} n^{2} r h o C^{2} h n p \_h n k s a j n p \_j n k s p a+8 k s a k s p a^{4} n^{4} r h o C^{2} h n p \_h n k s a$

$-12 k s p a^{5} n^{4} r h o C^{2} j n p \_j n k s p a+4 k s p a^{4} n^{6} r h o C^{2}+2 k s a^{5} k s p a^{2} n r h o P^{2} h n p \_h n k s a$

$+8 k s a^{5}$ kspa $n$ rhoP $P^{2}$ hnp_hnksajnp_jnkspa $-4 k s a^{5} n^{3}$ rhoP $P^{2}$ hnp_hnksa

$-2 \mathrm{ksa}^{4} \mathrm{kspa} \mathrm{a}^{4} \mathrm{n}$ rhoC rhoP $+2 \mathrm{ksa} a^{4} \mathrm{kspa} a^{4} n$ rho $P^{2}-2 k s a^{4} k s p a^{3} n$ rhoC rhoP jnp_jnkspa

$+4 k s a^{4} k s p a^{3} n r h o P^{2} j n p \_j n k s p a+8 k s a^{4} k s p a^{2} n^{3}$ rhoC rhoP $-8 k s a^{4} k s p a^{2} n^{3}$ rho $P^{2}$

$-4 k s a^{4} k s p a n^{3} r h o P^{2} j n p \_j n k s p a+12 k s a^{4} n^{5} r h o P^{2}$

$-2 \mathrm{ksa}^{3} \mathrm{kspa}^{4} \mathrm{n}$ rhoC rhoP hnp_hnksa $-16 \mathrm{ksa}^{3} \mathrm{kspa}^{3} \mathrm{n}$ rhoC rhoP hnp_hnksajnp_jnkspa

$+8 k s a^{3} k_{s p a} n^{3}$ rhoCrhoP hnp_hnksa $+2 k_{s a} a^{2} k s p a^{5} n$ rho $C^{2}$ jnp jnkspa

$-4 k s a^{2} k s p a^{5} n$ rhoC rhoP jnp_jnkspa $-8 k s a^{2} k s p a^{4} n^{3} r h o C^{2}+8 k s a^{2} k s p a^{4} n^{3}$ rhoC rhoP

$+8 \mathrm{ksa}^{2} \mathrm{kspa}^{3} \mathrm{n}^{3}$ rhoC rhoP jnp_jnkspa $-24 \mathrm{ksa}^{2} \mathrm{kspa}^{2} \mathrm{n}^{5}$ rhoC rhoP

$+8 k s a k s p a^{5} n$ rho $C^{2} h n p \_h n k s a j n p \_j n k s p a-4 k s a k s p a^{4} n^{3}$ rho $C^{2} h n p \_h n k s a$

$-4 k s p a^{5} n^{3} r h o C^{2} j n p \_j n k s p a+12 k s p a^{4} n^{5} r h o C^{2}+2 k s a^{5} k s p a^{2} r h o P^{2} h n p \_h n k s a$

$-8 k s a^{5} n^{2} r h o P^{2} h n p \_h n k s a-k s a^{4} k s p a^{4} r h o C r h o P+k s a^{4} k s p a^{4} r h o P^{2}$

$+2 k s a^{4} k s p a^{3} r h o P^{2} j n p \_j n k s p a+2 k s a^{4} k s p a^{2} n^{2} r h o C r h o P-2 k s a^{4} k s p a^{2} n^{2} r h o P^{2}$

$+12 \mathrm{ksa}^{4} \mathrm{kspa}^{2} \mathrm{rhoP}^{2}$ jnp_jnkspa $+4 \mathrm{ksa}^{4} \mathrm{n}^{4} \mathrm{rhoP}^{2}-2 \mathrm{ksa} \mathrm{k}^{3} \mathrm{kspa} \mathrm{a}^{4} \mathrm{rhoC}$ rhoP hnp_hnksa

+ $16 \mathrm{ksa}^{3} \mathrm{kspa}^{2} \mathrm{n}^{2}$ rhoC rhoP hnp_hnksa $-2 \mathrm{ksa}^{2} \mathrm{kspa} \mathrm{a}^{5}$ rhoC rhoP jnp_jnkspa

$-2 k s a^{2} k s p a^{4} n^{2} r h o C^{2}+2 k s a^{2} k s p a^{4} n^{2}$ rhoC rhoP

$-24 k s a^{2} k s p a^{3} n^{2}$ rhoC rhoP jnp_jnkspa $-8 k s a^{2} k s p a^{2} n^{4}$ rhoC rhoP

$-8 k s a k s p a^{4} n^{2}$ rho $C^{2}$ hnp_hnksa $+12 k s p a^{5} n^{2} r h o C^{2} j n p \_j n k s p a+4 k s p a^{4} n^{4} r_{h o C^{2}}$

$+4 k s a^{4} k s p a^{2} n r h o P^{2}+8 k s a^{4} k s p a n r h o P^{2} j n p \_j n k s p a-12 k s a^{4} n^{3} r h o P^{2}$

$-4 \mathrm{ksa}^{2} \mathrm{kspa} \mathrm{a}^{4} \mathrm{n}$ rhoC rhoP - $16 \mathrm{ksa}^{2} \mathrm{kspa}^{3} \mathrm{n}$ rhoC rhoP jnp_jnkspa

$+24 k s a^{2} k s p a^{2} n^{3}$ rhoC rhoP $+8 k s p a^{5} n r h o C^{2} j n p \_j n k s p a-12 k s p a^{4} n^{3} r h o C^{2}$

$+2 k s a^{4} k s p a^{2} r h o P^{2}-8 k s a^{4} n^{2} r h o P^{2}-2 k s a^{2} k s p a^{4}$ rhoC rhoP 
$+16 k s a^{2} k s p a^{2} n^{2}$ rhoC rhoP $\left.-8 k s p a^{4} n^{2} r h o C^{2}\right)$

$>$ TnCCLeadNum $:=$ numer $($ TnCCLeadkca_) :

\#this is not giving me the numerator - instead, manually copy the output above to enter the numerator and denominator

$>$ numTnCC_: $=-n \cdot\left(4 \mathrm{ksa}^{5} \mathrm{kspan} \mathrm{n}^{3} \mathrm{rhoP^{2 }}\right.$ hnp_hnksajnp_jnkspa

$-8 \mathrm{ksa}^{3} \mathrm{kspa}^{3} \mathrm{n}^{3}$ rhoC rhoP hnp_hnksajnp_jnkspa

$+4 k s a k s p a^{5} n^{3}$ rhoC ${ }^{2}$ hnp_hnksajnp_jnkspa $+k s a^{5}$ kspa $a^{4}$ rhoCrhoP hnp_hnksa

- ksa ${ }^{5} k s p a^{4} r h o P^{2} h n p \_h n k s a+2 k s a^{5} k s p a^{3}$ rhoC rhoP hnp_hnksajnp_jnkspa

$-2 k s a^{5} k s p a^{3}$ rhoP $P^{2}$ hnp_hnksajnp_jnkspa $+4 k s a^{5} k s p a^{2} n^{2}$ rhoP $P^{2}$ hnp_hnksa

$+4 k s a^{5} k s p a n^{2} r h o P^{2}$ hnp_hnksajnp_jnkspa $-4 k s a^{5} n^{4} r h o P^{2} h n p \_h n k s a$

$-k s a^{4} k s p a^{5} r h o C^{2} j n p \_j n k s p a+k s a^{4} k s p a^{5}$ rhoC rhoP jnp_jnkspa

$-4 k s a^{4} k s p a^{3} n^{2}$ rhoC rhoP jnp_jnkspa $-4 k s a^{4} k s p a n^{4}$ rho $P^{2}$ jnp_jnkspa

$-2 \mathrm{ksa}^{3} \mathrm{kspa} a^{5}$ rho ${ }^{2}$ hnp_hnksajnp_jnkspa $+2 \mathrm{ksa}^{3} \mathrm{kspa} a^{5}$ rhoC rhoP hnp_hnksajnp_jnkspa

$-4 \mathrm{ksa}^{3} \mathrm{kspa}^{4} \mathrm{n}^{2}$ rhoC rhoP hnp_hnksa $-8 \mathrm{ksa}^{3} \mathrm{kspa} \mathrm{a}^{3} \mathrm{n}^{2}$ rhoC rhoP hnp_hnksajnp_jnkspa

$+8 k s a^{3} k s p a^{2} n^{4}$ rhoC rhoP hnp_hnksa $+4 k s a^{2} k s p a^{5} n^{2} r h o C^{2} j n p \_j n k s p a$

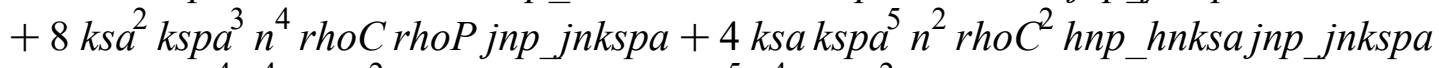

$-4 \mathrm{ksa} k s p a^{4} n^{4}$ rho $C^{2}$ hnp_hnksa $-4 \mathrm{kspa}^{5} n^{4} \mathrm{rhoC}^{2}$ jnp_jnkspa

$-2 \mathrm{ksa}^{5} \mathrm{kspa}^{2} \mathrm{n}$ rhoC rhoP hnp_hnksa $-8 \mathrm{ksa}^{5} \mathrm{kspa} n \mathrm{rhoP} \mathrm{P}^{2}$ hnp_hnksajnp_jnkspa

$-4 k s a^{5} n^{3}$ rho $P^{2} h n p \_h n k s a+k s a^{4} k s p a^{4} n r h o C^{2}-2 k s a^{4} k s p a^{4} n$ rhoC rhoP

$+k s a^{4} k s p a^{4} n$ rho $P^{2}+2 k s a^{4} k s p a^{3} n$ rho $P^{2} j n p j n k s p a+4 k s a^{4} k s p a^{2} n^{3}$ rhoC rhoP

$-4 k s a^{4} k s p a^{2} n^{3} r h o P^{2}-4 k s a^{4} k s p a n^{3} r h o P^{2} j n p j n k s p a+4 k s a^{4} n^{5} r h o P^{2}$

$+2 \mathrm{ksa}^{3} \mathrm{kspa}^{4} \mathrm{n}$ rho $\mathrm{C}^{2} \mathrm{hnp} \_$hnksa $+16 \mathrm{ksa}^{3} \mathrm{kspa}^{3} \mathrm{n}$ rhoC rhoP hnp_hnksajnp_jnkspa

$+8 \mathrm{ksa}^{3} \mathrm{kspa}^{2} \mathrm{n}^{3}$ rhoC rhoP hnp_hnksa $-2 \mathrm{ksa}^{2} \mathrm{kspa} \mathrm{a}^{5} \mathrm{n}$ rhoC rhoP jnp_jnkspa

$-4 k s a^{2} k s p a^{4} n^{3} r h o C^{2}+4 k s a^{2} k s p a^{4} n^{3}$ rhoC rhoP $+8 k s a^{2} k s p a^{3} n^{3}$ rhoC rhoP jnp jnkspa

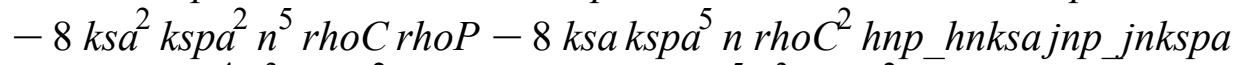

$-4 k s a k s p a^{4} n^{3} r h o C^{2} h n p \_h n k s a-4 k s p a^{5} n^{3} r h o C^{2} j n p \_j n k s p a+4 k s p a^{4} n^{5} r h o C^{2}$

$-2 k s a^{5} k s p a^{2}$ rhoP $P^{2} h n p \_h n k s a+8 k s a^{5} n^{2}$ rhoP $P^{2}$ hnp_hnksa

$+2 k_{s a}^{4} k s p a^{3}$ rhoC rhoP jnp_jnkspa $+8 k s a^{4} k s p a n^{2}$ rhoP $P^{2} j n$ jjnkspa $+4 k s a^{4} n^{4} r h o P^{2}$

$+2 \mathrm{ksa}^{3} \mathrm{kspa}^{4}$ rhoC rhoP hnp_hnksa - $16 \mathrm{ksa}^{3} \mathrm{kspa}^{2} \mathrm{n}^{2}$ rhoC rhoP hnp_hnksa

$-2 k s a^{2} k s p a^{5}$ rho $C^{2} j n p \_j n k s p a-16 k s a^{2} k s p a^{3} n^{2}$ rhoC rhoP jnp jnkspa

$-8 \mathrm{ksa}^{2} \mathrm{kspa}^{2} n^{4}$ rhoC rhoP $+8 \mathrm{ksakspa} a^{4} n^{2}$ rho $C^{2} h n p \_h n k s a+8 k s p a^{5} n^{2}$ rho $C^{2} j n p \_j n k s p a$

$+4 k s p a^{4} n^{4} r h o C^{2}-2 k s a^{4} k s p a^{2} n$ rhoC rhoP $+2 k s a^{4} k s p a^{2} n r h o P^{2}-8 k s a^{4} n^{3} r h o P^{2}$

$+2 k s a^{2} k s p a^{4} n r h o C^{2}-2 k s a^{2} k s p a^{4} n$ rhoC rhoP $+16 k s a^{2} k s p a^{2} n^{3}$ rhoC rhoP

$\left.-8 k \operatorname{spa} a^{4} n^{3} r h o C^{2}\right):$

$>$ denTnCC_ $:=-4 k s a^{5} k s p a n^{4}$ rhoP ${ }^{2}$ hnp_hnksajnp_jnkspa

$+8 \overline{k s a} a^{3} k s p a^{3} n^{4}$ rhoC rhoP hnp_hnksajnp_jnkspa

-4 ksa kspa $n^{4}$ rhoC ${ }^{2}$ hnp_hnksajnp_jnkspa $+k s a^{5}$ kspa $n$ rhoCrhoP hnp_hnksa

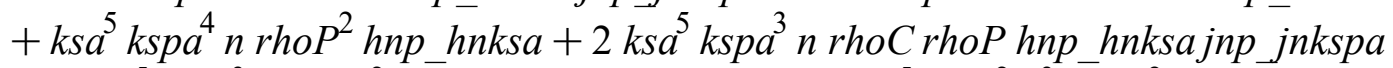

$+2 k s a^{5} k s p a^{3} n r h o P^{2} h n p \_h n k s a j n p \_j n k s p a-4 k s a^{5} k s p a^{2} n^{3}$ rhoP $P^{2} h n p \_h n k s a$

$-8 k s a^{5} k s p a n^{3}$ rho $P^{2}$ hnp_hnksajnp_jnkspa $+4 k s a^{5} n^{5}$ rho $P^{2}$ hnp_hnksa

- ksa $a^{4}$ spa $a^{5}$ rhoC ${ }^{2}$ jnp_jnkspa - ksa $a^{4} s p a^{5}$ n rhoC rhoP jnp_jnkspa

$-4 k s a^{4} k s p a^{3} n^{3}$ rhoC rhoP jnp_jnkspa $-4 k s a^{4} k s p a n^{5}$ rhoP $P^{2} j n p \_j n k s p a$ 
$-2 k s a^{3} k s p a^{5} n r h o C^{2} h n p \_h n k s a j n p \_j n k s p a$

$-2 \mathrm{ksa}^{3} \mathrm{kspa} a^{5} \mathrm{n}$ rhoC rhoP hnp_hnksajnp_jnkspa $+4 \mathrm{ksa}^{3} \mathrm{kspa} \mathrm{a}^{4} \mathrm{n}^{3}$ rhoC rhoP hnp_hnksa

$+16 k s a^{3} k s p a^{3} n^{3}$ rhoCrhoP hnp_hnksajnp_jnkspa $-8 k_{s} a^{3} k s p a^{2} n^{5}$ rhoCrhoP hnp_hnksa $+4 k s a^{2} k s p a^{5} n^{3} r h o C^{2} j n p$ jnkspa $+8 k_{s s} a^{2} k s p a^{3} n^{5}$ rhoC rhoP jnp jnkspa

$-8 k s a k s p a^{5} n^{3}$ rho $C^{2}$ hnp_hnksajnp_jnkspa $+4 k s a k s p a^{4} n^{5}$ rho $C^{2}$ hnp_hnksa

$-4 k s p a^{5} n^{5} r h o C^{2} j n p \_j n k s p a+k s a^{5} k s p a^{4} r h o P^{2} h n p \_h n k s a$

$+2 k s a^{5} k s p a^{3} r h o P^{2} h n p \_h n k s a j n p \_j n k s p a-2 k s a^{5} k s s p a^{2} n^{2}$ rhoCrhoP hnp_hnksa

$-4 \mathrm{ksa}^{5} \mathrm{kspa}^{2} \mathrm{n}^{2} \mathrm{rhoP^{2 }} h n p \_h n k s a+4 \mathrm{ksa} a^{5} \mathrm{kspa} \mathrm{n}^{2} \mathrm{rhoP^{2 }}$ hnp_hnksajnp_jnkspa

$+8 k s a^{5} n^{4} r h o P^{2} h n p \_h n k s a-k s a^{4} k s p a^{5}$ rhoC rhoP jnp_jnkspa $+k s a^{4} k s p a^{4} n^{2} r h o C^{2}$

$-2 k s a^{4} k s p a^{4} n^{2}$ rhoC rhoP $+k s a^{4} k s p a^{4} n^{2} r h o P^{2}-8 k s a^{4} k s p a^{3} n^{2}$ rhoC rhoP jnp jnkspa

$+2 k s a^{4} k s p a^{3} n^{2} r h o P^{2} j n p j n k s p a+4 k s a^{4} k s p a^{2} n^{4} r h o C r h o P-4 k s a^{4} k s p a^{2} n^{4} r h o P^{2}$

$-12 k s a^{4} k s p a n^{4} r h o P^{2} j n p$ jnkspa $+4 k s a^{4} n^{6} r h o P^{2}$

$-2 k_{s a^{3}} k s p a^{5}$ rhoC rhoP hnp_hnksajnp_jnkspa $+2 \mathrm{ksa}^{3} \mathrm{kspa}^{4} n^{2}$ rho $\mathrm{C}^{2}$ hnp_hnksa

$+4 \mathrm{ksa}^{3} \mathrm{kspa}^{4} \mathrm{n}^{2}$ rhoC rhoP hnp_hnksa $-8 \mathrm{ksa}^{3} \mathrm{kspa}^{3} \mathrm{n}^{2}$ rhoC rhoP hnp_hnksajnp_jnkspa

$-16 \mathrm{ksa}^{3} \mathrm{kspa}^{2} \mathrm{n}^{4}$ rhoCrhoP hnp_hnksa $+8 \mathrm{ksa}^{2} \mathrm{kspa}^{5} n^{2} \mathrm{rhoC}^{2}$ jnp_jnkspa

$-2 k s a^{2} k s p a^{5} n^{2}$ rhoC rhoP jnp jnkspa $-4 k s a^{2} k s p a^{4} n^{4} r h o C^{2}+4 k s a^{2} k s p a^{4} n^{4} r h o C r h o P$

$+24 \mathrm{ksa}^{2} \mathrm{kspa} a^{3} n^{4}$ rhoCrhoP jnp jnkspa $-8 \mathrm{ksa}^{2} \mathrm{kspa}^{2} n^{6}$ rhoC rhoP

$+4 k s a k s p a^{5} n^{2}$ rho $C^{2}$ hnp_hnksajnp jnkspa +8 ksa kspa ${ }^{4} n^{4}$ rhoC ${ }^{2}$ hnp hnksa

$-12 k s p a^{5} n^{4} r h o C^{2} j n p \_j n k s p a+4 k s p a^{4} n^{6} r h o C^{2}+2 k s a^{5} k s p a^{2} n$ rhoP $P^{2} h n p$ hnksa

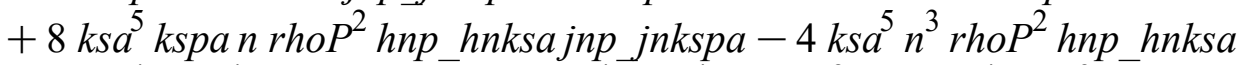

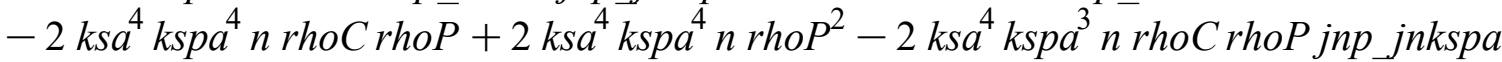

$+4 k s a^{4} k s p a^{3} n$ rhoP $P^{2}$ jnp jnkspa $+8 k s a^{4} k s p a^{2} n^{3}$ rhoC rhoP $-8 k s a^{4} k s p a^{2} n^{3} r h o P^{2}$

$-4 k s a^{4} k s p a n^{3}$ rhoP $P^{2}$ jnp jnkspa $+12 k s a^{4} n^{5} r h o P^{2}-2 k s a^{3} k s p a^{4} n$ rhoC rhoP hnp_hnksa

$-16 \mathrm{ksa}^{3} \mathrm{kspa}^{3} \mathrm{n}$ rhoCrhoP hnp_hnksa jnp_jnkspa $+8 \mathrm{ksa}^{3} \mathrm{kspa}^{2} \mathrm{n}^{3}$ rhoC rhoP hnp_hnksa

$+2 \mathrm{ksa}^{2} \mathrm{kspa} a^{5} n$ rho $\mathrm{C}^{2}$ jnp jnkspa $-4 \mathrm{ksa}^{2} \mathrm{kspa} \mathrm{a}^{5}$ n rhoC rhoP jnp jnkspa

$-8 k s a^{2} k s p a^{4} n^{3} r h o C^{2}+8 k s a^{2} k s p a^{4} n^{3}$ rhoC rhoP $+8 k s a^{2} k s p a^{3} n^{3}$ rhoC rhoP jnp jnkspa

$-24 k s a^{2} k s p a^{2} n^{5}$ rhoCrhoP $+8 k s a k s p a^{5} n$ rho $C^{2}$ hnp_hnksajnp_jnkspa

$-4 k s a k s p a^{4} n^{3} r h o C^{2} h n p \_h n k s a-4 k s p a^{5} n^{3} r h o C^{2} j n p \_j n k s p a+12 k s p a^{4} n^{5} r h o C^{2}$

$+2 k s a^{5} k s p a^{2} r h o P^{2} h n p \_h n k s a-8 k s a^{5} n^{2} r h o P^{2} h n p \_h n k s a-k s a^{4} k s p a^{4} r h o C r h o P$

$+k s a^{4} k s p a^{4} r h o P^{2}+2 k s a^{4} k s p a^{3} r h o P^{2} j n p j n k s p a+2 k s a^{4} k s p a^{2} n^{2}$ rhoC rhoP

$-2 k s a^{4} k s p a^{2} n^{2} r h o P^{2}+12 k s a^{4} k s p a n^{2} r h o P^{2} j n p$ jnkspa $+4 k s a^{4} n^{4} r h o P^{2}$

$-2 \mathrm{ksa}^{3} \mathrm{kspa} \mathrm{a}^{4} \mathrm{rhoC}$ rhoP hnp hnksa $+16 \mathrm{ksa}^{3} \mathrm{kspa}^{2} \mathrm{n}^{2}$ rhoC rhoP hnp_hnksa

$-2 \mathrm{ksa}^{2} k s p a^{5}$ rhoC rhoP jnp jnkspa $-2 \mathrm{ksa}^{2} k s p a^{4} n^{2} r h o C^{2}+2 k s a^{2} k_{s p a} n^{2}$ rhoC rhoP

$-24 k s a^{2} k s p a^{3} n^{2}$ rhoCrhoP jnp jnkspa $-8 k s a^{2} k s p a^{2} n^{4}$ rhoC rhoP

$-8 k s a k s p a^{4} n^{2} r h o C^{2} h n p \_h n k s a+12 k s p a^{5} n^{2} r h o C^{2} j n p$ jnkspa $+4 k s p a^{4} n^{4} r h o C^{2}$

$+4 k s a^{4} k s p a^{2} n r h o P^{2}+8 k s a^{4} k s p a n$ rhoP ${ }^{2}$ jnp jnkspa $-12 k s a^{4} n^{3} r h o P^{2}$

$-4 \mathrm{ksa}^{2} \mathrm{kspa}^{4} \mathrm{n}$ rhoC rhoP $-16 \mathrm{ksa}^{2} \mathrm{kspa} \mathrm{a}^{3} \mathrm{n}$ rhoC rhoP jnp jnkspa

$+24 \mathrm{ksa}^{2} k s p a^{2} n^{3}$ rhoCrhoP $+8 k s p a^{5} n$ rho $C^{2}$ jnp jnkspa $-12 k s p a^{4} n^{3} r h o C^{2}$

$+2 k s a^{4} k s p a^{2} r h o P^{2}-8 k s a^{4} n^{2} r h o P^{2}-2 k s a^{2} k s p a^{4}$ rhoC rhoP

$+16 \mathrm{ksa}^{2} \mathrm{kspa}^{2} \mathrm{n}^{2}$ rhoCrhoP $-8 \mathrm{kspa}^{4} \mathrm{n}^{2} \mathrm{rhoC}^{2}$ :

Need to get these leading terms (in kca) numerator and denominator for every coefficient BEFORE expanding as series in kspa for numerator and denominator separately. 
$>$ TnCSLeadkca_ $:=(\operatorname{series}($ 'leadterm' $(($ TnCS_ $)), k c a, 1))$

\#initially show output, then copy its numerator and denominator, then hide output

TnCSLeadkca_: $=\left(4 \mathrm{ksa}^{4} \mathrm{kspa}^{3} \mathrm{n}^{2}\right.$ rhoC rhoP jnp_jnkspa $+8 \mathrm{ksa}^{4} \mathrm{kspa}^{4} \mathrm{rhoP}^{2}$ jnp_jnkspa

$-4 k s a^{2} k s p a^{5} n^{2}$ rho $C^{2}$ jnp_jnkspa - $16 \mathrm{ksa}^{2} \mathrm{kspa}{ }^{3} n^{4}$ rhoC rhoP jnp_jnkspa

$+8 k s p a^{5} n^{4} r h o C^{2} j n p \_j n k s p a+2 k s a^{4} k s p a^{4} n$ rhoC rhoP $-2 k s a^{4} k s p a^{4} n r h o P^{2}$

$+2 \mathrm{ksa}^{4} \mathrm{kspa}^{3}$ n rhoC rhoP jnp_jnkspa $-4 \mathrm{ksa}^{4} \mathrm{kspa}^{3} \mathrm{n} r h o P^{2}$ jnp_jnkspa

$-4 k s a^{4} k s p a^{2} n^{3}$ rhoC rhoP $+8 k s a^{4} k s p a^{2} n^{3} r h o P^{2}+12 k s a^{4} k s p a n^{3} r h o P^{2} j n p \_j n k s p a$

$-8 k s a^{4} n^{5} r h o P^{2}-2 k s a^{2} k s p a^{5} n r h o C^{2} j n p \_j n k s p a+4 k s a^{2} k s p a^{5} n$ rhoC rhoP jnp_jnkspa

$+4 k s a^{2} k s p a^{4} n^{3} r h o C^{2}-8 k s a^{2} k s p a^{4} n^{3}$ rhoC rhoP

$-24 k s a^{2} k s p a^{3} n^{3}$ rhoC rhoP jnp jnkspa $+16 k s a^{2} k s p a^{2} n^{5}$ rhoC rhoP

$+12 k s p a^{5} n^{3}$ rho $C^{2} j n p j n k s p a-8 k s p a^{4} n^{5}$ rho $C^{2}+k s a^{4} k s p a^{4}$ rhoC rhoP

$-k s a^{4} k s p a^{4}$ rho $P^{2}-2 k s a^{4} k s p a^{3}$ rhoP $P^{2} j p$ jnkspa $-2 k s a^{4} k s p a^{2} n^{2}$ rhoC rhoP

$+4 k s a^{4} k s p a^{2} n^{2} r h o P^{2}-12 k s a^{4} k s p a n^{2} r h o P^{2} j n p \_j n k s p a-12 k s a^{4} n^{4} r h o P^{2}$

$+2 k s a^{2} k s p a^{5}$ rhoC rhoP jnp_jnkspa $+2 k s a^{2} k s p a^{4} n^{2}$ rho $C^{2}-4 k s a^{2} k s p a^{4} n^{2}$ rhoC rhoP

$+24 k s a^{2} k s p a^{3} n^{2}$ rhoC rhoP jnp jnkspa $+24 k s a^{2} k s p a^{2} n^{4}$ rhoC rhoP

$-12 k s p a^{5} n^{2}$ rho $C^{2} j n p \_j n k p a-12 k s p a^{4} n^{4} r h o C^{2}-4 k s a^{4} k s p a^{2} n r h o P^{2}$

$-8 k s a^{4} k s p a n$ rhoP $P^{2}$ jnp jnkspa $+12 k s a^{4} n^{3} r h o P^{2}+4 k s a^{2} k s p a^{4} n$ rhoC rhoP

$+16 \mathrm{ksa}^{2} \mathrm{kspa} \mathrm{a}^{3} \mathrm{n}$ rhoC rhoP jnp jnkspa $-24 k s a^{2} k s p a^{2} n^{3}$ rhoC rhoP

$-8 k s p a^{5} n$ rho $C^{2}$ jnp jnkspa $+12 k s p a^{4} n^{3} r h o C^{2}-2 k s a^{4} k s p a^{2} r h o P^{2}+8 k s a^{4} n^{2} r h o P^{2}$

$+2 k s a^{2} k s p a^{4}$ rhoC rhoP $-16 k s a^{2} k s p a^{2} n^{2}$ rhoC rhoP $\left.+8 k s p a^{4} n^{2} r h o C^{2}\right) /($

-4 ksa $a^{5}$ spa ${ }^{4}$ rhoP $P^{2}$ hnp_hnksajnp_jnkspa

$+8 \mathrm{ksa}^{3} \mathrm{kspa}^{3} \mathrm{n}^{4}$ rhoCrhoP hnp_hnksajnp_jnkspa

$-4 k s a k s p a^{5} n^{4} r h o C^{2} h n p \_h n k s a j n p \_j n k s p a+k s a^{5} k s p a^{4} n$ rhoC rhoP hnp_hnksa

$+k s a^{5} k s p a^{4} n$ rhoP $P^{2} h n p \_h n k s a+2 k s a^{5} k_{s p a} a^{3} n$ rhoC rhoP hnp_hnksajnp_jnkspa

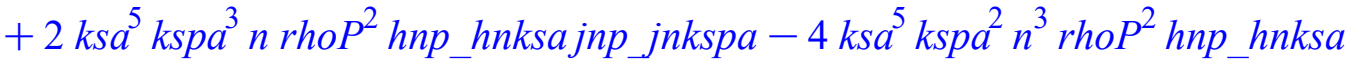

$-8 k s a^{5} k s p a n^{3}$ rhoP $P^{2} h n p \_h n k s a j n p \_j n k s p a+4 k s a^{5} n^{5} r h o P^{2}$ hnp_hnksa

- ksa $a^{4}$ spa $a^{5}$ rho $C^{2}$ jnp_jnkspa - ksa $a^{4}$ spa $a^{5}$ n rhoC rhoP jnp_jnkspa

$-4 k s a^{4} k s p a^{3} n^{3}$ rhoC rhoP jnp_jnkspa - $4 k s a^{4} k s p a n^{5}$ rho $P^{2} j n p \_j n k s p a$

$-2 \mathrm{ksa}^{3} \mathrm{kspa}^{5} \mathrm{n}$ rho $\mathrm{C}^{2}$ hnp_hnksajnp_jnkspa

$-2 \mathrm{ksa}^{3} \mathrm{kspa} \mathrm{a}^{5} \mathrm{n}$ rhoC rhoP hnp_hnksajnp_jnkspa $+4 \mathrm{ksa} a^{3} \mathrm{kspa} \mathrm{a}^{4} n^{3}$ rhoC rhoP hnp_hnksa

$+16 \mathrm{ksa}^{3} \mathrm{kspa}^{3} \mathrm{n}^{3}$ rhoC rhoP hnp_hnksajnp_jnkspa $-8 \mathrm{ksa} a^{3} \mathrm{kspa}^{2} n^{5}$ rhoC rhoP hnp_hnksa

$+4 k s a^{2} k s p a^{5} n^{3}$ rho $C^{2} j n p \_j n k s p a+8 k s a^{2} k s p a^{3} n^{5}$ rhoC rhoP jnp_jnkspa

$-8 k s a k s p a^{5} n^{3} r h o C^{2} h n p \_h n k s a j n p \_j n k s p a+4 k s a k s p a^{4} n^{5} r h o C^{2} h n p \_h n k s a$

$-4 k s p a^{5} n^{5} r h o C^{2} j n p \_j n k s p a+k s a^{5} k s p a^{4} r h o P^{2} h n p \_h n k s a$

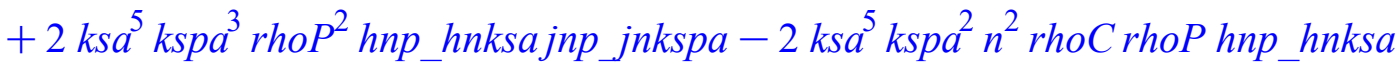

$-4 k s a^{5} k s p a^{2} n^{2} r h o P^{2} h n p \_h n k s a+4 k s a^{5} k s p a n^{2} r h o P^{2} h n p \_h n k s a j n p \_j n k s p a$ 
$+8 k s a^{5} n^{4} r h o P^{2} h n p \_h n k s a-k s a^{4} k s p a^{5} r h o C r h o P j n p \_j n k s p a+k s a^{4} k s p a^{4} n^{2} r h o C^{2}$

$-2 k s a^{4} k s p a^{4} n^{2} r h o C r h o P+k s a^{4} k s p a^{4} n^{2} r h o P^{2}-8 k s a^{4} k s p a^{3} n^{2}$ rhoC rhoP jnp jnkspa

$+2 k s a^{4} k s p a^{3} n^{2} r h o P^{2} j n p j n k s p a+4 k s a^{4} k s p a^{2} n^{4} r h o C r h o P-4 k s a^{4} k s p a^{2} n^{4} r h o P^{2}$

$-12 k_{s a} a^{4} k s p a n^{4} r h o P^{2} j n p j n k s p a+4 k s a^{4} n^{6} r h o P^{2}$

$-2 k_{s a}^{3} k s p a^{5}$ rhoC rhoP hnp_hnksajnp_jnkspa $+2 k_{s a} a^{3} k s p a^{4} n^{2} r h o C^{2} h n p \_h n k s a$

$+4 \mathrm{ksa}^{3} \mathrm{kspa} \mathrm{a}^{4} \mathrm{n}^{2}$ rhoC rhoP hnp_hnksa $-8 \mathrm{ksa}^{3} \mathrm{kspa}^{3} \mathrm{n}^{2}$ rhoC rhoP hnp_hnksajnp_jnkspa

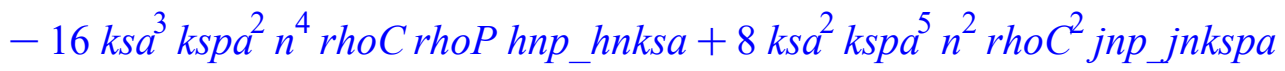

$-2 k s a^{2} k s p a^{5} n^{2}$ rhoC rhoP jnp jnkspa $-4 k s a^{2} k s p a^{4} n^{4} r h o C^{2}+4 k s a^{2} k s p a^{4} n^{4}$ rhoC rhoP

$+24 k s a^{2} k s p a^{3} n^{4}$ rhoC rhoP jnp jnkspa $-8 k s a^{2} k s p a^{2} n^{6}$ rhoC rhoP

+4 ksa kspa $a^{5} n^{2} r h o C^{2} h n p \_h n k s a j n p \_j n k s p a+8 k s a k s p a^{4} n^{4} r h o C^{2} h n p \_h n k s a$

$-12 k s p a^{5} n^{4} r h o C^{2} j n p \_j n k s p a+4 k s p a^{4} n^{6} r h o C^{2}+2 k s a^{5} k s p a^{2} n r h o P^{2} h n p \_h n k s a$

$+8 k s a^{5} k s p a n$ rho $P^{2} h n p \_h n k s a j n p$ jnkspa $-4 k s a^{5} n^{3} r h o P^{2} h n p \_h n k s a$

$-2 k_{s a}^{4} k s p a^{4} n$ rhoC rhoP $+2 k_{s a} a^{4} k s p a^{4} n r h o P^{2}-2 k s a^{4} k s p a^{3} n$ rhoC rhoP jnp jnkspa

$+4 k s a^{4} k s p a^{3} n r h o P^{2} j n p j n k s p a+8 k s a^{4} k s p a^{2} n^{3} r h o C r h o P-8 k s a^{4} k s p a^{2} n^{3} r h o P^{2}$

$-4 k s a^{4} k s p a n^{3} r h o P^{2} j n p j n k s p a+12 k s a^{4} n^{5} r h o P^{2}$

$-2 \mathrm{ksa}^{3} \mathrm{kspa}^{4} \mathrm{n}$ rhoC rhoP hnp_hnksa $-16 \mathrm{ksa}^{3} \mathrm{kspa}^{3} \mathrm{n}$ rhoC rhoP hnp_hnksa jnp_jnkspa

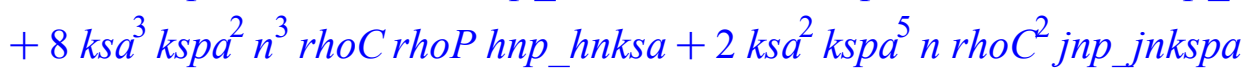

$-4 k s a^{2} k s p a^{5} n$ rhoC rhoP jnp jnkspa $-8 k s a^{2} k s p a^{4} n^{3} r h o C^{2}+8 k s a^{2} k s p a^{4} n^{3}$ rhoC rhoP

$+8 \mathrm{ksa}^{2} k s p a^{3} n^{3}$ rhoC rhoP jnp jnkspa $-24 k s a^{2} k s p a^{2} n^{5}$ rhoC rhoP

+8 ksakspa $n$ rho $C^{2}$ hnp_hnksajnp_jnkspa -4 ksakspa $n^{3}$ rhoC ${ }^{2}$ hnp_hnksa

$-4 k s p a^{5} n^{3} r h o C^{2} j n p$ jnkspa $+12 k s p a^{4} n^{5} r h o C^{2}+2 k s a^{5} k s p a^{2} r h o P^{2} h n p \_h n k s a$

$-8 k s a^{5} n^{2} r h o P^{2} h n p \_h n k s a-k s a^{4} k s p a^{4} r h o C r h o P+k s a^{4} k s p a^{4} r h o P^{2}$

$+2 \mathrm{ksa}^{4} \mathrm{kspa} \mathrm{a}^{3} \mathrm{rhoP^{2 }}$ jnp jnkspa $+2 \mathrm{ksa}^{4} \mathrm{kspa}^{2} n^{2} \mathrm{rhoC} r h o P-2 k s a^{4} k s p a^{2} n^{2} r h o P^{2}$

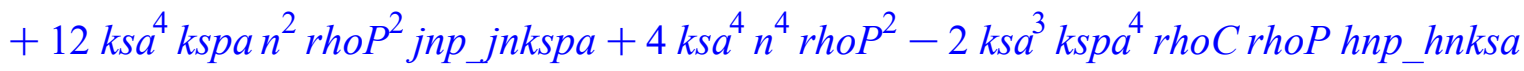

$+16 \mathrm{ksa}^{3} \mathrm{kspa}^{2} \mathrm{n}^{2}$ rhoC rhoP hnp_hnksa $-2 \mathrm{ksa}^{2} \mathrm{kspa} \mathrm{a}^{5}$ rhoC rhoP jnp jnkspa

$-2 k s a^{2} k s p a^{4} n^{2} r h o C^{2}+2 k s a^{2} k s p a^{4} n^{2}$ rhoC rhoP

$-24 k s a^{2} k s p a^{3} n^{2}$ rhoC rhoP jnp jnkspa $-8 k_{s} a^{2} k s p a^{2} n^{4}$ rhoC rhoP

$-8 k s a k s p a^{4} n^{2} r h o C^{2} h n p \_h n k s a+12 k s p a^{5} n^{2} r h o C^{2} j n p \_j n k s p a+4 k s p a^{4} n^{4} r h o C^{2}$

$+4 k s a^{4} k s p a^{2} n r h o P^{2}+8 k s a^{4} k s p a n r h o P^{2} j n p \_j n k s p a-12 k s a^{4} n^{3} r h o P^{2}$

$-4 \mathrm{ksa}^{2} \mathrm{kspa}^{4} \mathrm{n}$ rho C rhoP $-16 \mathrm{ksa}^{2} \mathrm{kspa} \mathrm{a}^{3} \mathrm{n}$ rhoC rhoP jnp jnkspa

$+24 k s a^{2} k s p a^{2} n^{3} r h o C r h o P+8 k s p a^{5} n r h o C^{2} j n p$ jnkspa $-12 k s p a^{4} n^{3} r h o C^{2}$

$+2 k s a^{4} k s p a^{2} r h o P^{2}-8 k s a^{4} n^{2} r h o P^{2}-2 k s a^{2} k s p a^{4}$ rhoC rhoP

$\left.+16 k s a^{2} k s p a^{2} n^{2} r h o C r h o P-8 k s p a^{4} n^{2} r h o C^{2}\right)$

$>$ numTnCS_: $=4 \mathrm{ksa}^{4} \mathrm{kspa}^{3} n^{2}$ rhoC rhoP jnp_jnkspa $+8 \mathrm{ksa}^{4} \mathrm{kspan} \mathrm{n}^{4} \mathrm{rhoP^{2 }}$ jnp jnkspa

$-4 k s a^{2} k s p a^{5} n^{2} r h o C^{2} j n p \_j n k s p a-16 k_{s} a^{2} k_{s p a} n^{3}{ }^{4}$ rhoC rhoP jnp jnkspa

$+8 \mathrm{kspa}^{5} n^{4} \mathrm{rhoC}^{2}$ jnp jnkspa $+2 \mathrm{ksa}^{4} \mathrm{kspa}^{4} \mathrm{n}$ rhoC rhoP $-2 \mathrm{ksa}^{4} \mathrm{kspa}^{4} \mathrm{n} r \mathrm{rhoP} \mathrm{P}^{2}$ 
$+2 k_{s a}^{4} k s p a^{3} n$ rhoC rhoP jnp_jnkspa $-4 k s a^{4} k s p a^{3} n$ rhoP $P^{2}$ jnp_jnkspa

$-4 k s a^{4} k s p a^{2} n^{3} r h o C r h o P+8 k s a^{4} k s p a^{2} n^{3} r h o P^{2}+12 k s a^{4} k s p a n^{3} r h o P^{2} j n p j n k s p a$

$-8 k s a^{4} n^{5} r h o P^{2}-2 k s a^{2} k s p a^{5} n r h o C^{2} j n p$ jnkspa $+4 k s a^{2} k s p a^{5} n$ rhoC rhoP jnp jnkspa

$+4 k s a^{2} k s p a^{4} n^{3} r h o C^{2}-8 k s a^{2} k s p a^{4} n^{3}$ rhoC rhoP

$-24 \mathrm{ksa}^{2} \mathrm{kspa} \mathrm{a}^{3} \mathrm{n}^{3}$ rhoC rhoP jnp jnkspa $+16 \mathrm{ksa}^{2} \mathrm{kspa}^{2} \mathrm{n}^{5}$ rhoC rhoP

$+12 k s p a^{5} n^{3} r h o C^{2}$ jnp jnkspa $-8 k s p a^{4} n^{5} r h o C^{2}+k s a^{4} k s p a^{4} r h o C r h o P$

$-k s a^{4} k s p a^{4} r h o P^{2}-2 k s a^{4} k s p a^{3} r h o P^{2} j n p$ jnkspa $-2 k s a^{4} k s p a^{2} n^{2}$ rhoC rhoP

$+4 k s a^{4} k s p a^{2} n^{2} r h o P^{2}-12 k s a^{4} k s p a n^{2} r h o P^{2} j n p$ jnkspa $-12 k s a^{4} n^{4} r h o P^{2}$

$+2 k s a^{2} k s p a^{5}$ rhoC rhoP jnp jnkspa $+2 k s a^{2} k s p a^{4} n^{2} r h o C^{2}-4 k s a^{2} k s p a^{4} n^{2}$ rhoC rhoP

$+24 k s a^{2} k s p a^{3} n^{2}$ rhoC rhoP jnp jnkspa $+24 k s a^{2} k s p a^{2} n^{4}$ rhoCrhoP

$-12 k s p a^{5} n^{2} r h o C^{2}$ jnp jnkspa $-12 k s p a^{4} n^{4} r h o C^{2}-4 k s a^{4} k s p a^{2} n r h o P^{2}$

$-8 \mathrm{ksa}^{4} \mathrm{kspa} n \mathrm{rhoP^{2 }}$ jnp_jnkspa $+12 \mathrm{ksa}^{4} n^{3} \mathrm{rhoP}^{2}+4 \mathrm{ksa}^{2} \mathrm{kspa} a^{4} \mathrm{n}$ rhoC rhoP

$+16 \mathrm{ksa}^{2} k s p a^{3} n$ rhoC rhoP jnp jnkspa $-24 k s a^{2} k s p a^{2} n^{3}$ rhoC rhoP

$-8 k s p a^{5} n r h o C^{2}$ jnp jnkspa $+12 k s p a^{4} n^{3} r h o C^{2}-2 k s a^{4} k s p a^{2} r h o P^{2}+8 k s a^{4} n^{2} r h o P^{2}$

$+2 k s a^{2} k s p a^{4}$ rhoC rhoP $-16 k s a^{2} k s p a^{2} n^{2}$ rhoC rhoP $+8 k s p a^{4} n^{2} r h o C^{2}:$

$>\operatorname{denTnCS}:=-4 \mathrm{ksa}^{5} \mathrm{kspan} \mathrm{n}^{4} \mathrm{rhoP^{2 }}$ hnp_hnksajnp_jnkspa

$+8 \mathrm{ksa}^{3} \mathrm{kspa}^{3} \mathrm{n}^{4}$ rhoC rhoP hnp_hnksajnp_jnkspa

-4 ksa kspa ${ }^{5} n^{4}$ rho $C^{2}$ hnp_hnksajnp_jnkspa + ksa $a^{5}$ kspa ${ }^{4}$ n rhoC rhoP hnp_hnksa

$+k s a^{5} k s p a^{4} n$ rhoP $P^{2} h n p \_h n k s a+2 k_{s} a^{5} k s p a^{3} n$ rhoC rhoP hnp_hnksajnp_jnkspa

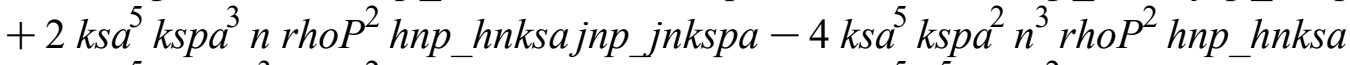

$-8 k s a^{5} k s p a n^{3}$ rhoP $P^{2}$ hnp_hnksajnp_jnkspa $+4 k s a^{5} n^{5} r h o P^{2}$ hnp_hnksa

$-k s a^{4} k s p a^{5} n$ rho $C^{2}$ jnp jnkspa - ksa kspa $n$ rhoC rhoP jnp jnkspa

$-4 k s a^{4} k s p a^{3} n^{3}$ rhoC rhoP jnp jnkspa $-4 k s a^{4} k s p a n^{5}$ rhoP $P^{2}$ jnp jnkspa

$-2 \mathrm{ksa}^{3} \mathrm{kspa} \mathrm{a}^{5} \mathrm{n} r h o C^{2}$ hnp_hnksajnp_jnkspa

$-2 k_{s a} a^{3} k s p a^{5} n$ rhoC rhoP hnp_hnksajnp_jnkspa $+4 k_{s a}^{3} k_{s p a} n^{3}$ rhoCrhoP hnp_hnksa

$+16 \mathrm{ksa}^{3} \mathrm{kspa}^{3} n^{3}$ rhoCrhoP hnp_hnksajnp_jnkspa $-8 \mathrm{ksa}^{3} \mathrm{kspa}^{2} n^{5}$ rhoC rhoP hnp_hnksa

$+4 \mathrm{ksa}^{2} k s p a^{5} n^{3} r h o C^{2} j n p$ jnkspa $+8 \mathrm{ksa}^{2} \mathrm{kspa}^{3} n^{5}$ rhoC rhoP jnp jnkspa

$-8 k s a k s p a^{5} n^{3}$ rho $C^{2} h n p \_h n k s a j n p \_j n k s p a+4 k s a k s p a^{4} n^{5}$ rho $C^{2} h n p \_h n k s a$

$-4 k s p a^{5} n^{5} r h o C^{2}$ jnp_jnkspa $+k s a^{5} k s p a^{4} r h o P^{2}$ hnp_hnksa

$+2 k s a^{5} k s p a^{3}$ rhoP $P^{2}$ hnp hnksa jnp jnkspa $-2 k s a^{5} k s p a^{2} n^{2}$ rhoC rhoP hnp hnksa

$-4 k s a^{5} k s p a^{2} n^{2} r h o P^{2} h n p \_h n k s a+4 k s a^{5} k s p a n^{2} r h o P^{2} h n p \_h n k s a j n p \_j n k s p a$

$+8 k s a^{5} n^{4} r h o P^{2} h n p \_h n k s a-k s a^{4} k s p a^{5}$ rhoC rhoP jnp_jnkspa $+k s a^{4} k s p a^{4} n^{2} r h o C^{2}$

$-2 k s a^{4} k s p a^{4} n^{2} r h o C r h o P+k s a^{4} k s p a^{4} n^{2} r h o P^{2}-8 k s a^{4} k s p a^{3} n^{2} r h o C r h o P$ jnp jnkspa

$+2 k s a^{4} k s p a^{3} n^{2} r h o P^{2} j n p j j n k s p a+4 k s a^{4} k s p a^{2} n^{4}$ rhoC rhoP $-4 k s a^{4} k s p a^{2} n^{4} r h o P^{2}$

$-12 k_{s a} a^{4} k s p a n^{4} r h o P^{2}$ jnp jnkspa $+4 k s a^{4} n^{6} r h o P^{2}$

$-2 \mathrm{ksa}^{3} \mathrm{kspa} a^{5}$ rhoC rhoP hnp_hnksajnp jnkspa $+2 \mathrm{ksa}^{3} \mathrm{kspa} \mathrm{a}^{4} \mathrm{n}^{2}$ rho $\mathrm{C}^{2}$ hnp_hnksa

$+4 \mathrm{ksa}^{3} \mathrm{kspa} \mathrm{a}^{4} \mathrm{n}^{2}$ rhoC rhoP hnp_hnksa $-8 \mathrm{ksa}^{3} \mathrm{kspa} \mathrm{a}^{3} \mathrm{n}^{2}$ rhoC rhoP hnp_hnksajnp_jnkspa

$-16 \mathrm{ksa}^{3} \mathrm{kspa}^{2} \mathrm{n}^{4}$ rhoCrhoP hnp_hnksa $+8 \mathrm{ksa}^{2} \mathrm{kspa}^{5} n^{2}$ rho $\mathrm{C}^{2}$ jnp_jnkspa

$-2 k s a^{2} k s p a^{5} n^{2}$ rhoC rhoP jnp jnkspa $-4 k s a^{2} k s p a^{4} n^{4} r h o C^{2}+4 k s a^{2} k s p a^{4} n^{4}$ rhoC rhoP

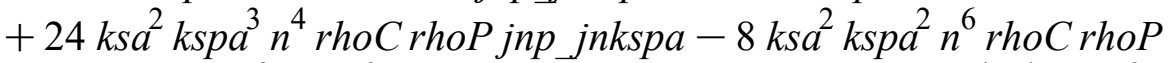

$+4 k s a k s p a^{5} n^{2} r h o C^{2}$ hnp_hnksajnp_jnkspa $+8 k s a k s p a^{4} n^{4} r h o C^{2}$ hnp_hnksa

$-12 k s p a^{5} n^{4} r h o C^{2} j n p \_j n k s p a+4 k s p a^{4} n^{6} r h o C^{2}+2 k s a^{5} k s p a^{2} n r h o P^{2} h n p \_h n k s a$ 
$+8 k s a^{5} k s p a n$ rhoP $P^{2}$ hnp_hnksajnp jnkspa $-4 k^{2} a^{5} n^{3} r h o P^{2} h n p \_h n k s a$

$-2 k_{s a} a^{4} k s p a^{4} n$ rhoC rhoP $+2 k s a^{4} k s p a^{4} n r h o P^{2}-2 k s a^{4} k s p a^{3} n$ rhoCrhoP jnp jnkspa

$+4 k s a^{4} k s p a^{3} n r h o P^{2} j n p$ jnkspa $+8 k s a^{4} k s p a^{2} n^{3}$ rhoC rhoP $-8 k s a^{4} k s p a^{2} n^{3} r h o P^{2}$

$-4 k s a^{4} k s p a n^{3}$ rhoP $P^{2}$ jnp jnkspa $+12 k s a^{4} n^{5} r h o P^{2}-2 k s a^{3} k s p a^{4} n$ rhoC rhoP hnp hnksa

$-16 \mathrm{ksa}^{3} \mathrm{kspa}^{3} \mathrm{n}$ rhoCrhoP hnp_hnksa jnp_jnkspa $+8 \mathrm{ksa}^{3} \mathrm{kspa}^{2} \mathrm{n}^{3}$ rhoCrhoP hnp_hnksa

$+2 \mathrm{ksa}^{2} \mathrm{kspa} \mathrm{a}^{5} \mathrm{n} r h o \mathrm{C}^{2}$ jnp jnkspa $-4 \mathrm{ksa}^{2} \mathrm{kspa}^{5}$ n rhoC rhoP jnp jnkspa

$-8 k s a^{2} k s p a^{4} n^{3} r h o C^{2}+8 k s a^{2} k s p a^{4} n^{3} r h o C r h o P+8 k s a^{2} k s p a^{3} n^{3}$ rhoC rhoP jnp jnkspa

$-24 \mathrm{ksa}^{2} \mathrm{kspa}^{2} n^{5}$ rhoCrhoP $+8 \mathrm{ksa} k s p a^{5} n$ rho $\mathrm{C}^{2}$ hnp_hnksajnp_jnkspa

$-4 k s a k s p a^{4} n^{3} r h o C^{2} h n p \_h n k s a-4 k s p a^{5} n^{3} r h o C^{2} j n p j$ jnkspa $+12 k s p a^{4} n^{5} r h o C^{2}$

$+2 k s a^{5} k s p a^{2} r h o P^{2} h n p \_h n k s a-8 k s a^{5} n^{2} r h o P^{2} h n p \_h n k s a-k s a^{4} k s p a^{4}$ rhoC rhoP

$+k s a^{4} k s p a^{4} r h o P^{2}+2 k s a^{4} k s p a^{3} r h o P^{2} j n p j n k s p a+2 k s a^{4} k s p a^{2} n^{2}$ rhoC rhoP

$-2 k s a^{4} k s p a^{2} n^{2} r h o P^{2}+12 k s a^{4} k s p a n^{2} r h o P^{2} j n p j$ jnkspa $+4 k s a^{4} n^{4} r h o P^{2}$

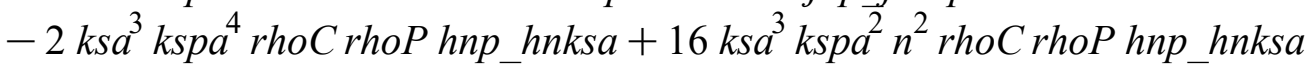

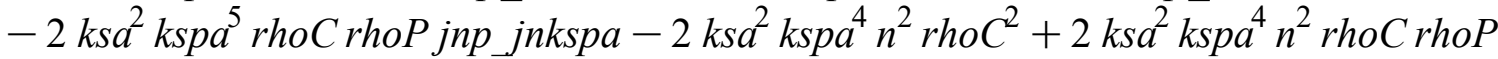

$-24 k s a^{2} k s p a^{3} n^{2}$ rhoCrhoP jnp jnkspa $-8 k s a^{2} k s p a^{2} n^{4}$ rhoC rhoP

$-8 k s a k s p a^{4} n^{2} r h o C^{2} h n p \_h n k s a+12 k s p a^{5} n^{2} r h o C^{2} j n p j n k s p a+4 k s p a^{4} n^{4} r h o C^{2}$

$+4 k s a^{4} k s p a^{2} n r h o P^{2}+8 k s a^{4} k s p a n r h o P^{2} j n p j$ jnkspa $-12 k s a^{4} n^{3} r h o P^{2}$

$-4 \mathrm{ksa}^{2} \mathrm{kspa}^{4} \mathrm{n}$ rhoC rhoP $-16 \mathrm{ksa}^{2} \mathrm{kspa} \mathrm{a}^{3} \mathrm{n}$ rhoC rhoP jnp jnkspa

$+24 \mathrm{ksa}^{2} \mathrm{kspa}^{2} \mathrm{n}^{3}$ rhoCrhoP $+8 \mathrm{kspa} n \mathrm{a}^{5} \mathrm{rhoC^{2 }}$ jnp jnkspa $-12 k s p a^{4} n^{3} r h o C^{2}$

$+2 k s a^{4} k s p a^{2} r h o P^{2}-8 k s a^{4} n^{2} r h o P^{2}-2 k s a^{2} k s p a^{4}$ rhoC rhoP

$+16 k s a^{2} k s p a^{2} n^{2} r h o C r h o P-8 k s p a^{4} n^{2} r h o C^{2}:$

$>\operatorname{denTnCS} \_$- denTnCC_

\#check that the denominators are the same for the coefficients - this saves having to derive the simplified form of the denominator

$>$ TnSCLeadkca $:=(\operatorname{series}($ 'leadterm' $(($ TnSC_ $)), k c a, 1))$

TnSCLeadkca_: $=-\left(n(n+1) k s a\left(2 \mathrm{ksa}^{4} \mathrm{kspa} \mathrm{3}^{3} \mathrm{n}\right.\right.$ rhoC rhoP hnp_hnksa jnp_jnkspa

$-2 k_{s a}^{4} k s p a^{3}$ n rhoC rhoP jnp jnksa jnp _jnkspa

$+4 k s a^{4} k s p a n^{3} r h o P^{2} h n p \_h n k s a j n p \_j n k s p a-4 k s a^{4} k s p a n^{3} r h o P^{2}$ jnp jnksa jnp_jnkspa

$-2 k s a^{2} k s p a^{5} n r h o C^{2} h n p \_h n k s a j n p \_j n k s p a+2 k s a^{2} k s p a^{5} n r h o C^{2} j n p$ jnksa jnp jnkspa

$-8 \mathrm{ksa}^{2} \mathrm{kspa}^{3} n^{3}$ rhoCrhoP hnp_hnksajnp_jnkspa

$+8 \mathrm{ksa}^{2} k s p a^{3} n^{3}$ rhoC rhoP jnp_jnksa jnp_jnkspa $+4 k s p a^{5} n^{3} r h o C^{2} h n p \_h n k s a j n p \_j n k s p a$

$-4 k s p a^{5} n^{3} r h o C^{2}$ jnp_jnksa jnp_jnkspa $+k s a^{4} k s p a^{4}$ rhoC rhoP hnp_hnksa

- ksa $a^{4}$ sspa ${ }^{4}$ rhoCrhoP jnp_jnksa - ksa $a^{4} k s p a^{4}$ rho $P^{2}$ hnp_hnksa

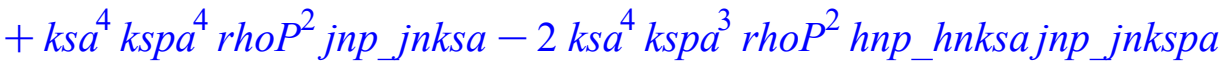

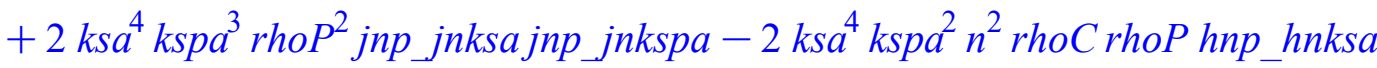

$+2 k s a^{4} k s p a^{2} n^{2}$ rhoC rhoP jnp_jnksa $+4 k s a^{4} k s p a^{2} n^{2} r h o P^{2} h n p \_h n k s a$

$-4 k s a^{4} k s p a^{2} n^{2} r h o P^{2} j n p \_j n k s a+4 k s a^{4} k s p a n^{2} r h o P^{2} h n p \_h n k s a j n p \_j n k s p a$

$-4 k s a^{4} k s p a n^{2} r h o P^{2} j n p$ jnksajnp_jnkspa $-4 k s a^{4} n^{4} r h o P^{2} h n p \_h n k s a$

$+4 k s a^{4} n^{4} r h o P^{2} j n p \_j n k s a+2 k s a^{2} k s p a^{5} r h o C r h o P$ hnp_hnksajnp_jnkspa 
$-2 k s a^{2} k s p a^{5}$ rhoC rhoP jnp_jnksa jnp_jnkspa $+2 k s a^{2} k s p a^{4} n^{2} r h o C^{2} h n p \_h n k s a$

$-2 k s a^{2} k s p a^{4} n^{2}$ rho $C^{2} j n p \_j n k s a-4 k s a^{2} k s p a^{4} n^{2}$ rhoCrhoP hnp_hnksa

$+4 k s a^{2} k s p a^{4} n^{2}$ rhoC rhoP jnp_jnksa $-8 k s a^{2} k s p a^{3} n^{2}$ rhoC rhoP hnp_hnksajnp_jnkspa

$+8 k s a^{2} k s p a^{3} n^{2}$ rhoC rhoP jnp_jnksajnp_jnkspa $+8 k s a^{2} k s p a^{2} n^{4}$ rhoC rhoP hnp_hnksa

$-8 \mathrm{ksa}^{2} \mathrm{kspa}^{2} n^{4}$ rhoC rhoP jnp_jnksa $+4 k s p a^{5} n^{2}$ rho $C^{2}$ hnp_hnksajnp_jnkspa

$-4 k s p a^{5} n^{2} r h o C^{2} j n p \_j n k s a j n p \_j n k s p a-4 k s p a^{4} n^{4} r h o C^{2} h n p \_h n k s a$

$+4 k s p a^{4} n^{4} r h o C^{2} j n p \_j n k s a-8 k s a^{4} k s p a n$ rhoP $P^{2}$ hnp_hnksajnp_jnkspa

$+8 k s a^{4} k s p a n$ rhoP $P^{2}$ jnp_jnksajnp_jnkspa $-4 k s a^{4} n^{3}$ rhoP $P^{2} h n p \_h n k s a$

$+4 k s a^{4} n^{3} r h o P^{2} j n p \_j n k s a+16 k s s^{2} k s p a^{3} n$ rhoC rhoP hnp_hnksajnp_jnkspa

$-16 \mathrm{ksa}^{2} \mathrm{kspa}^{3} \mathrm{n}$ rhoC rhoP jnp_jnksajnp_jnkspa $+8 \mathrm{ksa}^{2} k s p a^{2} n^{3}$ rhoC rhoP hnp_hnksa

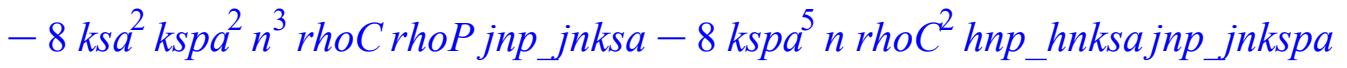

$+8 k s p a^{5} n$ rho $C^{2} j n p \_j n k s a j n p \_j n k s p a-4 k s p a^{4} n^{3}$ rho $C^{2} h n p \_h n k s a$

$+4 k s p a^{4} n^{3}$ rho $C^{2} j n p \_j n k s a-2 k s a^{4} k s p a^{2} r h o P^{2} h n p \_h n k s a$

$+2 k s a^{4} k s p a^{2} r h o P^{2} j n p \_j n k s a+8 k s a^{4} n^{2} r h o P^{2} h n p \_h n k s a-8 k s a^{4} n^{2} r h o P^{2} j n p \_j n k s a$

$+2 \mathrm{ksa}^{2} \mathrm{kspa} \mathrm{a}^{4}$ rhoC rhoP hnp_hnksa $-2 \mathrm{ksa}^{2} \mathrm{kspa} \mathrm{a}^{4}$ rhoC rhoP jnp_jnksa

$-16 k s a^{2} k s p a^{2} n^{2}$ rhoC rhoP hnp_hnksa $+16 k s a^{2} k s p a^{2} n^{2}$ rhoC rhoP jnp_jnksa

$\left.\left.+8 k s p a^{4} n^{2} r h o C^{2} h n p \_h n k s a-8 k s p a^{4} n^{2} r h o C^{2} j n p \_j n k s a\right)\right) /($

-4 ksa $a^{5}$ kspan ${ }^{4}$ rhoP $P^{2}$ hnp_hnksajnp_jnkspa

$+8 \mathrm{ksa}^{3} \mathrm{kspa}^{3} \mathrm{n}^{4}$ rhoC rhoP hnp_hnksajnp_jnkspa

$-4 k s a k s p a^{5} n^{4}$ rho $C^{2} h n p \_h n k s a j n p \_j n k s p a+k s a^{5} k s p a^{4} n$ rhoC rhoP hnp_hnksa

$+k s a^{5} k s p a^{4} n$ rhoP $P^{2}$ hnp_hnksa $+2 k s a^{5} k_{s p a}{ }^{3} n$ rhoC rhoP hnp_hnksajnp_jnkspa

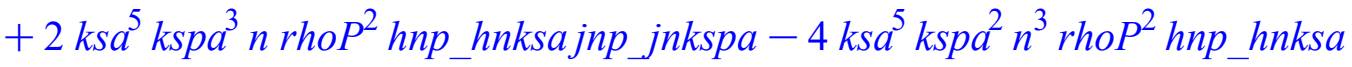

$-8 k s a^{5} k s p a n^{3} r h o P^{2} h n p \_h n k s a j n p \_j n k s p a+4 k s a^{5} n^{5} r h o P^{2}$ hnp_hnksa

$-k s a^{4} k s p a^{5} n$ rho $C^{2} j n p \_j n k s p a-k s a^{4} k s p a^{5} n$ rhoC rhoP jnp_jnkspa

$-4 k s a^{4} k s p a^{3} n^{3}$ rhoC rhoP jnp_jnkspa $-4 k s a^{4} k s p a n^{5}$ rhoP ${ }^{2}{ }_{j n p} j n k s p a$

$-2 \mathrm{ksa}^{3} \mathrm{kspa}^{5} \mathrm{n}$ rho $\mathrm{C}^{2}$ hnp_hnksajnp_jnkspa

$-2 \mathrm{ksa}^{3} \mathrm{kspa}^{5}$ n rhoC rhoP hnp_hnksajnp_jnkspa $+4 \mathrm{ksa}^{3} \mathrm{kspa}^{4} \mathrm{n}^{3}$ rhoC rhoP hnp_hnksa

$+16 \mathrm{ksa}^{3} \mathrm{kspa}^{3} \mathrm{n}^{3}$ rhoC rhoP hnp_hnksajnp_jnkspa - $8 \mathrm{ksa} a^{3} \mathrm{kspa} a^{2} n^{5}$ rhoC rhoP hnp_hnksa

$+4 k s a^{2} k s p a^{5} n^{3} r h o C^{2} j n p \_j n k s p a+8 k s a^{2} k s p a^{3} n^{5}$ rhoC rhoP jnp_jnkspa

$-8 k s a k s p a^{5} n^{3} r h o C^{2} h n p \_h n k s a j n p \_j n k s p a+4 k s a k s p a^{4} n^{5} r h o C^{2} h n p \_h n k s a$

$-4 k s p a^{5} n^{5} r h o C^{2} j n p \_j n k s p a+k s a^{5} k s p a^{4} r h o P^{2} h n p \_h n k s a$

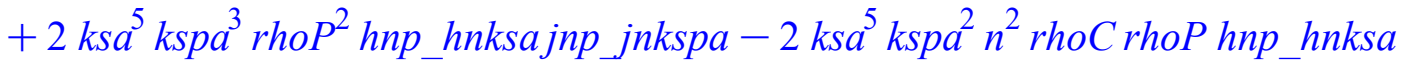

$-4 k s a^{5} k s p a^{2} n^{2} r h o P^{2} h n p \_h n k s a+4 k s a^{5} k s p a n^{2} r h o P^{2} h n p \_h n k s a j n p \_j n k s p a$

$+8 k s a^{5} n^{4}$ rhoP $P^{2}$ hnp_hnksa $-k s a^{4} k s p a^{5}$ rhoC rhoP jnp jnkspa $+k s a^{4} k s p a^{4} n^{2} r h o C^{2}$

$-2 k s a^{4} k s p a^{4} n^{2}$ rhoC rhoP $+k s a^{4} k s p a^{4} n^{2} r h o P^{2}-8 k s a^{4} k s p a^{3} n^{2}$ rhoC rhoP jnp jnkspa

$+2 k s a^{4} k s p a^{3} n^{2} r h o P^{2} j n p \_j n k s p a+4 k s a^{4} k s p a^{2} n^{4}$ rhoC rhoP $-4 k s a^{4} k s p a^{2} n^{4} r h o P^{2}$ 
$-12 k s a^{4} k s p a n^{4} r h o P^{2} j n p \_j n k s p a+4 k s a^{4} n^{6} r h o P^{2}$

$-2 \mathrm{ksa}^{3} \mathrm{kspa} \mathrm{a}^{5}$ rhoC rhoP hnp_hnksajnp_jnkspa $+2 \mathrm{ksa} a^{3} \mathrm{kspa} \mathrm{a}^{4} \mathrm{n}^{2} \mathrm{rhoC} \mathrm{C}^{2} \mathrm{hnp} \_$hnksa

$+4 \mathrm{ksa}^{3} \mathrm{kspa}^{4} \mathrm{n}^{2}$ rhoC rhoP hnp_hnksa $-8 \mathrm{ksa}^{3} \mathrm{kspa}^{3} \mathrm{n}^{2}$ rhoC rhoP hnp_hnksajnp_jnkspa

$-16 k s a^{3} k s p a^{2} n^{4}$ rhoC rhoP hnp_hnksa $+8 k s a^{2} k s p a^{5} n^{2} r h o C^{2} j n p \_j n k s p a$

$-2 k s a^{2} k s p a^{5} n^{2}$ rhoC rhoP jnp_jnkspa $-4 k s a^{2} k s p a^{4} n^{4} r h o C^{2}+4 k s a^{2} k s p a^{4} n^{4}$ rhoC rhoP

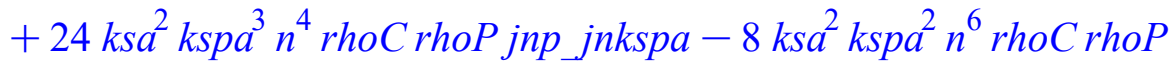

$+4 k s a k s p a^{5} n^{2} r h o C^{2} h n p \_h n k s a j n p \_j n k s p a+8 k s a k s p a^{4} n^{4} r h o C^{2} h n p \_h n k s a$

$-12 k s p a^{5} n^{4} r h o C^{2} j n p \_j n k s p a+4 k s p a^{4} n^{6} r h o C^{2}+2 k s a^{5} k s p a^{2} n r h o P^{2} h n p \_h n k s a$

$+8 k s a^{5}$ kspa $n$ rhoP $P^{2}$ hnp_hnksajnp_jnkspa $-4 k s a^{5} n^{3} r h o P^{2}$ hnp_hnksa

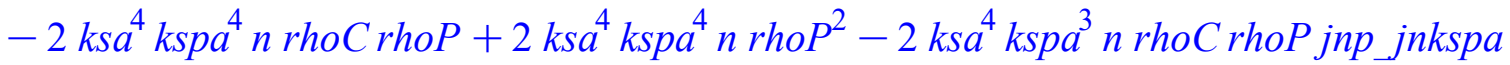

$+4 k s a^{4} k s p a^{3} n r h o P^{2} j n p \_j n k s p a+8 k s a^{4} k s p a^{2} n^{3}$ rhoC rhoP $-8 k s a^{4} k s p a^{2} n^{3} r h o P^{2}$

$-4 k s a^{4} k s p a n^{3} r h o P^{2} j n p \_j n k s p a+12 k s a^{4} n^{5} r h o P^{2}$

$-2 \mathrm{ksa}^{3} \mathrm{kspa}^{4} \mathrm{n}$ rhoC rhoP hnp_hnksa $-16 \mathrm{ksa}^{3} \mathrm{kspa}^{3} \mathrm{n}$ rhoC rhoP hnp_hnksa jnp_jnkspa

$+8 k s a^{3} k s p a^{2} n^{3}$ rhoC rhoP hnp_hnksa $+2{k n s a^{2}}_{k s p a}{ }^{5} n$ rho $C^{2}$ jnp jnkspa

$-4 k s a^{2} k s p a^{5} n$ rhoC rhoP jnp jnkspa $-8 k s a^{2} k s p a^{4} n^{3} r h o C^{2}+8 k s a^{2} k s p a^{4} n^{3}$ rhoC rhoP

$+8 k s a^{2} k s p a^{3} n^{3}$ rhoC rhoP jnp_jnkspa $-24 k s a^{2} k s p a^{2} n^{5}$ rhoC rhoP

$+8 k s a k s p a^{5} n$ rho $C^{2} h n p \_h n k s a j n p \_j n k s p a-4 k s a k s p a^{4} n^{3}$ rho $C^{2} h n p \_h n k s a$

$-4 k s p a^{5} n^{3} r h o C^{2} j n p \_j n k s p a+12 k s p a^{4} n^{5} r h o C^{2}+2 k s a^{5} k s p a^{2} r h o P^{2} h n p \_h n k s a$

$-8 k s a^{5} n^{2} r h o P^{2} h n p \_h n k s a-k s a^{4} k s p a^{4}$ rhoCrhoP $+k s a^{4} k s p a^{4} r h o P^{2}$

$+2 k s a^{4} k s p a^{3} r h o P^{2} j n p \_j n k s p a+2 k s a^{4} k s p a^{2} n^{2}$ rhoC rhoP $-2 k s a^{4} k s p a^{2} n^{2} r h o P^{2}$

$+12 \mathrm{ksa}^{4} \mathrm{kspan}^{2} \mathrm{rhoP}^{2}$ jnp_jnkspa $+4 \mathrm{ksa}^{4} \mathrm{n}^{4} \mathrm{rhoP}^{2}-2 \mathrm{ksa} \mathrm{k}^{3} \mathrm{kspa} \mathrm{a}^{4} \mathrm{rhoC}$ rhoP hnp_hnksa

$+16 \mathrm{ksa}^{3} \mathrm{kspa}^{2} \mathrm{n}^{2}$ rhoC rhoP hnp_hnksa $-2 \mathrm{ksa}^{2} \mathrm{kspa} \mathrm{a}^{5}$ rhoC rhoP jnp_jnkspa

$-2 k s a^{2} k s p a^{4} n^{2} r h o C^{2}+2 k s a^{2} k s p a^{4} n^{2}$ rhoC rhoP

$-24 k s a^{2} k s p a^{3} n^{2}$ rhoC rhoP jnp_jnkspa $-8 k s a^{2} k s p a^{2} n^{4}$ rhoC rhoP

$-8 k s a k s p a^{4} n^{2}$ rho $C^{2}$ hnp_hnksa $+12 k s p a^{5} n^{2} r h o C^{2} j n p \_j n k s p a+4 k s p a^{4} n^{4} r_{h o C^{2}}$

$+4 k s a^{4} k s p a^{2} n r h o P^{2}+8 k s a^{4} k s p a n r h o P^{2} j n p j n k s p a-12 k s a^{4} n^{3} r h o P^{2}$

$-4 \mathrm{ksa}^{2} \mathrm{kspa}^{4} \mathrm{n}$ rhoC rhoP - $16 \mathrm{ksa}^{2} \mathrm{kspa}^{3} \mathrm{n}$ rhoC rhoP jnp_jnkspa

$+24 k s a^{2} k s p a^{2} n^{3}$ rhoC rhoP $+8 k s p a^{5} n$ rho $C^{2} j n p \_j n k s p a-12 k s p a^{4} n^{3} r_{h o C^{2}}$

$+2 k s a^{4} k s p a^{2} r h o P^{2}-8 k s a^{4} n^{2} r h o P^{2}-2 k s a^{2} k s p a^{4}$ rhoC rhoP

$+16 k s a^{2} k s p a^{2} n^{2}$ rhoC rhoP $\left.-8 k s p a^{4} n^{2} r h o C^{2}\right)$

$>$ numTnSC_: $=-n(n+1) k s a\left(2 k s a^{4} k s p a^{3} n\right.$ rhoC rhoP hnp_hnksajnp_jnkspa

$-2 \mathrm{ksa}^{4} \mathrm{kspa}^{3} \mathrm{n}$ rhoC rhoP jnp_jnksa jnp_jnkspa

$+4 \mathrm{ksa}^{4} \mathrm{kspa}^{3} \mathrm{rhoP}^{2}$ hnp_hnksajnp_jnkspa $-4 \mathrm{ksa}^{4} \mathrm{kspa}^{3} \mathrm{rhoP}^{2}$ jnp_jnksa jnp_jnkspa

$-2 k s a^{2} k s p a^{5} n r h o C^{2}$ hnp_hnksajnp_jnkspa $+2 k s a^{2} k s p a^{5} n$ rho $C^{2}$ jnp_jnksa jnp_jnkspa

$-8 \mathrm{ksa}^{2} \mathrm{kspa}^{3} \mathrm{n}^{3}$ rhoC rhoP hnp_hnksajnp_jnkspa

$+8 k s a^{2} k s p a^{3} n^{3}$ rhoCrhoP jnp_jnksajnp_jnkspa $+4 k s p a^{5} n^{3}$ rho $C^{2}$ hnp_hnksajnp_jnkspa

$-4 k s p a^{5} n^{3}$ rho ${ }^{2}$ jnp jnksajnp jnkspa $+k s a^{4} k s p a^{4}$ rhoC rhoP hnp hnksa 
- ksa $a^{4}$ spa $a^{4}$ rhoC rhoP jnp_jnksa - ksa ${ }^{4} k s p a^{4}$ rhoP $P^{2}$ hnp_hnksa

$+k s a^{4} k s p a^{4} r h o P^{2} j n p \_j n k s a-2 k s a^{4} k s p a^{3}$ rhoP $P^{2} h n p \_h n k s a j n p \_j n k s p a$

$+2 \mathrm{ksa}^{4} \mathrm{kspa} \mathrm{a}^{3} \mathrm{rhoP} \mathrm{P}^{2}$ jnp_jnksa jnp_jnkspa $-2 \mathrm{ksa}^{4} \mathrm{kspa} \mathrm{a}^{2} \mathrm{n}^{2}$ rhoC rhoP hnp_hnksa

$+2 k s a^{4} k s p a^{2} n^{2}$ rhoC rhoP jnp_jnksa $+4 k s a^{4} k s p a^{2} n^{2}$ rhoP $P^{2}$ hnp_hnksa

$-4 k s a^{4} k s p a^{2} n^{2}$ rhoP $P^{2}$ jnp_jnksa $+4 k s a^{4} k s p a n^{2}$ rhoP $P^{2}$ hnp_hnksajnp_jnkspa

$-4 k s a^{4} k s p a n^{2}$ rhoP $P^{2}$ jnp_jnksa jnp_jnkspa $-4 k s a^{4} n^{4}$ rho $P^{2}$ hnp_hnksa

$+4 \mathrm{ksa}^{4} \mathrm{n}^{4} \mathrm{rhoP}^{2}$ jnp_jnksa $+2 \mathrm{ksa}^{2} \mathrm{kspa}^{5}$ rhoC rhoP hnp_hnksajnp_jnkspa

$-2 \mathrm{ksa}^{2} \mathrm{kspa} a^{5}$ rhoC rhoP jnp_jnksajnp_jnkspa $+2 k s a^{2} k s p a^{4} n^{2}$ rho $C^{2} h n p \_h n k s a$

$-2 k s a^{2} k s p a^{4} n^{2}$ rho $C^{2}$ jnp_jnksa $-4 k s a^{2} k s p a^{4} n^{2}$ rhoC rhoP hnp_hnksa

$+4 \mathrm{ksa}^{2} \mathrm{kspa} \mathrm{a}^{4} n^{2}$ rhoC rhoP jnp_jnksa $-8 \mathrm{ksa}^{2} \mathrm{kspa} \mathrm{a}^{3} \mathrm{n}^{2}$ rhoC rhoP hnp_hnksajnp_jnkspa

$+8 k s a^{2} k s p a^{3} n^{2}$ rhoC rhoP jnp_jnksa jnp_jnkspa $+8 k s a^{2} k s p a^{2} n^{4}$ rho C rhoP hnp_hnksa

$-8 \mathrm{ksa}^{2} \mathrm{kspa}^{2} \mathrm{n}^{4}$ rhoC rhoP jnp_jnksa $+4 \mathrm{kspa} \mathrm{a}^{5} \mathrm{n}^{2} \mathrm{rhoC^{2 }}$ hnp_hnksajnp_jnkspa

$-4 k s p a^{5} n^{2}$ rho $C^{2} j n p \_j n k s a j n p \_j n k s p a-4 k s p a^{4} n^{4}$ rho $C^{2} h n p \_h n k s a$

$+4 k s p a^{4} n^{4} r h o C^{2} j n p \_j n k s a-8 k s a^{4} k s p a n$ rhoP $P^{2}$ hnp_hnksajnp_jnkspa

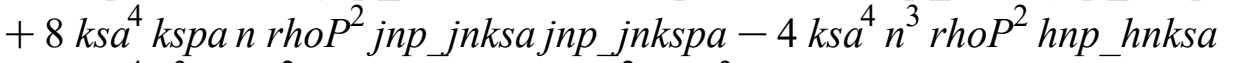

$+4 \mathrm{ksa}^{4} \mathrm{n}^{3} \mathrm{rhoP^{2 }}$ jnp_jnksa $+16 \mathrm{ksa}^{2} \mathrm{kspa}^{3} \mathrm{n}$ rhoC rhoP hnp_hnksajnp_jnkspa

$-16 \mathrm{ksa}^{2} \mathrm{kspa} \mathrm{a}^{3}$ n rhoC rhoP jnp_jnksa jnp_jnkspa $+8 \mathrm{ksa} a^{2} \mathrm{kspa} \mathrm{a}^{2} \mathrm{n}^{3}$ rhoC rhoP hnp_hnksa

$-8 \mathrm{ksa}^{2} \mathrm{kspa}^{2} \mathrm{n}^{3}$ rhoC rhoP jnp_jnksa - $8 \mathrm{kspa} a^{5}$ n rho ${ }^{2}$ hnp_hnksajnp_jnkspa

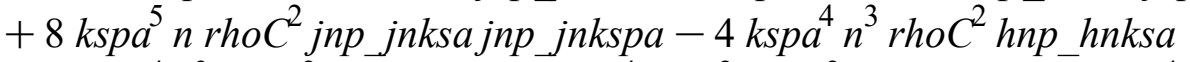

$+4 k s p a^{4} n^{3}$ rho $C^{2} j n p \_j n k s a-2 k s a^{4} k s p a^{2} r h o P^{2} h n p \_h n k s a+2 k s a^{4} k s p a^{2} r h o P^{2} j n p \_j n k s a$

$+8 k s a^{4} n^{2} r h o P^{2} h n p \_h n k s a-8 k s a^{4} n^{2} r h o P^{2} j n p \_j n k s a$

$+2 \mathrm{ksa}^{2} \mathrm{kspa}^{4}$ rhoC rhoP hnp_hnksa $-2 \mathrm{ksa}^{2} \mathrm{kspa}^{4}$ rhoC rhoP jnp_jnksa

$-16 \mathrm{ksa}^{2} \mathrm{kspa}^{2} \mathrm{n}^{2}$ rhoC rhoP hnp_hnksa $+16 \mathrm{ksa}^{2} \mathrm{kspa} \mathrm{a}^{2} \mathrm{n}^{2}$ rhoC rhoP jnp_jnksa

$+8 k s p a^{4} n^{2} r h o C^{2} h n p \_h n k s a-8 k s p a^{4} n^{2}$ rho $\left.C^{2} j n p \_j n k s a\right):$

$>$ denTnSC_: $=-4 \mathrm{ksa}^{5} \mathrm{kspan}^{4} \mathrm{rhoP}^{2}$ hnp_hnksajnp_jnkspa

$+8 \mathrm{ksa}^{3} \mathrm{kspa}^{3} \mathrm{n}^{4}$ rhoC rhoP hnp_hnksajnp_jnkspa

-4 ksakspa $n^{4}{ }^{4}$ rho ${ }^{2}$ hnp_hnksajnp_jnkspa $+k s a^{5} k s p a^{4} n$ rhoC rhoP hnp_hnksa

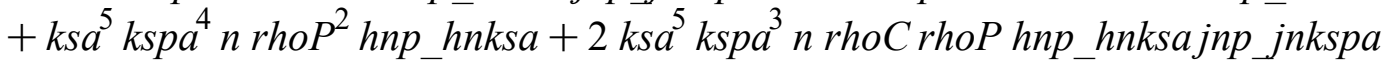

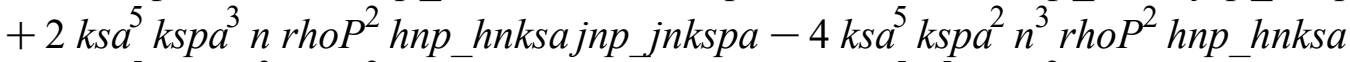

$-8 k s a^{5} k s p a n^{3} r h o P^{2} h n p \_h n k s a j n p \_j n k s p a+4 k s a^{5} n^{5} r h o P^{2} h n p \_h n k s a$

- ksa $a^{4}$ spa $a^{5}$ rho ${ }^{2}$ jnp_jnkspa - ksa $a^{4}$ spa $a^{5}$ rhoC rhoP jnp jnkspa

$-4 k s a^{4} k s p a^{3} n^{3}$ rhoC rhoP jnp_jnkspa $-4 k s a^{4} k s p a n^{5}$ rhoP $P^{2}$ jnp_jnkspa

$-2 \mathrm{ksa}^{3} \mathrm{kspa}^{5}$ n rho $\mathrm{C}^{2}$ hnp_hnksajnp_jnkspa

$-2 \mathrm{ksa}^{3} \mathrm{kspa} a^{5}$ n rhoC rhoP hnp_hnksajnp_jnkspa $+4 k s a^{3} k s p a^{4} n^{3}$ rhoCrhoP hnp_hnksa

$+16 \mathrm{ksa}^{3} \mathrm{kspa}^{3} \mathrm{n}^{3}$ rhoCrhoP hnp_hnksajnp_jnkspa $-8 \mathrm{ksa} a^{3} \mathrm{kspa}^{2} \mathrm{n}^{5}$ rhoC rhoP hnp_hnksa

$+4 k s a^{2} k s p a^{5} n^{3}$ rho $C^{2}$ jnp jnkspa $+8 k s a^{2} k s p a^{3} n^{5}$ rhoC rhoP jnp jnkspa

$-8 k s a k s p a^{5} n^{3}$ rho $C^{2}$ hnp_hnksajnp_jnkspa $+4 k s a k s p a^{4} n^{5}$ rho $C^{2} h n p \_h n k s a$

$-4 k s p a^{5} n^{5} r h o C^{2} j n p \_j n k s p a+k s a^{5} k s p a^{4}$ rhoP $P^{2}$ hnp_hnksa

$+2 k s a^{5} k s p a^{3}$ rho $P^{2}$ hnp_hnksajnp_jnkspa $-2 k s a^{5} k s p a^{2} n^{2}$ rhoC rhoP hnp_hnksa

$-4 k s a^{5} k s p a^{2} n^{2} r h o P^{2} h n p \_h n k s a+4 k s a^{5} k s p a n^{2} r h o P^{2} h n p \_h n k s a j n p \_j n k s p a$

$+8 k s a^{5} n^{4} r h o P^{2} h n p \_h n k s a-k s a^{4} k s p a^{5}$ rhoC rhoP jnp jnkspa $+k s a^{4} k s p a^{4} n^{2}$ rho $C^{2}$

$-2 k s a^{4} k s p a^{4} n^{2}$ rho C rhoP $+k s a^{4} k s p a^{4} n^{2} r h o P^{2}-8 k s a^{4} k s p a^{3} n^{2}$ rhoC rhoP jnp_jnkspa 
$+2 k s a^{4} k s p a^{3} n^{2} r h o P^{2} j n p j n k s p a+4 k s a^{4} k s p a^{2} n^{4} r h o C r h o P-4 k s a^{4} k s p a^{2} n^{4} r h o P^{2}$

$-12 k_{s} a^{4} k s p a n^{4} r h o P^{2}$ jnp jnkspa $+4 k s a^{4} n^{6} r h o P^{2}$

$-2 \mathrm{ksa}^{3} \mathrm{kspa} \mathrm{a}^{5}$ rhoC rhoP hnp_hnksajnp_jnkspa $+2 \mathrm{ksa}^{3} \mathrm{kspa} \mathrm{a}^{4} \mathrm{n}^{2}$ rho $\mathrm{C}^{2}$ hnp_hnksa

$+4 \mathrm{ksa}^{3} \mathrm{kspa} \mathrm{a}^{2} \mathrm{n}^{2}$ rhoC rhoP hnp hnksa $-8 \mathrm{ksa}^{3} \mathrm{kspa}^{3} \mathrm{n}^{2}$ rhoC rhoP hnp_hnksajnp jnkspa

$-16 \mathrm{ksa}^{3} \mathrm{kspa}^{2} n^{4}$ rhoCrhoP hnp_hnksa $+8 \mathrm{ksa}^{2} \mathrm{kspa}^{5} n^{2}$ rho $\mathrm{C}^{2}$ jnp_jnkspa

$-2 k s a^{2} k s p a^{5} n^{2}$ rhoC rhoP jnp jnkspa $-4 k s a^{2} k s p a^{4} n^{4} r h o C^{2}+4 k s a^{2} k s p a^{4} n^{4} r h o C r h o P$

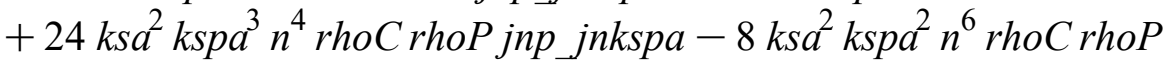

$+4 k s a k s p a^{5} n^{2} r h o C^{2} h n p \_h n k s a j n p \_j n k s p a+8 k s a k s p a^{4} n^{4} r h o C^{2} h n p \_h n k s a$

$-12 k s p a^{5} n^{4} r h o C^{2} j n p \_j n k s p a+4 k s p a^{4} n^{6} r h o C^{2}+2 k s a^{5} k s p a^{2} n$ rho $P^{2} h n p \_h n k s a$

$+8 k s a^{5}$ kspa $n$ rho $P^{2}$ hnp_hnksajnp jnkspa $-4 k s a^{5} n^{3} r h o P^{2} h n p \_h n k s a$

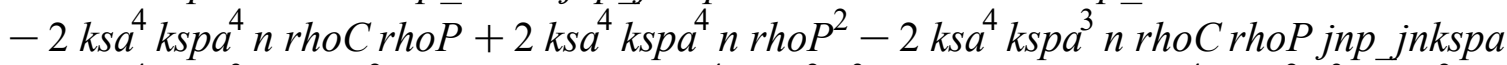

$+4 k s a^{4} k s p a^{3} n r h o P^{2}$ jnp jnkspa $+8 k s a^{4} k s p a^{2} n^{3}$ rhoC rhoP $-8 k s a^{4} k s p a^{2} n^{3} r h o P^{2}$

$-4 k s a^{4} k s p a n^{3} r h o P^{2} j n p \_j n k s p a+12 k s a^{4} n^{5} r h o P^{2}-2 k s a^{3} k s p a^{4} n$ rhoC rhoP hnp_hnksa

$-16 \mathrm{ksa}^{3} \mathrm{kspa}^{3} \mathrm{n}$ rhoCrhoP hnp_hnksajnp_jnkspa $+8 \mathrm{ksa}^{3} \mathrm{kspa}^{2} \mathrm{n}^{3}$ rhoCrhoP hnp_hnksa

$+2 k s a^{2} k s p a^{5} n r h o C^{2} j n p$ jnkspa $-4 k s a^{2} k s p a^{5} n$ rhoC rhoP jnp jnkspa

$-8 k s a^{2} k s p a^{4} n^{3} r h o C^{2}+8 k s a^{2} k s p a^{4} n^{3} r h o C r h o P+8 k s a^{2} k s p a^{3} n^{3}$ rhoC rhoP jnp jnkspa

$-24 k s a^{2} k s p a^{2} n^{5}$ rhoCrhoP $+8 k s a k s p a^{5} n$ rho $C^{2}$ hnp_hnksajnp_jnkspa

$-4 k s a k s p a^{4} n^{3}$ rho $C^{2}$ hnp_hnksa $-4 k s p a^{5} n^{3} r h o C^{2} j n p \_j n k s p a+12 k s p a^{4} n^{5} r h o C^{2}$

$+2 k s a^{5} k s p a^{2} r h o P^{2} h n p \_h n k s a-8 k s s^{5} n^{2} r h o P^{2} h n p \_h n k s a-k s a^{4} k s p a^{4}$ rhoC rhoP

$+k s a^{4} k s p a^{4} r h o P^{2}+2 k s a^{4} k s p a^{3} r h o P^{2} j n p$ jnkspa $+2 k s a^{4} k s p a^{2} n^{2}$ rhoC rhoP

$-2 k s a^{4} k s p a^{2} n^{2} r h o P^{2}+12 k s a^{4} k s p a n^{2} r h o P^{2} j n p j n k s p a+4 k s a^{4} n^{4} r h o P^{2}$

$-2 \mathrm{ksa}^{3} \mathrm{kspa} a^{4}$ rhoC rhoP hnp_hnksa $+16 \mathrm{ksa}^{3} \mathrm{kspa}^{2} \mathrm{n}^{2}$ rhoC rhoP hnp_hnksa

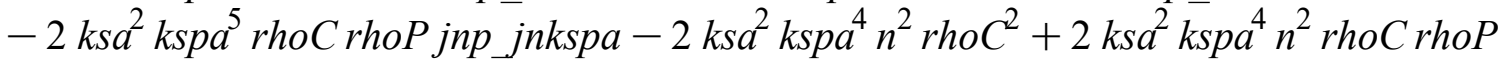

$-24 k s a^{2} k s p a^{3} n^{2}$ rhoCrhoP jnp jnkspa $-8 k s a^{2} k s p a^{2} n^{4}$ rhoC rhoP

$-8 k s a k s p a^{4} n^{2}$ rho $C^{2}$ hnp hnksa $+12 k s p a^{5} n^{2}$ rho $C^{2}$ jnp jnkspa $+4 k s p a^{4} n^{4} r h o C^{2}$

$+4 k s a^{4} k s p a^{2} n r h o P^{2}+8 k s a^{4} k s p a n r h o P^{2} j n p j$ jnkspa $-12 k s a^{4} n^{3} r h o P^{2}$

$-4 k s a^{2} k s p a^{4} n$ rhoC rhoP $-16 k s a^{2} k s p a^{3} n$ rhoC rhoP jnp jnkspa

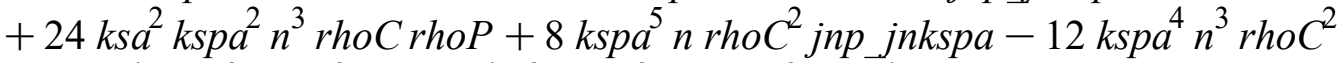

$+2 k s a^{4} k s p a^{2} r h o P^{2}-8 k s a^{4} n^{2} r h o P^{2}-2 k s a^{2} k s p a^{4}$ rhoC rhoP

$+16 \mathrm{ksa}^{2} \mathrm{kspa}^{2} \mathrm{n}^{2}$ rhoCrhoP $-8 \mathrm{kspa} \mathrm{a}^{4} \mathrm{n}^{2} \mathrm{rhoC^{2 }}$ :

$>$ denTnSC_ - denTnCC_\# confirm the denominator is identical to that of TnCC

TnSSLeadkca_: $($ series('leadterm' $(($ TnSS_ $)), k c a, 1))$

\#initially show output, then copy numerator and denominator and then hide output

TnSSLeadkca_: $=-\left(-4 \mathrm{ksa}^{5} \mathrm{kspa}^{4}{ }^{4} \mathrm{rhoP}^{2}\right.$ jnp_jnksa jnp_jnkspa

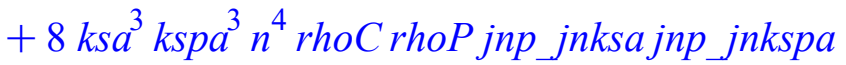

$-4 k s a k s p a^{5} n^{4} r h o C^{2} j n p \_j n k s a j n p \_j n k s p a+k s a^{5} k s p a^{4} n$ rhoC rhoP jnp jnksa

$+k s a^{5} k s p a^{4} n r h o P^{2} j n p$ jnksa $+2 k s a^{5} k s p a^{3} n$ rhoC rhoP jnp_jnksa jnp jnkspa

$+2 k s a^{5} k s p a^{3} n r h o P^{2}$ jnp jnksa jnp jnkspa $-4 k s a^{5} k s p a^{2} n^{3} r h o P^{2}$ jnp jnksa

$-8 k s a^{5} k s p a n^{3} r h o P^{2} j n p \_j n k s a j n p \_j n k s p a+4 k s a^{5} n^{5} r h o P^{2} j n p$ jnksa

$-k s a^{4} k s p a^{5} n r h o C^{2} j n p$ jnkspa $-k s a^{4} k s p a^{5} n$ rhoC rhoP jnp jnkspa 
$-4 k s a^{4} k s p a^{3} n^{3}$ rhoC rhoP jnp_jnkspa $-4 k s a^{4} k s p a n^{5}$ rhoP ${ }^{2}$ jnp_jnkspa

$-2 k s a^{3} k s p a^{5} n$ rho $C^{2}$ jnp_jnksa jnp_jnkspa

$-2 \mathrm{ksa}^{3} \mathrm{kspa}^{5}$ n rhoC rhoP jnp_jnksa jnp_jnkspa $+4 k s a^{3} k s p a^{4} n^{3}$ rhoC rhoP jnp_jnksa

$+16 \mathrm{ksa}^{3} \mathrm{kspa}^{3} \mathrm{n}^{3}$ rhoC rhoP jnp_jnksa jnp_jnkspa $-8 \mathrm{ksa}^{3} \mathrm{kspa}^{2} \mathrm{n}^{5}$ rhoC rhoP jnp_jnksa

$+4 k s a^{2} k s p a^{5} n^{3} r h o C^{2} j n p \_j n k s p a+8 k s a^{2} k s p a^{3} n^{5}$ rhoC rhoP jnp_jnkspa

$-8 k s a k s p a^{5} n^{3} r h o C^{2} j n p \_j n k s a j n p \_j n k s p a+4 k s a k s p a^{4} n^{5} r h o C^{2} j n p \_j n k s a$

$-4 k s p a^{5} n^{5} r h o C^{2} j n p \_j n k s p a+k s a^{5} k s p a^{4} r h o P^{2} j n p \_j n k s a$

$+2 k s a^{5} k s p a^{3} r h o P^{2}$ jnp_jnksa jnp_jnkspa $-2 k s a^{5} k s p a^{2} n^{2}$ rhoC rhoP jnp_jnksa

$-4 k s a^{5} k s p a^{2} n^{2} r h o P^{2} j n p \_j n k s a+4 k s a^{5} k s p a n^{2} r h o P^{2} j n p \_j n k s a j n p \_j n k s p a$

$+8 k s a^{5} n^{4} r h o P^{2} j n p \_j n k s a-k s a^{4} k s p a^{5}$ rhoC rhoP jnp jnkspa $+k s a^{4} k s p a^{4} n^{2} r h o C^{2}$

$-2 k s a^{4} k s p a^{4} n^{2}$ rhoC rhoP $+k s a^{4} k s p a^{4} n^{2} r h o P^{2}-8 k s a^{4} k s p a^{3} n^{2}$ rhoC rhoP jnp jnkspa

$+2 k s a^{4} k s p a^{3} n^{2} r h o P^{2} j n p \_j n k s p a+4 k s a^{4} k s p a^{2} n^{4}$ rhoC rhoP $-4 k s a^{4} k s p a^{2} n^{4} r h o P^{2}$

$-12 k s a^{4} k s p a n^{4} r h o P^{2} j n p \_j n k s p a+4 k s a^{4} n^{6} r h o P^{2}$

$-2 \mathrm{ksa}^{3} \mathrm{kspa} \mathrm{a}^{5}$ rhoC rhoP jnp_jnksa jnp_jnkspa $+2 k s a^{3} k s p a^{4} n^{2}$ rho $C^{2}$ jnp_jnksa

$+4 k s a^{3} k s p a^{4} n^{2}$ rhoC rhoP jnp_jnksa $-8 k s a^{3} k s p a^{3} n^{2}$ rhoC rhoP jnp_jnksa jnp_jnkspa

$-16 \mathrm{ksa}^{3} \mathrm{kspa} \mathrm{a}^{2} n^{4}$ rhoC rhoP jnp_jnksa $+8 k s a^{2} k s p a^{5} n^{2}$ rho $C^{2} j n p \_j n k s p a$

$-2 k s a^{2} k s p a^{5} n^{2}$ rhoC rhoP jnp jnkspa $-4 k s a^{2} k s p a^{4} n^{4} r h o C^{2}+4 k s a^{2} k s p a^{4} n^{4}$ rhoC rhoP

$+24 k s a^{2} k s p a^{3} n^{4}$ rhoC rhoP jnp_jnkspa $-8 k s a^{2} k s p a^{2} n^{6}$ rhoC rhoP

$+4 k s a k s p a^{5} n^{2} r h o C^{2} j n p \_j n k s a j n p \_j n k s p a+8 k s a k s p a^{4} n^{4} r h o C^{2} j n p \_j n k s a$

$-12 k s p a^{5} n^{4} r h o C^{2} j n p \_j n k s p a+4 k s p a^{4} n^{6} r h o C^{2}+2 k s a^{5} k s p a^{2} n r h o P^{2} j n p$ jnksa

$+8 k s a^{5} k s p a n$ rhoP $P^{2}$ jnp_jnksa jnp_jnkspa $-4 k s a^{5} n^{3}$ rhoP $P^{2}$ jnp_jnksa

$-2 \mathrm{ksa}^{4} \mathrm{kspa}^{4}$ n rhoC rhoP $+2 \mathrm{ksa}^{4} \mathrm{kspa}^{4} \mathrm{n}$ rhoP $\mathrm{P}^{2}-2 \mathrm{ksa} \mathrm{kspa}^{3}$ n rhoC rhoP jnp_jnkspa

$+4 k s a^{4} k s p a^{3} n r h o P^{2} j n p \_j n k s p a+8 k s a^{4} k s p a^{2} n^{3}$ rhoCrhoP $-8 k s a^{4} k s p a^{2} n^{3}$ rho $P^{2}$

$-4 k s a^{4} k s p a n^{3} r_{h o P^{2}} j n p \_j n k s p a+12 k s a^{4} n^{5} r h o P^{2}-2 k s a^{3} k s p a^{4} n$ rhoC rhoP jnp_jnksa

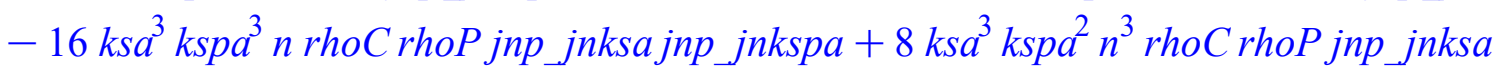

$+2 \mathrm{ksa}^{2} \mathrm{kspa}^{5} \mathrm{n}$ rho ${ }^{2}$ jnp_jnkspa $-4 \mathrm{ksa}^{2} \mathrm{kspa}^{5}$ n rhoC rhoP jnp_jnkspa

$-8 k s a^{2} k s p a^{4} n^{3} r h o C^{2}+8 k s a^{2} k s p a^{4} n^{3}$ rhoC rhoP $+8 k s a^{2} k s p a^{3} n^{3}$ rhoC rhoP jnp_jnkspa

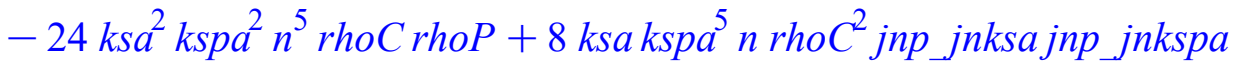

$-4 k s a k s p a^{4} n^{3} r h o C^{2} j n p \_j n k s a-4 k s p a^{5} n^{3} r h o C^{2} j n p \_j n k s p a+12 k s p a^{4} n^{5} r h o C^{2}$

$+2 k s a^{5} k s p a^{2} r h o P^{2} j n p \_j n k s a-8 k s a^{5} n^{2} r h o P^{2} j n p \_j n k s a-k s a^{4} k s p a^{4}$ rhoC rhoP

$+k s a^{4} k s p a^{4} r h o P^{2}+2 k s a^{4} k s p a^{3} r h o P^{2} j n p \_j n k s p a+2 k s a^{4} k s p a^{2} n^{2}$ rhoC rhoP

$-2 k s a^{4} k s p a^{2} n^{2} r h o P^{2}+12 k s a^{4} k s p a n^{2} r h o P^{2} j n p \_j n k s p a+4 k s a^{4} n^{4} r h o P^{2}$

$-2 \mathrm{ksa}^{3} \mathrm{kspa} \mathrm{a}^{4}$ rhoC rhoP jnp_jnksa $+16 \mathrm{ksa}^{3} \mathrm{kspa}^{2} \mathrm{n}^{2}$ rhoC rhoP jnp_jnksa

$-2 k s a^{2} k s p a^{5}$ rhoC rhoP jnp jnkspa $-2 k s a^{2} k s p a^{4} n^{2}$ rho $C^{2}+2 k s a^{2} k s p a^{4} n^{2}$ rhoC rhoP

$-24 k s a^{2} k s p a^{3} n^{2}$ rhoC rhoP jnp_jnkspa $-8 \mathrm{ksa}^{2} \mathrm{kspa}^{2} n^{4}$ rhoC rhoP

$-8 k s a k s p a^{4} n^{2} r h o C^{2} j n p \_j n k s a+12 k s p a^{5} n^{2} r h o C^{2} j n p \_j n k s p a+4 k s p a^{4} n^{4} r h o C^{2}$ 
$+4 k s a^{4} k s p a^{2} n r h o P^{2}+8 k s a^{4} k s p a n r h o P^{2} j n p \_j n k s p a-12 k s a^{4} n^{3} r h o P^{2}$

$-4 \mathrm{ksa}^{2} \mathrm{kspa} \mathrm{a}^{4} \mathrm{n}$ rhoC rhoP - $16 \mathrm{ksa}^{2} \mathrm{kspa}^{3} \mathrm{n}$ rhoC rhoP jnp jnkspa

$+24 k s a^{2} k s p a^{2} n^{3}$ rhoC rhoP $+8 k s p a^{5} n$ rho $C^{2} j n p \_j n k s p a-12 k s p a^{4} n^{3} r h o C^{2}$

$+2 k s a^{4} k s p a^{2} r h o P^{2}-8 k s a^{4} n^{2} r h o P^{2}-2 k s a^{2} k s p a^{4}$ rhoC rhoP

$+16 k s a^{2} k s p a^{2} n^{2}$ rhoC rhoP $\left.-8 k s p a^{4} n^{2} r h o C^{2}\right) /($

-4 ksa $a^{5}$ spa $n^{4}$ rhoP $P^{2}$ hnp_hnksajnp_jnkspa

$+8 \mathrm{ksa}^{3} \mathrm{kspa}^{3} \mathrm{n}^{4}$ rhoC rhoP hnp_hnksajnp_jnkspa

$-4 k s a k s p a^{5} n^{4}$ rho ${ }^{2}$ hnp_hnksajnp_jnkspa $+k s a^{5} k s p a^{4} n$ rhoC rhoP hnp_hnksa

$+k s a^{5} k s p a^{4} n$ rhoP $P^{2}$ hnp_hnksa $+2 k_{s a} a^{5} k s p a^{3} n$ rhoC rhoP hnp_hnksajnp_jnkspa

$+2 k s a^{5} k s p a^{3} n r h o P^{2} h n p \_h n k s a j n p \_j n k s p a-4 k s a^{5} k s p a^{2} n^{3} r h o P^{2} h n p \_h n k s a$

$-8 k s a^{5} k s p a n^{3} r h o P^{2} h n p \_h n k s a j n p \_j n k s p a+4 k s a^{5} n^{5} r h o P^{2}$ hnp_hnksa

$-k s a^{4} k s p a^{5} n$ rho $C^{2} j n p \_j n k s p a-k s a^{4} k s p a^{5} n$ rhoC rhoP jnp_jnkspa

$-4 k s a^{4} k s p a^{3} n^{3}$ rhoC rhoP jnp_jnkspa $-4 k s a^{4} k s p a n^{5}$ rhoP ${ }^{2}{ }_{j n p} j n k s p a$

$-2 k s a^{3} k s p a^{5} n$ rho $C^{2} h n p \_h n k s a j n p \_j n k s p a$

$-2 k s a^{3} k s p a^{5} n$ rhoC rhoP hnp_hnksajnp_jnkspa $+4 k s a^{3} k s p a^{4} n^{3}$ rhoC rhoP hnp_hnksa

$+16 \mathrm{ksa}^{3} \mathrm{kspa}^{3} \mathrm{n}^{3}$ rhoC rhoP hnp_hnksajnp_jnkspa $-8 \mathrm{ksa} a^{3} \mathrm{kspa} a^{2} n^{5}$ rhoC rhoP hnp_hnksa

$+4 k s a^{2} k s p a^{5} n^{3} r h o C^{2} j n p \_j n k s p a+8 k s a^{2} k s p a^{3} n^{5}$ rhoC rhoP jnp_jnkspa

$-8 k s a k s p a^{5} n^{3}$ rho $C^{2} h n p \_h n k s a j n p \_j n k s p a+4 k s a k s p a^{4} n^{5}$ rho $C^{2} h n p \_h n k s a$

$-4 k s p a^{5} n^{5} r h o C^{2} j n p \_j n k s p a+k s a^{5} k s p a^{4} r h o P^{2} h n p \_h n k s a$

$+2 k s a^{5} k s p a^{3}$ rhoP $P^{2} h n p \_h n k s a j n p \_j n k s p a-2 k s a^{5} k s p a^{2} n^{2}$ rhoC rhoP hnp_hnksa

$-4 k s a^{5} k s p a^{2} n^{2} r h o P^{2} h n p \_h n k s a+4 k s a^{5} k s p a n^{2} r h o P^{2} h n p \_h n k s a j n p \_j n k s p a$

$+8 k s a^{5} n^{4}$ rhoP ${ }^{2} h n p \_h n k s a-k s a^{4} k s p a^{5}$ rhoC rhoP jnp_jnkspa $+k s a^{4} k s p a^{4} n^{2} r h o C^{2}$

$-2 k s a^{4} k s p a^{4} n^{2}$ rhoC rhoP $+k s a^{4} k s p a^{4} n^{2} r h o P^{2}-8 k s a^{4} k s p a^{3} n^{2}$ rhoC rhoP jnp jnkspa

$+2 k s a^{4} k s p a^{3} n^{2} r h o P^{2} j n p \_j n k s p a+4 k s a^{4} k s p a^{2} n^{4}$ rhoC rhoP $-4 k s a^{4} k s p a^{2} n^{4} r h o P^{2}$

$-12 \mathrm{ksa}^{4} \mathrm{kspan}{ }^{4} \mathrm{rhoP}^{2}$ jnp_jnkspa $+4 k s a^{4} n^{6} r h o P^{2}$

$-2 \mathrm{ksa}^{3} \mathrm{kspa}^{5}$ rhoCrhoP hnp_hnksajnp_jnkspa $+2 \mathrm{ksa} a^{3} k s p a^{4} n^{2}$ rho $C^{2} h n p \_h n k s a$

$+4 \mathrm{ksa}^{3} \mathrm{kspa}^{4} \mathrm{n}^{2}$ rhoC rhoP hnp_hnksa $-8 \mathrm{ksa}^{3} \mathrm{kspa}^{3} \mathrm{n}^{2}$ rhoC rhoP hnp_hnksajnp_jnkspa

$-16 \mathrm{ksa}^{3} \mathrm{kspa} \mathrm{a}^{2} \mathrm{n}^{4}$ rhoC rhoP hnp_hnksa $+8 \mathrm{ksa} a^{2} \mathrm{kspa} \mathrm{n}^{2} \mathrm{rhoC^{2 }}$ jnp_jnkspa

$-2 k s a^{2} k s p a^{5} n^{2}$ rhoC rhoP jnp_jnkspa $-4 k s a^{2} k s p a^{4} n^{4} r h o C^{2}+4 k s a^{2} k s p a^{4} n^{4}$ rhoC rhoP

$+24 \mathrm{ksa}^{2} \mathrm{kspa} \mathrm{a}^{3} \mathrm{n}^{4}$ rhoC rhoP jnp_jnkspa $-8 \mathrm{ksa}^{2} \mathrm{kspa} \mathrm{a}^{6} \mathrm{rhoC}_{\mathrm{ChoP}}$

$+4 k s a k s p a^{5} n^{2} r h o C^{2} h n p \_h n k s a j n p \_j n k s p a+8 k s a k s p a^{4} n^{4} r h o C^{2} h n p \_h n k s a$

$-12 k s p a^{5} n^{4} r h o C^{2} j n p \_j n k s p a+4 k s p a^{4} n^{6} r h o C^{2}+2 k s a^{5} k s p a^{2} n r h o P^{2} h n p \_h n k s a$

$+8 k s a^{5}$ kspa $n$ rhoP $P^{2}$ hnp_hnksajnp_jnkspa $-4 k s a^{5} n^{3} r h o P^{2}$ hnp_hnksa

$-2 \mathrm{ksa}^{4} \mathrm{kspa}^{4} \mathrm{n}$ rhoC rhoP $+2 \mathrm{ksa}^{4} \mathrm{kspa}^{4} \mathrm{n}$ rhoP $\mathrm{P}^{2}-2 \mathrm{ksa} \mathrm{kspa}^{3}$ n rhoC rhoP jnp jnkspa

$+4 k s a^{4} k s p a^{3} n r h o P^{2} j n p \_j n k s p a+8 k s a^{4} k s p a^{2} n^{3}$ rhoC rhoP $-8 k s a^{4} k s p a^{2} n^{3} r h o P^{2}$

$-4 k s a^{4} k s p a n^{3} r h o P^{2} j n p \_j n k s p a+12 k s a^{4} n^{5} r h o P^{2}$ 
$-2 \mathrm{ksa}^{3} \mathrm{kspa} \mathrm{a}^{4} \mathrm{n}$ rhoC rhoP hnp_hnksa $-16 \mathrm{ksa}^{3} \mathrm{kspa}^{3} \mathrm{n}$ rhoC rhoP hnp_hnksajnp_jnkspa

$+8 \mathrm{ksa}^{3} \mathrm{kspa}^{2} \mathrm{n}^{3}$ rhoC rhoP hnp_hnksa $+2 \mathrm{ksa}^{2} \mathrm{kspa}^{5} n$ rho $\mathrm{C}^{2}$ jnp_jnkspa

$-4 k s a^{2} k s p a^{5} n$ rhoC rhoP jnp jnkspa $-8 k s a^{2} k s p a^{4} n^{3} r h o C^{2}+8 k s a^{2} k s p a^{4} n^{3}$ rhoC rhoP

$+8 k s a^{2} k s p a^{3} n^{3}$ rhoC rhoP jnp jnkspa $-24 k s a^{2} k s p a^{2} n^{5}$ rhoC rhoP

+8 ksakspa $a^{5}$ rho $C^{2}$ hnp_hnksajnp_jnkspa -4 ksa kspa $a^{4} n^{3}$ rhoC ${ }^{2}$ hnp_hnksa

$-4 k s p a^{5} n^{3} r h o C^{2} j n p \_j n k s p a+12 k s p a^{4} n^{5} r h o C^{2}+2 k s a^{5} k s p a^{2} r h o P^{2} h n p \_h n k s a$

$-8 k s a^{5} n^{2} r h o P^{2} h n p \_h n k s a-k s a^{4} k s p a^{4} r h o C r h o P+k s a^{4} k s p a^{4} r h o P^{2}$

$+2 k s a^{4} k s p a^{3} r h o P^{2} j n p j n k s p a+2 k s a^{4} k s p a^{2} n^{2} r h o C r h o P-2 k s a^{4} k s p a^{2} n^{2} r h o P^{2}$

$+12 \mathrm{ksa}^{4} \mathrm{kspan}^{2} \mathrm{rhoP}^{2}$ jnp jnkspa $+4 \mathrm{ksa}^{4} \mathrm{n}^{4} \mathrm{rhoP}^{2}-2 \mathrm{ksa}^{3} \mathrm{kspa} \mathrm{a}^{4}$ rhoC rhoP hnp_hnksa

$+16 \mathrm{ksa}^{3} \mathrm{kspa}^{2} \mathrm{n}^{2}$ rhoC rhoP hnp_hnksa $-2 \mathrm{ksa}^{2} \mathrm{kspa} \mathrm{a}^{5}$ rhoC rhoP jnp_jnkspa

$-2 k s a^{2} k s p a^{4} n^{2} r h o C^{2}+2 k s a^{2} k s p a^{4} n^{2}$ rhoC rhoP

$-24 k s a^{2} k s p a^{3} n^{2}$ rhoC rhoP jnp jnkspa $-8 k s a^{2} k s p a^{2} n^{4}$ rhoC rhoP

$-8 k s a k s p a^{4} n^{2} r h o C^{2} h n p \_h n k s a+12 k s p a^{5} n^{2} r h o C^{2} j n p j$ jnkspa $+4 k s p a^{4} n^{4} r h o C^{2}$

$+4 k s a^{4} k s p a^{2} n r h o P^{2}+8 k s a^{4} k s p a n r h o P^{2} j n p$ jnkspa $-12 k s a^{4} n^{3} r h o P^{2}$

$-4 \mathrm{ksa}^{2} \mathrm{kspa}^{4} \mathrm{n}$ rhoC rhoP $-16 \mathrm{ksa}^{2} \mathrm{kspa}^{3} \mathrm{n}$ rhoC rhoP jnp jnkspa

$+24 k s a^{2} k s p a^{2} n^{3} r h o C r h o P+8 k s p a^{5} n r h o C^{2} j n p j n k s p a-12 k s p a^{4} n^{3} r h o C^{2}$

$+2 k s a^{4} k s p a^{2} r h o P^{2}-8 k s a^{4} n^{2} r h o P^{2}-2 k s a^{2} k s p a^{4} r h o C r h o P$

$\left.+16 \mathrm{ksa}^{2} k s p a^{2} n^{2} r h o C r h o P-8 k s p a^{4} n^{2} r h o C^{2}\right)$

$>$ numTnSS_ $:=-\left(-4 k s a^{5} k s p a n^{4}{ }^{2} h o P^{2}\right.$ jnp jnksa jnp_jnkspa

$+8 \mathrm{ksa}^{3} \mathrm{kspa} \mathrm{n}^{3} \mathrm{n}^{\mathrm{r}} \mathrm{rhoC}$ rhoP jnp jnksa jnp jnkspa

-4 ksa kspa ${ }^{5} n^{4}$ rho $C^{2}$ jnp jnksa jnp jnkspa $+k s a^{5} k s p a^{4} n$ rhoC rhoP jnp jnksa

$+k s a^{5} k s p a^{4} n r h o P^{2} j n p$ jnks $n a+2 k s a^{5} k s p a^{3} n$ rhoC rhoP jnp jnksa jnp jnkspa

$+2 k_{s a}^{5} k s p a^{3} n$ rho $P^{2}$ jnp jnksa jnp jnkspa $-4 k s a^{5} k s p a^{2} n^{3} r h o P^{2}$ jnp jnkssa

$-8 k s a^{5} k s p a n^{3} r h o P^{2}$ jnp_jnksa jnp_jnkspa $+4 k s a^{5} n^{5} r h o P^{2}$ jnp jnks

$-k s a^{4} k s p a^{5} n$ rho ${ }^{2}$ jnp jnkspa - ksa $k s p a^{5} n$ rhoC rhoP jnp jnkspa

$-4 k s a^{4} k s p a^{3} n^{3}$ rhoC rhoP jnp jnkspa $-4 k s a^{4} k s p a n^{5}$ rhoP $P^{2}$ jnp jnkspa

$-2 k_{s a^{3}} k s p a^{5} n$ rho $C^{2}$ jnp jnksa jnp jnkspa

$-2 \mathrm{ksa}^{3} \mathrm{kspa}^{5}$ n rhoC rhoP jnp jnksa jnp jnkspa $+4 \mathrm{ksa}^{3} \mathrm{kspa}^{4} n^{3}$ rhoC rhoP jnp jnksa

$+16 \mathrm{ksa}^{3} \mathrm{kspa}^{3} \mathrm{n}^{3}$ rhoCrhoP jnp jnksa jnp jnkspa $-8 \mathrm{ksa}^{3} \mathrm{kspa}^{2} n^{5}$ rhoC rhoP jnp jnksa

$+4 k s a^{2} k s p a^{5} n^{3} r h o C^{2} j n p$ jnkspa $+8 k_{s a^{2}} k_{s p a}{ }^{3} n^{5}$ rhoC rhoP jnp jnkspa

$-8 k s a k s p a^{5} n^{3} r h o C^{2}$ jnp jonksa jnp jnkspa $+4 k s a k s p a^{4} n^{5} r h o C^{2} j n p$ jnksa

$-4 k s p a^{5} n^{5} r h o C^{2}$ jnp jnkspa $+k s a^{5} k s p a^{4} r h o P^{2}$ jnp jnksa

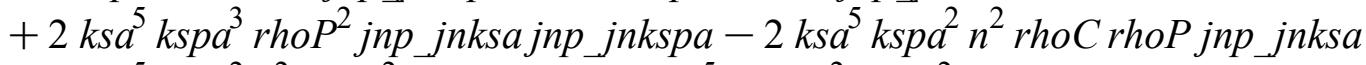

$-4 k s a^{5} k s p a^{2} n^{2} r h o P^{2} j n p \_j n k s a+4 k s a^{5} k s p a n^{2} r h o P^{2}$ jnp_jnksa jnp_jnkspa

$+8 k s a^{5} n^{4} r h o P^{2} j n p$ jnksa $-k s a^{4} k s p a^{5}$ rhoC rhoP jnp jnkspa $+k s a^{4} k s p a^{4} n^{2} r h o C^{2}$

$-2 k s a^{4} k s p a^{4} n^{2}$ rhoC rhoP $+k s a^{4} k s p a^{4} n^{2} r h o P^{2}-8 k s a^{4} k s p a^{3} n^{2}$ rhoC rhoP jnp jnkspa

$+2 k s a^{4} k s p a^{3} n^{2} r h o P^{2} j n p \_j n k s p a+4 k s a^{4} k s p a^{2} n^{4} r h o C r h o P-4 k s a^{4} k s p a^{2} n^{4} r h o P^{2}$

$-12 k s a^{4} k s p a n^{4} r h o P^{2} j n p$ jnkspa $+4 k s a^{4} n^{6} r h o P^{2}$

$-2 k s a^{3} k s p a^{5}$ rhoC rhoP jnp_jnksa jnp jnkspa $+2 k s a^{3} k s p a^{4} n^{2} r h o C^{2} j n p \_j n k s a$ 
$+4 k s a^{3} k s p a^{4} n^{2}$ rhoC rhoP jnp jnksa $-8 k s a^{3} k s p a^{3} n^{2}$ rhoC rhoP jnp_jnksa jnp_jnkspa $-16 \mathrm{ksa}^{3} \mathrm{kspa}^{2} n^{4}$ rhoCrhoP jnp jnksa $+8 k s a^{2} k s p a^{5} n^{2}$ rho $C^{2}$ jnp jnkspa

$-2 k s a^{2} k s p a^{5} n^{2}$ rhoC rhoP jnp jnkspa $-4 k s a^{2} k s p a^{4} n^{4} r h o C^{2}+4 k s a^{2} k s p a^{4} n^{4}$ rhoC rhoP $+24 \mathrm{ksa}^{2} \mathrm{kspa}^{3} n^{4}$ rhoCrhoP jnp jnkspa $-8 \mathrm{ksa}^{2} \mathrm{kspa}^{2} n^{6}$ rhoC rhoP $+4 k s a k s p a^{5} n^{2} r h o C^{2}$ jnp _jnksa jnp_jnkspa $+8 k s a k s p a^{4} n^{4} r h o C^{2} j n p \_j n k s a$ $-12 k s p a^{5} n^{4} r h o C^{2}$ jnp jnkspa $+4 k s p a^{4} n^{6} r h o C^{2}+2 k s a^{5} k s p a^{2} n r h o P^{2} j n p$ jnksa $+8 k s a^{5} k s p a n$ rhoP $P^{2}$ jnp jnksa jnp jnkspa $-4 k s a^{5} n^{3} r h o P^{2}$ jnp jnksa $-2 \mathrm{ksa}^{4} \mathrm{kspa}^{4} \mathrm{n}$ rhoC rhoP $+2 \mathrm{ksa}^{4} \mathrm{kspa}^{4} \mathrm{n}$ rho $\mathrm{P}^{2}-2 \mathrm{ksa}^{4} \mathrm{kspa}^{3} \mathrm{n}$ rhoC rhoP jnp jnkspa $+4 k s a^{4} k s p a^{3} n r h o P^{2} j n p$ jnkspa $+8 k s a^{4} k s p a^{2} n^{3}$ rhoC rhoP $-8 k s a^{4} k s p a^{2} n^{3} r h o P^{2}$

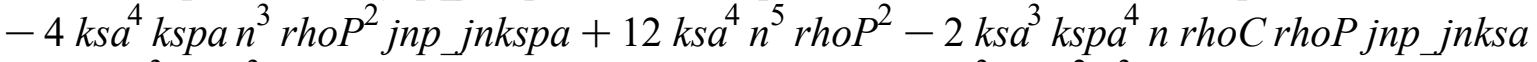
$-16 \mathrm{ksa}^{3} \mathrm{kspa} a^{3}$ n rhoC rhoP jnp jnksa jnp_jnkspa $+8 \mathrm{ksa}^{3} \mathrm{kspa}^{2} \mathrm{n}^{3}$ rhoC rhoP jnp jnksa $+2 \mathrm{ksa}^{2} k s p a^{5} n$ rho $C^{2}$ jnp jnkspa $-4 \mathrm{ksa}^{2} \mathrm{kspa}^{5}$ n rhoC rhoP jnp jnkspa $-8 k s a^{2} k s p a^{4} n^{3} r h o C^{2}+8 k s a^{2} k s p a^{4} n^{3} r h o C r h o P+8 k s a^{2} k s p a^{3} n^{3}$ rhoC rhoP jnp jnkspa

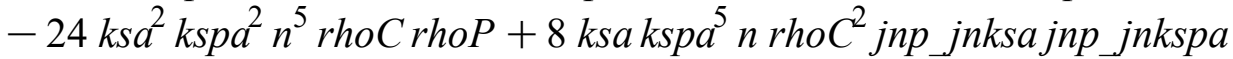
$-4 k s a k s p a^{4} n^{3} r h o C^{2} j n p$ jnksa $-4 k s p a^{5} n^{3} r h o C^{2} j n p j n k s p a+12 k s p a^{4} n^{5} r h o C^{2}$ $+2 k s a^{5} k s p a^{2} r h o P^{2} j n p \_j n k s a-8 k s a^{5} n^{2} r h o P^{2} j n p \_j n k s a-k s a^{4} k s p a^{4}$ rhoC rhoP $+k s a^{4} k s p a^{4} r h o P^{2}+2 k s a^{4} k s p a^{3} r h o P^{2} j n p j n k s p a+2 k s a^{4} k s p a^{2} n^{2} r h o C r h o P$ $-2 k s a^{4} k s p a^{2} n^{2} r h o P^{2}+12 k s a^{4} k s p a n^{2} r h o P^{2} j n p$ jnkspa $+4 k s a^{4} n^{4} r h o P^{2}$

$-2 \mathrm{ksa}^{3} \mathrm{kspa} \mathrm{a}^{4}$ rhoC rhoP jnp jnksa $+16 \mathrm{ksa}^{3} \mathrm{kspa}^{2} \mathrm{n}^{2}$ rhoC rhoP jnp jnksa

$-2 k s a^{2} k s p a^{5}$ rhoC rhoP jnp jnkspa $-2 k s a^{2} k s p a^{4} n^{2} r h o C^{2}+2 k s a^{2} k s p a^{4} n^{2}$ rhoC rhoP

$-24 k s a^{2} k s p a^{3} n^{2}$ rhoCrhoP jnp jnkspa $-8 k^{2} a^{2} k s p a^{2} n^{4}$ rhoC rhoP

$-8 k s a k s p a^{4} n^{2} r h o C^{2} j n p$ jnksa $+12 k s p a^{5} n^{2} r h o C^{2} j n p$ jnkspa $+4 k s p a^{4} n^{4} r h o C^{2}$

$+4 k s a^{4} k s p a^{2} n r h o P^{2}+8 k s a^{4} k s p a n r h o P^{2} j n p j n k s p a-12 k s a^{4} n^{3} r h o P^{2}$

$-4 \mathrm{ksa}^{2} \mathrm{kspa}^{4}$ n rhoC rhoP $-16 \mathrm{ksa}^{2} \mathrm{kspa}^{3}$ n rhoC rhoP jnp_jnkspa

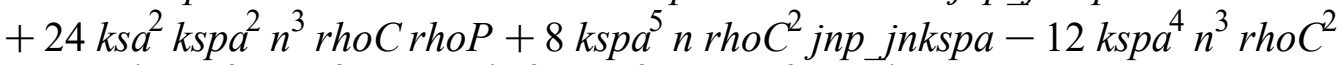

$+2 k s a^{4} k s p a^{2} r h o P^{2}-8 k s a^{4} n^{2} r h o P^{2}-2 k s a^{2} k s p a^{4}$ rhoC rhoP

$+16 k s a^{2} k s p a^{2} n^{2}$ rhoCrhoP $\left.-8 k s p a^{4} n^{2} r h o C^{2}\right)$ :

$>$ denTnSS_ $:=\left(-4 \mathrm{ksa}^{5} \mathrm{kspan}^{4} \mathrm{rhoP}^{2}\right.$ hnp_hnksajnp_jnkspa

$+8 \mathrm{ksa}^{3} \mathrm{kspa}^{3} \mathrm{n}^{4}$ rhoCrhoP hnp_hnksajnp_jnkspa

$-4 k s a k s p a^{5} n^{4}$ rho $C^{2}$ hnp_hnksa jnp_jnkspa + ksa $a^{5} k s p a^{4} n$ rhoC rhoP hnp_hnksa

$+k s a^{5} k s p a^{4} n$ rhoP $P^{2} h n p \_h n k s a+2 k s a^{5} k s p a^{3} n$ rhoC rhoP hnp_hnksajnp_jnkspa

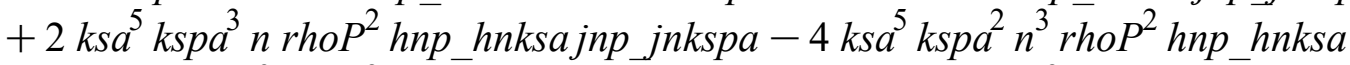

$-8 k s a^{5} k s p a n^{3}$ rhoP $P^{2}$ hnp_hnksajnp_jnkspa $+4 k s a^{5} n^{5} r h o P^{2}$ hnp_hnksa

$-k s a^{4} k s p a^{5} n$ rho $C^{2}$ jnp jnkspa - ksa kspa $n$ rhoC rhoP jnp jnkspa

$-4 k s a^{4} k s p a^{3} n^{3}$ rhoC rhoP jnp jnkspa $-4 k s a^{4} k s p a n^{5}$ rhoP $P^{2}$ jnp jnkspa

$-2 \mathrm{ksa}^{3} \mathrm{kspa}^{5}$ n rho ${ }^{2}$ hnp_hnksajnp_jnkspa

$-2 k_{s a^{3}} k_{s p a} a^{5} n$ rhoC rhoP hnp_hnksajnp_jnkspa $+4 k_{s} a^{3} k s p a^{4} n^{3}$ rhoC rhoP hnp_hnksa

$+16 \mathrm{ksa}^{3} \mathrm{kspa}^{3} \mathrm{n}^{3}$ rhoCrhoP hnp_hnksajnp jnkspa $-8 \mathrm{ksa}^{3} \mathrm{kspa}^{2} \mathrm{n}^{5}$ rhoC rhoP hnp_hnksa $+4 \mathrm{ksa}^{2} k s p a^{5} n^{3} r h o C^{2} j n p$ jnkspa $+8 \mathrm{ksa}^{2} \mathrm{kspa}^{3} n^{5}$ rhoC rhoP jnp jnkspa

$-8 k s a k s p a^{5} n^{3} r h o C^{2} h n p$ hnksajnp_jnkspa +4 ksakspa $n^{5}{ }^{5} h o C^{2} h n p \_h n k s a$

$-4 k s p a^{5} n^{5} r h o C^{2}$ jnp jnkspa $+k s a^{5} k s p a^{4} r h o P^{2} h n p \_h n k s a$

$+2 k s a^{5} k s p a^{3} r h o P^{2} h n p \_h n k s a j n p \_j n k s p a-2 k s a^{5} k s p a^{2} n^{2} r h o C r h o P h n p \_h n k s a$ 
So we now have numerator and denominator expressions for each coefficient (all denominators identical).

Now work on numerator and denominator separately, expressing as series in kspa (because kspa is small if kcpa is small and the particle is solid)

First do the first term of each series - these will be the terms that give the $\mathrm{n}>=2$ expressions

TnCC_ $=\left(A+B^{*} k s^{\prime} a^{\wedge} 2\right) /\left(C+D^{*} k s^{\prime} a^{\wedge} 2\right)$ where both $A$ and $C$ include factor of $(n-1)$.

This means that the case $n=1$ comes from $B / D$ and the cases $n>=2$ are $A / C$ 
and

TnCS_ $=\left(E+F k s^{\prime} a^{\wedge} 2\right) /\left(C+D^{*} k^{\prime} a^{\wedge} 2\right)$ etc.

$\begin{aligned}>\text { jnp_jnkspa } & :=\operatorname{subs}\left(x=k s p a, j n p_{\perp} \text { jnxx }\right) \\ \text { jnp_jnkspa } & :=\frac{n}{k s p a}-\frac{1}{2 n+3} k s p a-\frac{1}{(2 n+3)\left(4 n^{2}+16 n+15\right)} k s p a^{3}+\mathrm{O}\left(k s p a^{5}\right)\end{aligned}$

****************************************************************************************

$* * * * * * * * * * * * * * * * * * * * * * * * * * * *$

First work on TnCC_

$>$ numTnCC_Serkspa $:=\operatorname{simplify}(\operatorname{series}($ numTnCC_kspa, 8) $)$

numTnCC_Serkspa $:=-\frac{1}{2 n+3}\left(2 n k s a^{4} r h o P^{2}\left(2 k s a n^{3} h n p \_h n k s a+2 k s a n^{2} h n p \_h n k s a\right.\right.$

(24)

$\left.\left.-2 n^{4}-k s a n h n p \_h n k s a-2 n^{3}-3 k s a h n p \_h n k s a+n^{2}+3 n\right)\right) k s p a^{2}$

$-\frac{1}{(2 n+3)\left(4 n^{2}+16 n+15\right)}\left(n k s a^{2}\right.$ rhoP $\left(8 k s a^{3} n^{3}\right.$ rhoChnp_hnksa

$-12 k s a^{3} n^{3}$ rhoP hnp_hnksa $+36 k s a^{3} n^{2}$ rhoC hnp_hnksa $-40 k s a^{3} n^{2}$ rhoP hnp_hnksa

$+8 \mathrm{ksa}^{2} \mathrm{n}^{4} \mathrm{rhoC}+12 \mathrm{ksa}^{2} \mathrm{n}^{4} \mathrm{rhoP}+46 \mathrm{ksa}^{3} \mathrm{n}$ rhoChnp_hnksa

$-38 \mathrm{ksa}^{3} n$ rhoP hnp_hnksa $+20 \mathrm{ksa}^{2} \mathrm{n}^{3} \mathrm{rhoC}+40 \mathrm{ksa} \mathrm{a}^{2} \mathrm{n}^{3} \mathrm{rhoP}$

$-24 k s a n^{3}$ rhoChnp_hnksa $+15 \mathrm{ksa}^{3}$ rhoChnp_hnksa - $15 \mathrm{ksa}^{3}$ rhoP hnp_hnksa

$-26 k s a^{2} n^{2} r h o C+38 k s a^{2} n^{2}$ rhoP $-72 k s a n^{2}$ rhoChnp_hnksa $+24 n^{4}$ rhoC

$-77 \mathrm{ksa}^{2} \mathrm{n}$ rhoC $+15 \mathrm{ksa}^{2} \mathrm{n}$ rhoP $+6 \mathrm{ksan}$ rhoC hnp_hnksa $+72 n^{3}$ rhoC $-30 \mathrm{ksa}^{2}$ rhoC

+90 ksarhoChnp_hnksa $\left.\left.-6 n^{2} r h o C-90 n r h o C\right)\right) k s p a^{4}+\mathrm{O}\left(k s p a^{6}\right)$

$>$ denTnCC_Serkspa $:=\operatorname{simplify}(\operatorname{series}(\operatorname{denTnCC}, k s p a, 8)) \#$

denTnCC_Serkspa $:=-\frac{1}{2 n+3}\left(2 k s a^{4} r h o P^{2}\left(2 k s a n^{4} h n p \_h n k s a+4 k s a n^{3} h n p \_h n k s a+2 n^{5}\right.\right.$

$+k s a n^{2} h n p \_h n k s a+6 n^{4}-4 k s a n h n p \_h n k s a+5 n^{3}-3 k s a h n p \_h n k s a-3 n^{2}-7 n$

$-3)) k s p a^{2}+\frac{1}{(2 n+3)\left(4 n^{2}+16 n+15\right)}\left(k s a^{2} r h o P\left(8 k s a^{3} n^{4} r h o C h n p \_h n k s a\right.\right.$

$+12 \mathrm{ksa}^{3} n^{4}$ rhoP hnp_hnksa $+36 \mathrm{ksa}^{3} n^{3}$ rhoChnp_hnksa $+52 \mathrm{ksa} a^{3} n^{3}$ rhoP hnp_hnksa

$-8 k s a^{2} n^{5}$ rhoC $+12 k s a^{2} n^{5} r h o P+46 k s a^{3} n^{2}$ rhoC hnp_hnksa

$+78 k s a^{3} n^{2}$ rhoP hnp_hnksa $-60 k s a^{2} n^{4}$ rhoC $+64 k s a^{2} n^{4}$ rhoP

$+24 \mathrm{ksan}^{4}$ rhoChnp_hnksa $+15 \mathrm{ksa}^{3} \mathrm{n}$ rhoC hnp_hnksa $+53 \mathrm{ksa}^{3} \mathrm{n}$ rhoP hnp_hnksa

$-178 \mathrm{ksa}^{2} n^{3}$ rhoC $+130 \mathrm{ksa}^{2} n^{3}$ rhoP $+96 k s a n^{3}$ rhoChnp_hnksa $+24 n^{5}$ rhoC

$+15 k s a^{3}$ rhoP hnp_hnksa $-261 \mathrm{ksa}^{2} n^{2} \mathrm{rhoC}+131 \mathrm{ksa}^{2} \mathrm{n}^{2} \mathrm{rhoP}$

$+66 \mathrm{ksan}^{2}$ rhoChnp_hnksa $+120 n^{4} \mathrm{rhoC}-183 \mathrm{ksa}^{2} \mathrm{n}$ rhoC $+68 \mathrm{ksa}^{2} \mathrm{n}$ rhoP

-96 ksan rhoChnp_hnksa $+162 n^{3}$ rhoC $-45 \mathrm{ksa}^{2} \mathrm{rhoC}+15 \mathrm{ksa}^{2} \mathrm{rhoP}$

-90 ksa rhoC hnp_hnksa $\left.\left.-30 n^{2} r h o C-186 n r h o C-90 r h o C\right)\right) k s p a^{4}+\mathrm{O}\left(k s p a^{6}\right)$

Check the case $n=1$ to make sure both numerator and denominator are defined - then comment this out to retain general $\mathrm{n}$ for the overall solution.

$[>\# n:=1$ 


$$
\begin{aligned}
& >\text { \#numTnCC_Serkspa \# Confirms that only the second term in the series remains and the first term } \\
& \text { numTnCC_kspa2 }:=-\frac{2 k s a^{4} n r h o P^{2}\left(2 n^{3}+2 n^{2}-n-3\right)\left(k s a h n p \_h n k s a-n\right)}{2 n+3} \\
& \text { numTnCC_kspa2facs }:=-2 k s a^{4} n r h o P^{2}(n-1)\left(2 n^{2}+4 n+3\right) \\
& -\frac{2 k s a^{4} r h o P^{2}\left(2 n^{5}+6 n^{4}+5 n^{3}-3 n^{2}-7 n-3\right)}{2 n+3} \\
& \text { denTnCC_kspa2facs }:=-2(n-1)(n+1)\left(2 n^{2}+4 n+3\right)
\end{aligned}
$$

************************This (above) is the expression for the first term in the denominator for all coefficients

Combining these gives the expression for $\mathrm{n}>=2$

$$
\begin{aligned}
>T n C C_{-} n G E 2:= & \frac{n \cdot\left(k s a \cdot h n p \_h n k s a-n\right)}{(n+1) \cdot\left(k s a \cdot h n p \_h n k s a+(n+1)\right)} \\
& T n C C_{-} n G E 2:=\frac{n\left(k s a h n p \_h n k s a-n\right)}{(n+1)\left(k s a h n p \_h n k s a+n+1\right)}
\end{aligned}
$$

ENow the second term of each series - these are the terms that give the $\mathrm{n}=1$ expression

$>$ numTnCC_kspa4 $:=\operatorname{simplify}($ coeff (numTnCC_Serkspa, kspa,4) ) \#this is expression " $B$ " as written in the text above

numTnCC_kspa4 $:=-\frac{1}{(2 n+3)\left(4 n^{2}+16 n+15\right)}\left(n k s a^{2} r h o P\left(8 k s a^{3} n^{3}\right.\right.$ rhoChnp_hnksa

$-12 \mathrm{ksa}^{3} n^{3}$ rhoP hnp_hnksa $+36 \mathrm{ksa}^{3} n^{2}$ rhoC hnp_hnksa $-40 \mathrm{ksa} \mathrm{n}^{3} n^{2}$ rhoP hnp_hnksa

$+8 k s a^{2} n^{4} r h o C+12 k s a^{2} n^{4} r h o P+46 k s a^{3} n$ rhoChnp_hnksa

$-38 \mathrm{ksa}^{3} n$ rhoP hnp_hnksa $+20 \mathrm{ksa}^{2} \mathrm{n}^{3} \mathrm{rhoC}+40 \mathrm{ksa} \mathrm{a}^{2} \mathrm{n}^{3} \mathrm{rhoP}$

$-24 \mathrm{ksan}^{3}$ rhoChnp_hnksa $+15 \mathrm{ksa}^{3}$ rhoC hnp_hnksa $-15 \mathrm{ksa}^{3}$ rhoP hnp_hnksa

$-26 k s a^{2} n^{2} r h o C+38 k s a^{2} n^{2} r h o P-72 k s a n^{2} r h o C h n p \_h n k s a+24 n^{4}$ rhoC 
$-77 \mathrm{ksa}^{2} \mathrm{n}$ rhoC $+15 \mathrm{ksa}^{2} \mathrm{n} \mathrm{rhoP}+6 \mathrm{ksan}$ rhoC hnp_hnksa $+72 n^{3} \mathrm{rhoC}-30 \mathrm{ksa}^{2} \mathrm{rhoC}$

+ 90 ksa rhoChnp_hnksa $-6 n^{2}$ rhoC $-90 n$ rhoC) )

SSee what factors this expression - expecting (hnp_hnksa-1) but it may only factorise for the case $\mathrm{n}=1$ $>$ numTnCC_kspa4facs

$$
:=\text { collect }\left(\text { simplify }\left(\text { expand }\left(\frac{n u m T n C C \_k s p a 4 \cdot(2 \cdot n+3) \cdot\left(4 \cdot n^{2}+16 \cdot n+15\right)}{n \cdot k s a^{2} \cdot r h o P}\right)\right)\right. \text {, }
$$

hnp_hnksa)

numTnCC_kspa4facs $:=\left(-8 \mathrm{ksa}^{3} n^{3} \mathrm{rhoC}+12 \mathrm{ksa} a^{3} n^{3} \mathrm{rhoP}-36 \mathrm{ksa} a^{3} n^{2} \mathrm{rhoC}\right.$

$+40 k s a^{3} n^{2} r h o P-46 k s a^{3} n r h o C+38 k s a^{3} n r h o P+24 k s a n^{3} r h o C-15 k s a^{3} r h o C$

$\left.+15 \mathrm{ksa}^{3} \mathrm{rhoP}+72 \mathrm{ksan^{2 }} \mathrm{rhoC}-6 \mathrm{ksan} r h o C-90 \mathrm{ksarhoC}\right) \mathrm{hnp} \_$hnksa

$-8 k s a^{2} n^{4} r h o C-12 k s a^{2} n^{4} r h o P-20 k s a^{2} n^{3} r h o C-40 k s a^{2} n^{3} r h o P+26 k s a^{2} n^{2} r h o C$

$-38 k s a^{2} n^{2}$ rhoP $-24 n^{4}$ rhoC $+77 k s a^{2} n$ rhoC $-15 k s a^{2} n$ rhoP $-72 n^{3}$ rhoC

$+30 \mathrm{ksa}^{2} \mathrm{rhoC}+6 n^{2}$ rhoC $+90 n$ rhoC

Note that $\left(4 n^{\wedge} 2+16 n+15\right)$ factorises to $(2 n+3)(2 n+5)$

$>$ denTnCC_kspa4 $:=\operatorname{simplify}(\operatorname{coeff}(\operatorname{denTnCC}$ SSerkspa, kspa, 4) )

\#this is the expression " $D$ " as written in the text above

denTnCC_kspa4 $:=\frac{1}{(2 n+3)\left(4 n^{2}+16 n+15\right)}\left(k s a^{2}\right.$ rhoP $\left(8 k s a^{3} n^{4}\right.$ rhoChnp_hnksa

$+12 \mathrm{ksa}^{3} n^{4}$ rhoP hnp_hnksa $+36 \mathrm{ksa}^{3} n^{3}$ rhoChnp_hnksa $+52 \mathrm{ksa}^{3} \mathrm{n}^{3}$ rhoP hnp_hnksa

$-8 k s a^{2} n^{5}$ rhoC $+12 k s a^{2} n^{5} r h o P+46 k s a^{3} n^{2}$ rhoC hnp_hnksa

$+78 k s a^{3} n^{2}$ rhoP hnp_hnksa $-60 k s a^{2} n^{4}$ rhoC $+64 k s a^{2} n^{4}$ rhoP

$+24 \mathrm{ksan}^{4}$ rhoC hnp_hnksa $+15 \mathrm{ksa}^{3} \mathrm{n}$ rhoChnp_hnksa $+53 \mathrm{ksa}^{3} \mathrm{n}$ rhoP hnp_hnksa

$-178 \mathrm{ksa}^{2} n^{3}$ rhoC $+130 \mathrm{ksa}^{2} n^{3}$ rhoP $+96 \mathrm{ksan} n^{3}$ rhoChnp_hnksa $+24 n^{5}$ rhoC

$+15 k s a^{3}$ rhoP hnp_hnksa $-261 \mathrm{ksa}^{2} n^{2} \mathrm{rhoC}+131 k s a^{2} n^{2}$ rhoP

$+66 \mathrm{ksan}^{2}$ rhoChnp_hnksa $+120 n^{4} \mathrm{rhoC}-183 \mathrm{ksa}^{2} n \mathrm{rhoC}+68 \mathrm{ksa}^{2} n \mathrm{rhoP}$

-96 ksa $n$ rhoC hnp_hnksa $+162 n^{3}$ rhoC $-45 \mathrm{ksa}^{2} \mathrm{rhoC}+15 \mathrm{ksa}^{2} \mathrm{rhoP}$

-90 ksa rhoC hnp_hnksa $-30 n^{2}$ rhoC $-186 n$ rhoC -90 rhoC) )

$>$ denTnCC_kspa4facs

$$
:=\operatorname{collect}\left(\operatorname{simplify}\left(\operatorname{expand}\left(\frac{\operatorname{denTnCC} k \operatorname{spa} 4 \cdot(2 \cdot n+3) \cdot\left(4 \cdot n^{2}+16 \cdot n+15\right)}{k s a^{2} \cdot r h o P}\right)\right),\right.
$$

$\left.h n p \_h n k s a\right)$

denTnCC_kspa4facs $:=\left(8 \mathrm{ksa}^{3} n^{4}\right.$ rhoC $+12 \mathrm{ksa} a^{3} n^{4}$ rhoP $+36 \mathrm{ksa}^{3} n^{3} \mathrm{rhoC}+52 \mathrm{ksa} \mathrm{n}^{3} \mathrm{rhoP}$

$+46 k s a^{3} n^{2} r h o C+78 k s a^{3} n^{2} r h o P+24 k s a n^{4} r h o C+15 k s a^{3} n r h o C+53 k s a^{3} n r h o P$

$+96 \mathrm{ksan}^{3} \mathrm{rhoC}+15 \mathrm{ksa}^{3} \mathrm{rhoP}+66 \mathrm{ksan} \mathrm{n}^{2} \mathrm{rhoC}-96 \mathrm{ksan}$ rhoC $\left.-90 \mathrm{ksarhoC}\right)$

hnp_hnksa $-8 k s a^{2} n^{5} r h o C+12 k s a^{2} n^{5} r h o P-60 k s a^{2} n^{4} r h o C+64 k s a^{2} n^{4}$ rhoP

$-178 k s a^{2} n^{3} r h o C+130 k s a^{2} n^{3} r h o P+24 n^{5} r h o C-261 k s a^{2} n^{2} r h o C$ 
$+131 k s a^{2} n^{2} r h o P+120 n^{4} r h o C-183 k s a^{2} n r h o C+68 k s a^{2} n r h o P+162 n^{3} r h o C$

$-45 \mathrm{ksa}^{2} \mathrm{rhoC}+15 \mathrm{ksa}^{2}$ rhoP $-30 n^{2}$ rhoC $-186 n$ rhoC $-90 \mathrm{rhoC}$

Try and simplify the parts of these expressions. First the second term of the numerator series, starting with the terms in hnp_hnksa

We know that there is a factor of (ksa*hnp_hnksa-n) and a factor (rhoHat-1) in the $\mathrm{n}=1$ solution So collect terms in rhoC(ksa*hnp_hn) and then rhoP(ksa*hnp_hn) and those in $\mathrm{n}^{*} \mathrm{rhoC}$ and $\mathrm{n} * \mathrm{rhoP}$. Use the matching terms in rhoP to collect (rhoP-rhoC)(ksahnp_hn-n) and leave remaining terms just in rhoC. These remaining terms turn out to have factors ( $n-1)$ which means they vanish for $n=1$. This simplification was completed by hand. Check it matches what was obtained above

$>$ numTnCC_kspa4facsSimpl $:=\left(12 \cdot n^{3}+40 \cdot n^{2}+38 \cdot n+15\right) \cdot(r h o P-r h o C) \cdot k s a^{2} \cdot(k s a$ $\left.\cdot h n p \_h n k s a-n\right)+4 \cdot n \cdot(n-1) \cdot(n+2) \cdot r h o C \cdot k s a^{2} \cdot\left(k s a \cdot h n p \_h n k s a-n\right)+6 \cdot(n-1) \cdot(2$ $\cdot n+\overline{3}) \cdot(2 \cdot n+5) \cdot r h o C \cdot\left(k s a \cdot h n p \_h n k s a-n\right)-2 \cdot(n-1) \cdot(\overline{2} \cdot n+1) \cdot(2 \cdot n+3) \cdot(2 \cdot n$ $+5) \cdot r h o C \cdot k s a^{2}$

numTnCC_kspa4facsSimpl $:=\left(12 n^{3}+40 n^{2}+38 n+15\right)($ rhoP $-r h o C) k s a^{2}\left(k s a h n p \_h n k s a\right.$

$-n)+4 n(n-1)(n+2)$ rhoC ksa $^{2}$ (ksa hnp_hnksa-n)+6(n-1) $(2 n+3)(2 n$

$+5)$ rhoC (ksahnp_hnksa $-n)-2(n-1)(2 n+1)(2 n+3)(2 n+5) r h o C k s a^{2}$

****************This (above) is the simplified result for the second term in numerator of TnCC

(when combined with the factors in line 32)

$>$ expand(numTnCC_kspa4facsSimpl-numTnCC_kspa4facs)

\# confirm that this is zero, therefore the simplification is correct.

Now try and simplify the second term in the series in kspa in the denominator denTnCC_kspa4facs. Based on some analytical simplifications, and by comparing with the $n=1$ known result, guessing that the remaining terms will be of the form

$(2 \mathrm{n}+1) *$ rhoC*(ksa*hnp/hn-n $)+(\mathrm{n}+1) *($ rhoP-rhoC $) *\left(\mathrm{ksa}^{*} \mathrm{hnp} / \mathrm{hn}+(\mathrm{n}+1)\right)$






$$
\begin{aligned}
& \left.(-n)-\operatorname{rhoC} \cdot\left(24 \cdot n^{4}+96 \cdot n^{3}+66 \cdot n^{2}-96 \cdot n-90\right) \cdot\left(k s a \cdot h n p \_h n k s a-n\right)\right) \\
& \text { denRhoCLeft } 2:=24 \mathrm{ksa}^{2} n^{5} \text { rhoC }+92 \mathrm{ksa}^{2} n^{4} \mathrm{rhoC}+76 \mathrm{ksa}^{2} \mathrm{n}^{3} \mathrm{rhoC}+48 \mathrm{n}^{5} \mathrm{rhoC} \\
& -62 k s a^{2} n^{2} r h o C+216 n^{4} r h o C-100 k s a^{2} n r h o C+228 n^{3} r h o C-30 k s a^{2} r h o C \\
& -126 n^{2} \text { rhoC }-276 n \text { rhoC }-90 \text { rhoC } \\
& >\operatorname{denRhoCLeft3}:=\operatorname{collect}\left(\operatorname{simplify}\left(\operatorname{expand}\left(\frac{\text { denRhoCLeft } 2}{(n-1)}\right)\right), k s a\right) \\
& \text { denRhoCLeft } 3:=2\left(12 n^{4}+58 n^{3}+96 n^{2}+65 n+15\right) r h o C k s a^{2}+2\left(24 n^{4}+132 n^{3}\right. \\
& \left.+246 n^{2}+183 n+45\right) \text { rhoC }
\end{aligned}
$$

So we have obtained a simpified result for the second term of the series in the denominator. Because these remaining terms have a factor (n-1) and we have a reasonably short expression for them. Now reconstruct it (with factorisations done manually) and check that the simplified form is correct.

Check by subtracting each part of the simplified expression from the full result (with factors removed) sequentially

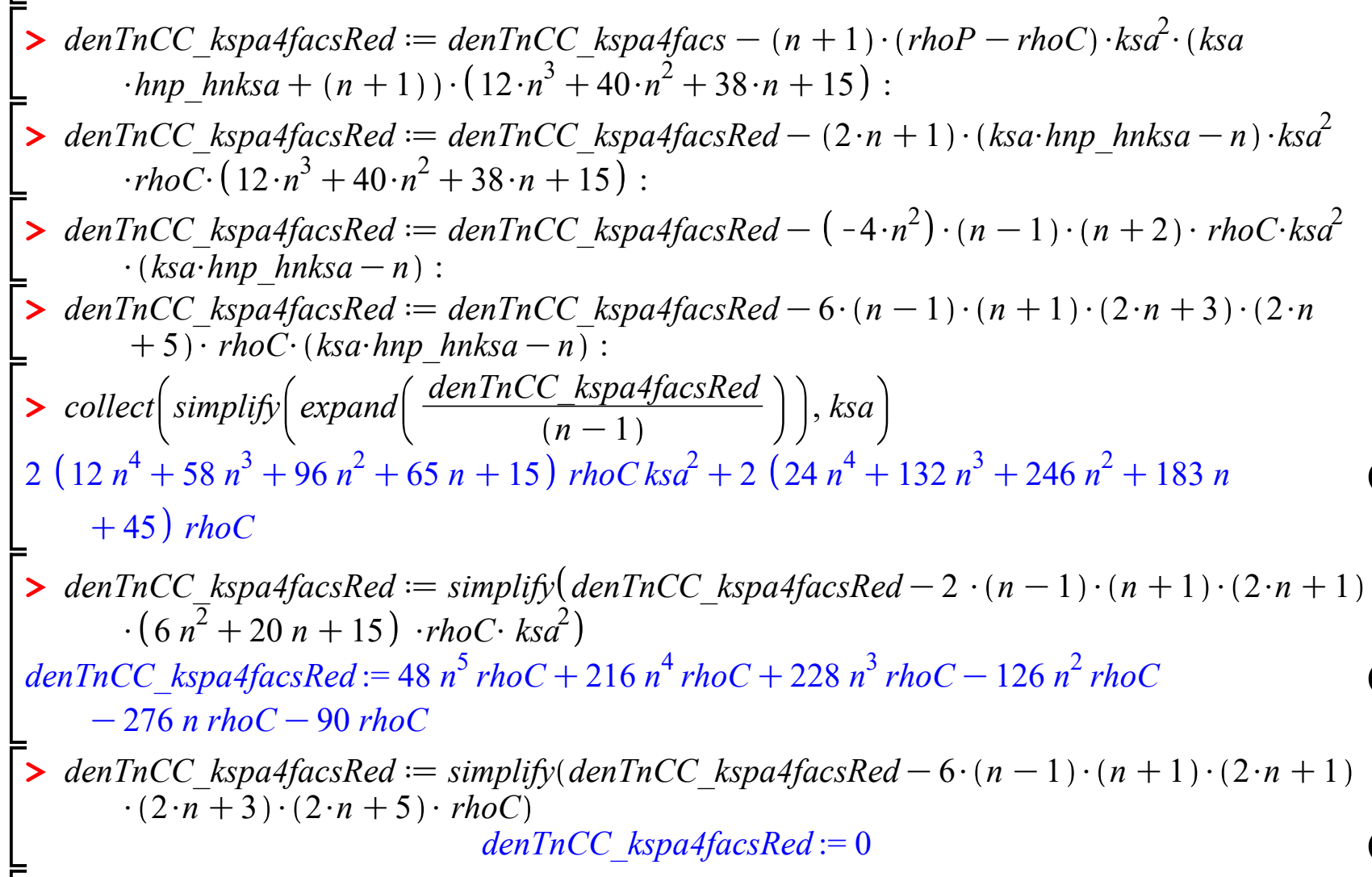

Therefore these set of simplified expressions agree with the original second term of series in deminator. So the set of expressions just above allow the construction of the term

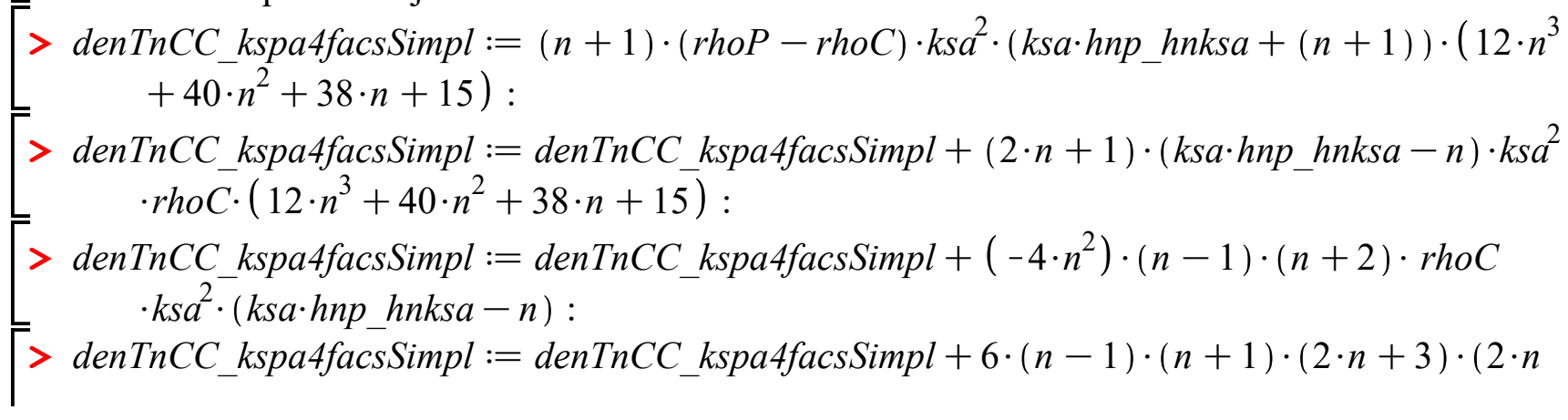




$$
\begin{aligned}
& L \quad+5) \cdot \operatorname{rhoC} \cdot\left(k s a \cdot h n p \_h n k s a-n\right): \\
& \text { > denTnCC_kspa4facsSimpl }:=\text { denTnCC_kspa4facsSimpl }+2 \cdot(n-1) \cdot(n+1) \cdot(2 \cdot n+1) \cdot\left(6 n^{2}\right. \\
& +20 n+15) \cdot r h o C \cdot k s a^{2}: \\
& >\text { denTnCC_kspa4facsSimpl }:=\text { denTnCC_kspa4facsSimpl }+6 \cdot(n-1) \cdot(n+1) \cdot(2 \cdot n+1) \cdot(2 \cdot n \\
& +3) \cdot(2 \cdot n+5) \cdot \text { rhoC: }
\end{aligned}
$$

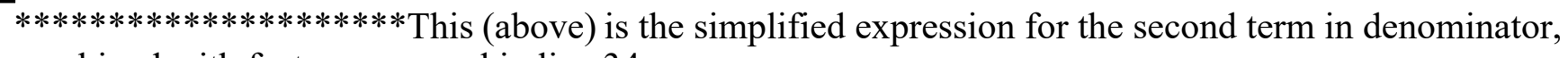

$$
\begin{aligned}
& \text { combined with factors removed in line } 34 \text {. } \\
& >\operatorname{simplify}(\operatorname{denTnCC} \text { _kspa4facs - denTnCC_kspa4facsSimpl) } \\
& \text { \# this is zero, so the simplification is correct. } \\
& 0 \\
& (n+1)(r h o P-r h o C) k s a^{2}\left(k s a h n p \_h n k s a+n+1\right)\left(12 n^{3}+40 n^{2}+38 n+15\right)+(2 n \\
& +1)\left(k s a h n p \_h n k s a-n\right) k s a^{2} \text { rhoC }\left(12 n^{3}+40 n^{2}+38 n+15\right)-4 n^{2}(n-1)(n \\
& +2) \text { rhoC ksa }{ }^{2}\left(k s a h n p \_h n k s a-n\right)+6(n-1)(n+1)(2 n+3)(2 n \\
& +5) \text { rhoC }(\text { ksa hnp_hnksa }-n)+2(n-1)(n+1)(2 n+1)\left(6 n^{2}+20 n\right. \\
& +15) r h o C k s a^{2}+6(n-1)(n+1)(2 n+1)(2 n+3)(2 n+5) r h o C
\end{aligned}
$$

Now work on the other coefficients. First TnCS_(scaled) numerator only (since denominator is identical)

$$
\begin{aligned}
& >\text { numTnCS_Serkspa }:=\operatorname{simplify}(\operatorname{series}(\text { numTnCS_, kspa, 8) ) } \\
& \text { numTnCS_Serkspa }:=\frac{2 k s a^{4} \operatorname{rhoP}^{2}\left(4 n^{4}+6 n^{3}-7 n-3\right)}{2 n+3} k s p a^{2} \\
& -\frac{1}{(2 n+3)\left(4 n^{2}+16 n+15\right)}\left(k s a ^ { 2 } r h o P \left(24 k s a^{2} n^{4} r h o P-24 k s a^{2} n^{3} r h o C\right.\right. \\
& +92 k s a^{2} n^{3} r h o P-108 k s a^{2} n^{2} r h o C+116 k s a^{2} n^{2} r h o P+48 n^{4} r h o C-138 k s a^{2} n r h o C \\
& +68 k s a^{2} n r h o P+168 n^{3} r h o C-45 k s a^{2} r h o C+15 k s a^{2} r h o P+60 n^{2} r h o C-186 n r h o C \\
& -90 r h o C)) k s p a^{4}+\mathrm{O}\left(k s p a^{6}\right) \\
& >\text { numTnCS_kspa2 }:=\operatorname{simplify}((\operatorname{coeff}(\text { numTnCS_Serkspa,kspa, 2) ) ) } \\
& \text { numTnCS_kspa2 }:=\frac{2 k s a^{4} r h o \overline{P^{2}}\left(4 n^{4}+6 n^{3}-7 n-3\right)}{2 n+3} \\
& >\text { numTnCS_kspa4 }:=\operatorname{simplify}(\operatorname{coeff}(\text { numTnCS_Serkspa,kspa,4) ) } \\
& \text { \# this is expression " } F \text { " as written in the text above - second term in numerator } \\
& \text { numTnCS_kspa4 }:=-\frac{1}{(2 n+3)\left(4 n^{2}+16 n+15\right)}\left(k s a ^ { 2 } \text { rhoP } \left(24 k s a^{2} n^{4}\right.\right. \text { rhoP } \\
& -24 k s a^{2} n^{3} r h o C+92 k s a^{2} n^{3} r h o P-108 k s a^{2} n^{2} r h o C+116 k s a^{2} n^{2} r h o P+48 n^{4} r h o C
\end{aligned}
$$




$$
\begin{aligned}
& -138 k s a^{2} n r h o C+68 k s a^{2} n r h o P+168 n^{3} r h o C-45 k s a^{2} r h o C+15 k s a^{2} r h o P \\
& +60 n^{2} \text { rhoC }-186 n \text { rhoC }-90 \text { rhoC) ) } \\
& \text { Eremove factors } \\
& >\text { numTnCS_kspa4facs } \\
& :=\text { collect }\left(\operatorname{simplify}\left(\operatorname{expand}\left(\frac{n u m T n C S \_k s p a 4 \cdot(2 \cdot n+3) \cdot\left(4 \cdot n^{2}+16 \cdot n+15\right)}{k s a^{2} \cdot r h o P}\right)\right), r h o P\right) \\
& \text { numTnCS_kspa4facs }:=\left(-24 k s a^{2} n^{4}-92 k s a^{2} n^{3}-116 k s a^{2} n^{2}-68 k s a^{2} n-15 k s a^{2}\right) r h o P \\
& +24 k s a^{2} n^{3} r h o C+108 k s a^{2} n^{2} r h o C-48 n^{4} r h o C+138 k s a^{2} n r h o C-168 n^{3} \text { rhoC } \\
& +45 \mathrm{ksa}^{2} \mathrm{rhoC}-60 n^{2} \mathrm{rhoC}+186 \mathrm{n} r h o \mathrm{C}+90 \mathrm{rhoC}
\end{aligned}
$$

Collect terms to result in a (rhoP-rhoC) expression (which appears in the $n=1$ coefficient) and remaining terms with a factor of (n-1). Manual factorisation.

$$
\begin{aligned}
& >\text { numTnCS_kspa4facsSimpl }:=-(\text { rhoP }-r h o C) \cdot k s a^{2} \cdot(2 \cdot n+1) \cdot\left(12 \cdot n^{3}+40 \cdot n^{2}+38 \cdot n+15\right) \\
& \quad-2 \cdot r h o C \cdot k s a^{2} \cdot(n-1) \cdot(2 \cdot n+1) \cdot\left(6 \cdot n^{2}+20 \cdot n+15\right)-6 \cdot r h o C \cdot(n-1) \cdot(2 \cdot n+1) \cdot(2 \\
& \quad \cdot n+3) \cdot(2 \cdot n+5) \\
& \text { num TnCS_kspa4facsSimpl }:=-(r h o P-r h o C) k s a^{2}(2 n+1)\left(12 n^{3}+40 n^{2}+38 n+15\right) \\
& \quad-2 \text { rhoC ksa }(n-1)(2 n+1)\left(6 n^{2}+20 n+15\right)-6 r h o C(n-1)(2 n+1)(2 n \\
& \quad+3)(2 n+5)
\end{aligned}
$$

**************This is the simplified expression for the second term in the denominator of TnCS (combined with factors in line 49)

$>\operatorname{simplify}(\operatorname{expand}($ numTnCS_kspa4facsSimpl - numTnCS_kspa4facs) )

\#check that the simplified version agrees with the original

0

F**

$* * * * * * * * * * * * * * * * * * * * * * * * * * * * * * * * * * * * *$

Now work on numerator of TnSC_ (scaled). Denominator is identical to TnCC_

$>$ numTnSC_Serkspa $:=\operatorname{simplify}(\operatorname{series}($ numTnSC_, kspa, 8))

numTnSC_Serkspa $:=-\frac{1}{2 n+3}\left(2 n(n+1) k s a^{5}\right.$ rhoP $P^{2}\left(2 n^{3} h n p \_h n k s a-2 n^{3} j n p \_j n k s a\right.$

$+2 n^{2} h n p \_h n k s a-2 n^{2} j n p \_j n k s a-n h n p \_h n k s a+n j n p \_j n k s a-3 h n p \_h n k s a$

$\left.\left.+3 j n p \_j n k s a\right)\right) k s p a^{2}+\frac{1}{(2 n+3)\left(4 n^{2}+16 n+15\right)}(n(n$

$+1) k_{s a}^{3}$ rhoP $\left(12 k s a^{2} n^{3}\right.$ rhoP hnp_hnksa - $12 k s a^{2} n^{3}$ rhoP jnp_jnksa

$-12 \mathrm{ksa}^{2} n^{2}$ rhoC hnp_hnksa $+12 \mathrm{ksa}^{2} \mathrm{n}^{2}$ rhoCjnp_jnksa $+40 \mathrm{ksa}^{2} \mathrm{n}^{2}$ rhoP hnp_hnksa

$-40 \mathrm{ksa}^{2} \mathrm{n}^{2}$ rhoP jnp_jnksa $-48 \mathrm{ksa}^{2} \mathrm{n}$ rhoC hnp_hnksa $+48 \mathrm{ksa}^{2} \mathrm{n}$ rhoCjnp_jnksa

$+38 \mathrm{ksa}^{2} n$ rhoP hnp_hnksa $-38 \mathrm{ksa}^{2} n$ rhoP jnp_jnksa $+24 n^{3}$ rhoC hnp_hnksa

$-24 n^{3}$ rhoCjnp_jnksa $-45 \mathrm{ksa}^{2}$ rhoC hnp_hnksa $+45 \mathrm{ksa} a^{2}$ rhoCjnp_jnksa

+ $15 \mathrm{ksa}^{2}$ rhoP hnp_hnksa - $15 \mathrm{ksa}^{2}$ rhoP jnp_jnksa $+72 n^{2}$ rhoC hnp_hnksa

$-72 n^{2}$ rhoCjnp_jnksa - $6 n$ rhoC hnp_hnksa $+6 n$ rhoCjnp_jnksa -90 rhoC hnp_hnksa

+ 90 rhoCjnp_jnksa) ) $k s p a^{4}+\mathrm{O}\left(k s p a^{6}\right)$

$>$ numTnSC_kspa2 $:=\operatorname{simplify}((\operatorname{coeff}($ numTnSC_Serkspa, kspa, 2 ) ) 


$$
\begin{aligned}
& \text { numTnSC_kspa2 }:=-\frac{1}{2 n+3}\left(2 n ( n + 1 ) k s a ^ { 5 } r h o P ^ { 2 } \left(2 n^{3} h n p \_h n k s a-2 n^{3} j n p \_j n k s a\right.\right. \\
& +2 n^{2} h n p \_h n k s a-2 n^{2} j n p \_j n k s a-n h n p \_h n k s a+n j n p \_j n k s a-3 h n p \_h n k s a \\
& +3 \text { jnp_jnksa)) } \\
& \text { > numTnSC_kspa2Simpl1 }:=\operatorname{simplify}\left(\operatorname { e x p a n d } \left(\operatorname { s u b s } \left(j n p \_j n k s a=h n p \_h n k s a\right.\right.\right. \\
& -\frac{I}{k s a^{2} \cdot j n k s a \cdot h n k s a}, \text { numTnSC_kspa2)))\#use the Heine relation to simplify } \\
& \text { numTnSC_kspa2Simpll }:=-\frac{2 \operatorname{I} n k s a^{3} r h o P^{2}\left(2 n^{4}+4 n^{3}+n^{2}-4 n-3\right)}{(2 n+3) j n k s a h n k s a}
\end{aligned}
$$

$* * * * * * * * * * * * * * * * * * *$ This (above) is the simplified expression for the first term in the numerator of LnSC

$>$ numTnSC_kspa4 $:=\operatorname{simplify}((\operatorname{coeff}($ numTnSC_Serkspa,kspa, 4 ) )

numTnSC_kspa4 $:=\frac{1}{(2 n+3)\left(4 n^{2}+16 n+15\right)}(n(n$

$+1) k s a^{3}$ rhoP $\left(12 k s a^{2} n^{3}\right.$ rhoP hnp_hnksa - $12 k s a^{2} n^{3}$ rhoP jnp_jnksa

$-12 \mathrm{ksa}^{2} n^{2}$ rhoC hnp_hnksa $+12 \mathrm{ksa}^{2} n^{2}$ rhoCjnp_jnksa $+40 \mathrm{ksa}^{2} n^{2}$ rhoP hnp_hnksa

$-40 \mathrm{ksa}^{2} \mathrm{n}^{2}$ rhoP jnp_jnksa $-48 \mathrm{ksa}^{2} \mathrm{n}$ rhoC hnp_hnksa $+48 \mathrm{ksa}^{2} \mathrm{n}$ rhoCjnp_jnksa

$+38 \mathrm{ksa}^{2} n$ rhoP hnp_hnksa $-38 \mathrm{ksa}^{2} n$ rhoP jnp_jnksa $+24 n^{3}$ rhoC hnp_hnksa

$-24 n^{3}$ rhoCjnp_jnksa $-45 \mathrm{ksa}^{2}$ rhoC hnp_hnksa $+45 \mathrm{ksa} a^{2}$ rhoCjnp_jnksa

+ $15 \mathrm{ksa}^{2}$ rhoP hnp_hnksa - $15 \mathrm{ksa}^{2}$ rhoP jnp_jnksa $+72 \mathrm{n}^{2}$ rhoC hnp_hnksa

$-72 n^{2}$ rhoCjnp_jnksa $-6 n$ rhoC hnp_hnksa $+6 n$ rhoCjnp_jnksa -90 rhoC hnp_hnksa

+90 rhoCjnp_jnksa))

$>$ numTnSC_kspa4facs

$$
:=\left(\operatorname{simplify}\left(\operatorname{expand}\left(\frac{n u m T n S C \_k s p a 4 \cdot(2 \cdot n+3) \cdot\left(4 \cdot n^{2}+16 \cdot n+15\right)}{k s a^{2} \cdot r h o P}\right)\right)\right)
$$

numTnSC_kspa4facs $:=12 \mathrm{ksa}^{3} n^{5}$ rhoP hnp_hnksa $-12 \mathrm{ksa} \mathrm{n}^{3} \mathrm{rhoP}^{5} \mathrm{jn}$ junksa

$-12 \mathrm{ksa}^{3} n^{4}$ rhoC hnp_hnksa $+12 \mathrm{ksa}^{3} n^{4}$ rhoCjnp_jnksa $+52 \mathrm{ksa}^{3} n^{4}$ rhoP hnp_hnksa

$-52 \mathrm{ksa}^{3} n^{4}$ rhoP jnp_jnksa $-60 \mathrm{ksa} \mathrm{n}^{3} n^{3}$ rhoC hnp_hnksa $+60 \mathrm{ksa} a^{3} n^{3}$ rhoCjnp_jnksa

$+78 \mathrm{ksa}^{3} \mathrm{n}^{3}$ rhoP hnp_hnksa $-78 \mathrm{ksa}^{3} n^{3}$ rhoP jnp_jnksa $+24 \mathrm{ksa} n^{5}$ rhoChnp_hnksa

$-24 k s a n^{5}$ rhoCjnp_jnksa - $93 \mathrm{ksa}^{3} n^{2}$ rhoC hnp_hnksa $+93 \mathrm{ksa}^{3} n^{2}$ rhoCjnp_jnksa

$+53 \mathrm{ksa}^{3} n^{2}$ rhoP hnp_hnksa $-53 \mathrm{ksa}^{3} n^{2}$ rhoP jnp_jnksa $+96 \mathrm{ksan}{ }^{4}$ rhoChnp_hnksa

$-96 \mathrm{ksan}^{4}$ rhoCjnp_jnksa $-45 \mathrm{ksa}^{3} \mathrm{n}$ rhoC hnp_hnksa $+45 \mathrm{ksa}^{3} \mathrm{n}$ rhoCjnp_jnksa

$+15 \mathrm{ksa}^{3} n$ rhoP hnp_hnksa $-15 \mathrm{ksa}^{3} n$ rhoP jnp_jnksa $+66 \mathrm{ksa} n^{3}$ rhoC hnp_hnksa

- $66 \mathrm{ksan}^{3}$ rhoC jnp_jnksa - $96 \mathrm{ksa} n^{2}$ rhoC hnp_hnksa + $96 \mathrm{ksa} n^{2}$ rhoCjnp_jnksa

- 90 ksa n rhoC hnp_hnksa + 90 ksa $n$ rhoCjnp_jnksa

$>$ numTnSC_kspa4facsSimpl1 $:=$ collect $\left(\operatorname{simplify}\left(\right.\right.$ expand $\left(\operatorname{subs}\left(j n p \_j n k s a=h n p \_h n k s a\right.\right.$ 


$$
\begin{aligned}
& \left.\left.\left.\left.\quad-\frac{I}{k s a^{2} \cdot j n k s a \cdot h n k s a}, \text { numTnSC_kspa4facs }\right)\right)\right) \text {,rhoP }\right) \text { \#use the Heine relation to simplify } \\
& \text { numTnSC_kspa4facsSimpll }:= \\
& \quad \frac{\mathrm{I} n\left(12 k s a^{2} n^{4}+52 k s a^{2} n^{3}+78 k s a^{2} n^{2}+53 k s a^{2} n+15 k s a^{2}\right) r h o P}{k s a j n k s a h n k s a} \\
& +\frac{1}{k s a j n k s a h n k s a}\left(\mathrm { I } n \left(-12 k s a^{2} n^{3} r h o C-60 k s a^{2} n^{2} r h o C+24 n^{4} r h o C\right.\right. \\
& \left.\left.-93 k s a^{2} n \text { rhoC }+96 n^{3} \text { rhoC }-45 k s a^{2} \text { rhoC }+66 n^{2} r h o C-96 n \text { rhoC }-90 \text { rhoC }\right)\right)
\end{aligned}
$$

Now collect terms in (rhoP-rhoC) which appear in the (n-1) expression, and the rest should have a factor $(\mathrm{n}-1)$ and therefore vanish when $\mathrm{n}=1$. Simplified by hand

$$
\begin{aligned}
& >\text { numTnSC_kspa4facsSimpl }:=\frac{1}{k s a \cdot j n k s a \cdot h n k s a}\left(I \cdot n \cdot \left[( r h o P - r h o C ) \cdot k s a ^ { 2 } \cdot ( n + 1 ) \cdot \left(12 \cdot n^{3}\right.\right.\right. \\
& \left.\quad+40 \cdot n^{2}+38 \cdot n+15\right)+2 \cdot r h o C \cdot(n-1) \cdot(n+1) \cdot\left(\left(6 \cdot n^{2}+20 \cdot n+15\right) \cdot k s a^{2}+3 \cdot(2 n\right. \\
& \quad+3) \cdot(2 n+5))]) \\
& \text { numTnSC_kspa4facsSimpl }:=\frac{1}{k s a j n k s a h n k s a}\left(I n \left[( r h o P - r h o C ) k s a ^ { 2 } ( n + 1 ) \left(12 n^{3}\right.\right.\right. \\
& \left.\quad+40 n^{2}+38 n+15\right)+2 \operatorname{rhoC}(n-1)(n+1)\left(\left(6 n^{2}+20 n+15\right) k s a^{2}+3(2 n\right. \\
& \quad+3)(2 n+5))])
\end{aligned}
$$

********************This (above) is the simplified expression for the second term in the numerator Lof TnSC_, combined with factors in line 56.

$>$ expand (simplify ( (collect (expand $\left(k s a \cdot j n k s a \cdot h n k s a \cdot n u m T n S C \_k s p a 4 f a c s S i m p l-k s a \cdot j n k s a \cdot h n k s a\right.$ -numTnSC_kspa4facsSimpl1), rhoP) ) ) )

\#this is zero (but Maple won't simplify it to zero) so the simplification is correct

$$
\begin{aligned}
& {\left[12 \mathrm{In}^{5} \mathrm{ksa}^{2} \mathrm{rhoP}-12 \mathrm{In}^{4} \mathrm{ksa}^{2} \mathrm{rhoC}+52 \mathrm{I}^{4} \mathrm{ksa}^{2} \mathrm{rhoP}-60 \mathrm{I}^{3} \mathrm{ksa}^{2} \mathrm{rhoC}+78 \mathrm{I} n^{3} \mathrm{ksa}^{2} \mathrm{rhoP}\right.} \\
& +24 \mathrm{I} n^{5} r h o C-93 \mathrm{I}^{2} k s a^{2} r h o C+53 \mathrm{I}^{2} k s a^{2} r h o P+96 \mathrm{I} n^{4} r h o C-45 \mathrm{I} n k s a^{2} r h o C
\end{aligned}
$$

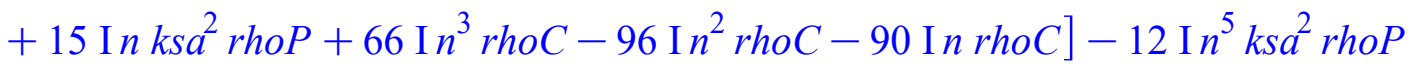

$$
\begin{aligned}
& +12 \mathrm{I}^{4} k s a^{2} r h o C-52 \mathrm{I}^{4} k s a^{2} r h o P+60 \mathrm{In}^{3} k s a^{2} r h o C-78 \mathrm{I} n^{3} k s a^{2} r h o P \\
& -24 \mathrm{I} n^{5} r h o C+93 \mathrm{I}^{2} k s a^{2} r h o C-53 \mathrm{I}^{2} k s a^{2} r h o P-96 \mathrm{I} n^{4} r h o C+45 \mathrm{I} n k s a^{2} \text { rhoC } \\
& -15 \mathrm{I} n k s a^{2} r h o P-66 \mathrm{I}^{3} r h o C+96 \mathrm{I} n^{2} r h o C+90 \mathrm{I} n r h o C \\
& \text { ****************************************************************************************** } \\
& * * * * * * * * * * * * * * * * * * * * * * * * * * * * *
\end{aligned}
$$

Now TnSS_numerator (denominator is identical)

$>$ numTnSS_Serkspa $:=\operatorname{simplify}(\operatorname{series}($ numTnSS_, kspa, 8) )

numTnSS_Serkspa $:=\frac{1}{2 n+3}\left(2 k s a^{4} r_{h o P^{2}}\left(2 k s a n^{4} j n p \_j n k s a+4 k s a n^{3} j n p \_j n k s a+2 n^{5}\right.\right.$

$\left.\left.+k s a n^{2} j n p \_j n k s a+6 n^{4}-4 k s a n j n p \_j n k s a+5 n^{3}-3 k s a j n p \_j n k s a-3 n^{2}-7 n-3\right)\right)$

$k s p a^{2}-\frac{1}{(2 n+3)\left(4 n^{2}+16 n+15\right)}\left(k s a^{2}\right.$ rhoP $\left(8 k s a^{3} n^{4}\right.$ rhoC jnp jnksa

$+12 \mathrm{ksa}^{3} n^{4}$ rhoP jnp_jnksa $+36 \mathrm{ksa}^{3} \mathrm{n}^{3}$ rhoCjnp_jnksa $+52 \mathrm{ksa} a^{3} n^{3}$ rhoP jnp_jnksa

$-8 k s a^{2} n^{5} r h o C+12 k s a^{2} n^{5} r h o P+46 k s a^{3} n^{2} r h o C j n p \_j n k s a$

$+78 k s a^{3} n^{2}$ rhoP jnp jnksa $-60 k s a^{2} n^{4} r h o C+64 k s a^{2} n^{4}$ rhoP 


$$
\begin{aligned}
& +24 \mathrm{ksan}^{4} \text { rhoCjnp_jnksa }+15 \mathrm{ksa}^{3} \text { n rhoCjnp_jnksa }+53 \mathrm{ksa} a^{3} n \text { rhoP jnp_jnksa } \\
& -178 k s a^{2} n^{3} \text { rhoC }+130 k s a^{2} n^{3} r h o P+96 k s a n^{3} \text { rhoCjnp jnksa }+24 n^{5} \text { rhoC } \\
& +15 k s a^{3} \text { rhoP jnp jnksa }-261 k s a^{2} n^{2} \text { rhoC }+131 k s a^{2} n^{2} r h o P \\
& +66 k s a n^{2} \text { rhoCjnp_jnksa }+120 n^{4} \text { rhoC }-183 k s a^{2} n \text { rhoC }+68 k s a^{2} n \text { rhoP } \\
& -96 k s a n \text { rhoC jnp jnksa }+162 n^{3} \text { rhoC }-45 k s a^{2} \text { rhoC }+15 k s a^{2} \text { rhoP } \\
& \left.\left.-90 \text { ksa rhoC jnp_jnksa }-30 n^{2} \text { rhoC }-186 n \text { rhoC }-90 r h o C\right)\right) k s p a^{4}+\mathrm{O}\left(k s p a^{6}\right) \\
& >\text { numTnSS_kspa2 }:=\operatorname{simplify}((\operatorname{coeff}(\text { numTnSS_Serkspa, kspa, 2) ) ) } \\
& \text { numTnSS_kspa2 }:=\frac{1}{2 n+3}\left(2 k s a ^ { 4 } r h o P ^ { 2 } \left(2 k s a n^{4} j n p \_j n k s a+4 k s a n^{3} j n p \_j n k s a+2 n^{5}\right.\right. \\
& \left.\left.+k s a n^{2} j n p \_j n k s a+6 n^{4}-4 k s a n j n p \_j n k s a+5 n^{3}-3 k s a j n p \_j n k s a-3 n^{2}-7 n-3\right)\right) \\
& >\text { numTnSS_kspa2facs }:=\operatorname{simplify}\left(\operatorname{expand}\left(\operatorname{collect}\left(\frac{n u m T n S S \_k s p a 2 \cdot(2 \cdot n+3)}{2 \cdot k s a^{4} \cdot r h o P^{2}}, j n p \_j n k s a\right)\right)\right) \\
& n u m T n S S \_k s p a 2 f a c s:=2 k s a n^{4} j n p \_j n k s a+4 k s a n^{3} j n p \_j n k s a+2 n^{5}+k s a n^{2} j n p \_j n k s a+6 n^{4} \\
& -4 k s a n j n p \_j n k s a+5 n^{3}-3 k s a j n p \_j n k s a-3 n^{2}-7 n-3 \\
& >\text { numTnSS_kspa2facsSimpl }:=\left(2 \cdot n^{4}+4 \cdot n^{3}+n^{2}-4 \cdot n-3\right) \cdot\left(k s a \cdot j n p \_j n k s a+n+1\right) \\
& \text { numTnSS_kspa2facsSimpl }:=\left(2 n^{4}+4 n^{3}+n^{2}-4 n-3\right)(\text { ksajnp_jnksa }+n+1) \\
& >\text { numTnSS_kspa2facsSimpl }:=(n-1) \cdot(n+1) \cdot\left(2 \cdot n^{2}+4 \cdot n+3\right) \cdot\left(k s a \cdot j n p \_j n k s a+n+1\right) \\
& \text { numTnSS_kspa2facsSimpl }:=(n-1)(n+1)\left(2 n^{2}+4 n+3\right)\left(k s a j n p \_j n k s a+n+1\right) \\
& \text { ***************This (above) is the simplified expression for the first term in the numerator of } \\
& \text { LnSS_combined with factors in line } 62 \text {. } \\
& >\operatorname{expand}(\text { numTnSS_kspa2facs - numTnSS_kspa2facsSimpl) } \\
& \text { \# check that this agrees with the original. } \\
& 0 \\
& >\text { numTnSS_kspa4 }:=\operatorname{simplify}((\operatorname{coeff}(\text { numTnSS_Serkspa, kspa, 4 ) ) } \\
& \text { numTnSS_kspa4 }:=-\frac{1}{(2 n+3)\left(4 n^{2}+16 n+15\right)}\left(k s a ^ { 2 } \text { rhoP } \left(8 k s a^{3} n^{4}\right.\right. \text { rhoC jnp_jnksa } \\
& +12 k s a^{3} n^{4} \text { rhoP jnp_jnksa }+36 k s a^{3} n^{3} \text { rhoCjnp_jnksa }+52 k s a^{3} n^{3} \text { rhoP jnp_jnksa } \\
& -8 k s a^{2} n^{5} r h o C+12 k s a^{2} n^{5} r h o P+46 k s a^{3} n^{2} r h o C j n p \_j n k s a \\
& +78 k s a^{3} n^{2} \text { rhoP jnp jnksa }-60 k s a^{2} n^{4} r h o C+64 k s a^{2} n^{4} r h o P \\
& +24 \mathrm{ksan}^{4} \text { rhoCjnp_jnksa }+15 \mathrm{ksa}^{3} n \text { rhoCjnp_jnksa }+53 \mathrm{ksa}^{3} \text { n rhoP jnp_jnksa } \\
& -178 \mathrm{ksa}^{2} \mathrm{n}^{3} \mathrm{rhoC}+130 \mathrm{ksa}^{2} \mathrm{n}^{3} \mathrm{rhoP}+96 \mathrm{ksan} \mathrm{n}^{3} \text { rhoCjnp_jnksa }+24 n^{5} \text { rhoC } \\
& +15 k s a^{3} \text { rhoP jnp_jnksa }-261 k s a^{2} n^{2} \text { rhoC }+131 k s a^{2} n^{2} r h o P \\
& +66 k s a n^{2} \text { rhoC jnp jnksa }+120 n^{4} \text { rhoC }-183 k s a^{2} n \text { rhoC }+68 k s a^{2} n \text { rhoP } \\
& -96 k s a n \text { rhoC jnp_jnksa }+162 n^{3} \text { rhoC }-45 k s a^{2} r h o C+15 k s a^{2} r h o P \\
& \text { - } 90 \text { ksa rhoC jnp_jnksa }-30 n^{2} \text { rhoC }-186 \text { n rhoC }-90 \text { rhoC) ) } \\
& >\text { numTnSS_kspa4facs }:=\operatorname{collect}\left(\operatorname{expand}\left(\frac{n u m T n S S \_k s p a 4 \cdot(2 \cdot n+3) \cdot\left(4 \cdot n^{2}+16 \cdot n+15\right)}{k s a^{2} \cdot r h o P}\right)\right. \text {, }
\end{aligned}
$$


rhoP)

numTnSS_kspa4facs $:=\left(-12 k s a^{3} n^{4} j n p \_j n k s a-52 k s a^{3} n^{3} j n p \_j n k s a-12 k s a^{2} n^{5}\right.$

$-78 k s a^{3} n^{2} j n p \_j n k s a-64 k s a^{2} n^{4}-53 k s a^{3} n j n p \_j n k s a-130 k s a^{2} n^{3}-15 k s a^{3} j n p \_j n k s a$

$\left.-131 k s a^{2} n^{2}-68 k s a^{2} n-15 k s a^{2}\right) r h o P-8 k s a^{3} n^{4}$ rhoCjnp jnksa

$-36 k s a^{3} n^{3}$ rhoC jnp_jnksa $+8 k s a^{2} n^{5}$ rhoC $-46 k s a^{3} n^{2}$ rhoC jnp_jnksa

$+60 \mathrm{ksa}^{2} \mathrm{n}^{4} \mathrm{rhoC}-24 \mathrm{ksa} \mathrm{n}^{4}$ rhoCjnp_jnksa $-15 \mathrm{ksa} \mathrm{a}^{3} \mathrm{n}$ rhoCjnp_jnksa

$+178 k s a^{2} n^{3}$ rhoC $-96 k s a n^{3}$ rhoC jnp_jnksa $-24 n^{5}$ rhoC $+261 k s a^{2} n^{2}$ rhoC

$-66 \mathrm{ksan}^{2}$ rhoCjnp_jnksa $-120 n^{4}$ rhoC $+183 \mathrm{ksa}^{2}$ n rhoC $+96 \mathrm{ksa} n$ rhoC jnp_jnksa

$-162 n^{3}$ rhoC $+45 k s a^{2}$ rhoC $+90 k s a r h o C j n p \_j n k s a+30 n^{2} r h o C+186 n$ rhoC

+90 rhoC

Now attempt to extract similar terms to those appearing in the $n=1$ coefficient - see 2017 JASA paper. Work out the factors appearing in front of the Bessel function terms by hand by taking all the rhoP terms.

$>$ numTnSS_kspa4facsRed $:=$ expand $\left(\right.$ numTnSS_kspa4facs $-\left(-(n+1) \cdot\left(12 \cdot n^{3}+40 \cdot n^{2}+38 \cdot n\right.\right.$ $+15) \cdot k s a^{2} \cdot r h o P \cdot(k s a \cdot j n p$ jnksa $\left.\left.+(n+1))\right)\right)$

numTnSS_kspa4facsRed $:=-8 \mathrm{ksa} \mathrm{n}^{4}$ rhoCjnp_jnksa $-36 \mathrm{ksa} \mathrm{n}^{3}$ rhoCjnp_jnksa

$+8 k s a^{2} n^{5}$ rhoC $-46 k s a^{3} n^{2}$ rhoC jnp_jnksa $+60 k s a^{2} n^{4}$ rhoC $-24 k s a n^{4}$ rhoC jnp_jnksa

(68)

$-15 \mathrm{ksa}^{3} n$ rhoC jnp_jnksa $+178 \mathrm{ksa}^{2} n^{3}$ rhoC $-96 \mathrm{ksa} n^{3}$ rhoC jnp_jnksa $-24 n^{5}$ rhoC

$+261 \mathrm{ksa}^{2} n^{2}$ rhoC $-66 k s a n^{2}$ rhoCjnp_jnksa $-120 n^{4}$ rhoC $+183 \mathrm{ksa}^{2} \mathrm{n} \mathrm{rhoC}$

+ 96 ksa n rhoC jnp_jnksa $-162 n^{3}$ rhoC $+45 k s a^{2}$ rhoC +90 ksa rhoC jnp jnksa

$+30 n^{2} r h o C+186 n r h o C+90 r h o C$

Tere are no rhoP terms remaining. Now take a similar term in rhoC to get the (rhoP-rhoC) term as in the $n=1$ coefficient

$>$ numTnSS_kspa4facsRed $:=\operatorname{simplify}\left(\right.$ expand $\left(\right.$ numTnSS_kspa4facsRed $-\left((n+1) \cdot\left(12 \cdot n^{3}+40\right.\right.$ $\left.\left.\left.\left.\cdot n^{2}+38 \cdot n+15\right) \cdot k s a^{2} \cdot r h o C \cdot\left(k s a \cdot j n p \_j n k s a+(n+1)\right)\right)\right)\right)$

numTnSS_kspa4facsRed $:=-20 \mathrm{ksa}^{3} \mathrm{n}^{4}$ rhoC jnp_jnksa $-88 \mathrm{ksa} \mathrm{n}^{3}$ rhoC jnp_jnksa

$-4 k s a^{2} n^{5}$ rhoC $-124 k s a^{3} n^{2}$ rhoCjnp_jnksa $-4 k s a^{2} n^{4}$ rhoC $-24 k s a n^{4}$ rhoC jnp_jnksa

$-68 k s a^{3} n$ rhoCjnp_jnksa $+48 k s a^{2} n^{3}$ rhoC $-96 k s a n^{3}$ rhoCjnp_jnksa $-24 n^{5}$ rhoC

$-15 k s a^{3}$ rhoCjnp_jnksa $+130 k s a^{2} n^{2} r h o C-66 k s a n^{2}$ rhoCjnp_jnksa $-120 n^{4}$ rhoC

$+115 \mathrm{ksa}^{2} \mathrm{n}$ rhoC $+96 \mathrm{ksan}$ rhoCjnp_jnksa $-162 \mathrm{n}^{3}$ rhoC $+30 \mathrm{ksa}^{2} \mathrm{rhoC}$

+90 ksarhoCjnp_jnksa $+30 n^{2}$ rhoC $+186 n$ rhoC +90 rhoC

Now take a similar term in rhoC by with the other combination of jnp_jnksa to complete the betajS term (JASA2017 paper) in $(2 \mathrm{n}+1)$

$>$ numTnSS_kspa4facsRed $:=\operatorname{simplify}\left(\operatorname{expand}\left(\right.\right.$ numTnSS_kspa4facsRed $-\left(-(2 \cdot n+1) \cdot\left(12 \cdot n^{3}\right.\right.$

$$
\left.\left.\left.\left.+40 \cdot n^{2}+38 \cdot n+15\right) \cdot k s a^{2} \cdot \operatorname{rhoC} \cdot\left(k s a \cdot j n p \_j n k s a-n\right)\right)\right)\right)
$$

numTnSS_kspa4facsRed $:=4 \mathrm{ksa}^{3} \mathrm{n}^{4}$ rhoCjnp_jnksa $+4 \mathrm{ksa} \mathrm{n}^{3} \mathrm{n}^{3}$ rhoC jnp_jnksa

$-28 k s a^{2} n^{5}$ rhoC $-8 k s a^{3} n^{2}$ rhoC jnp_jnksa $-96 k s a^{2} n^{4}$ rhoC $-24 k s a n^{4}$ rhoC jnp_jnksa 


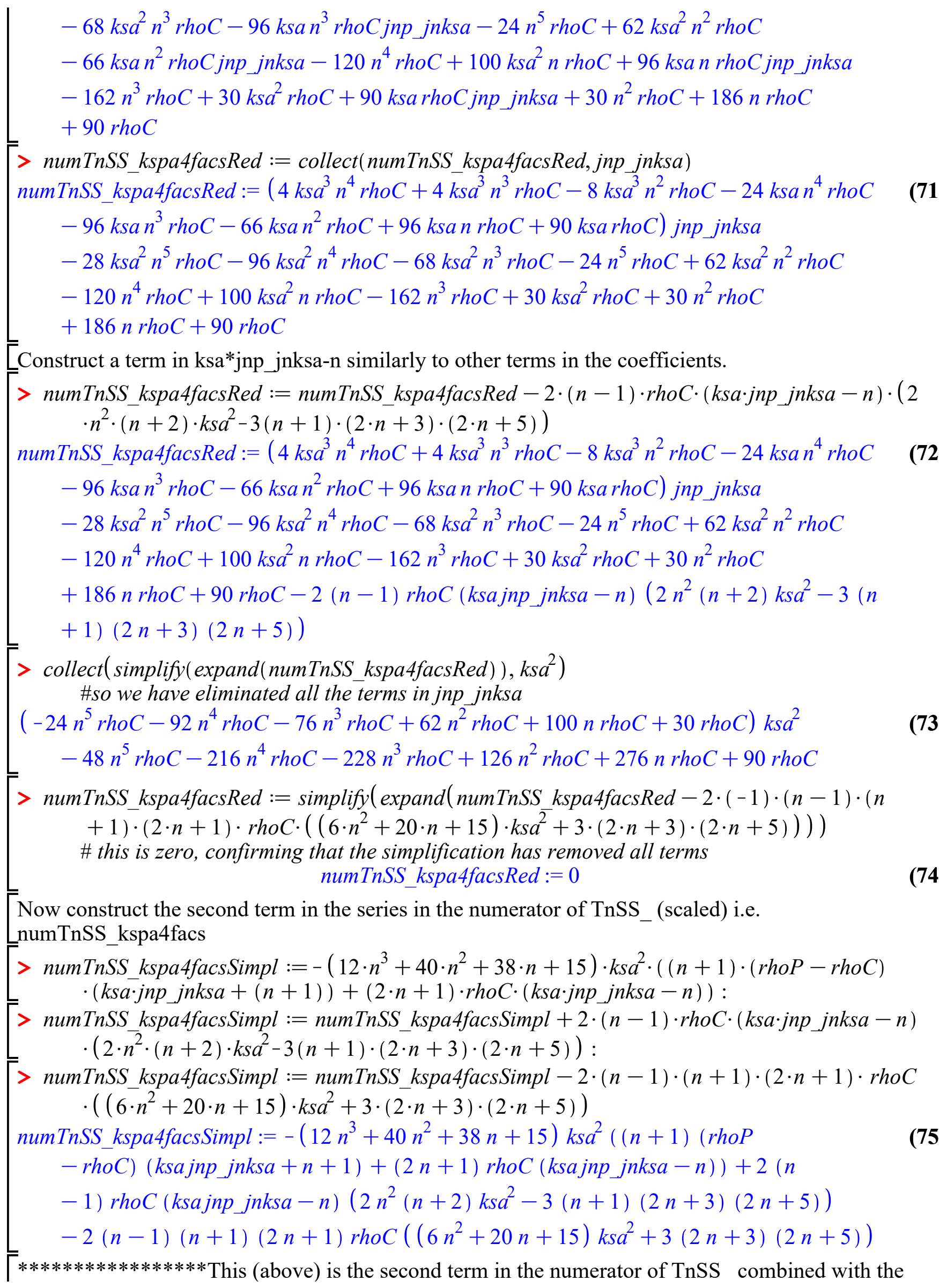


Lfactors in line 67.

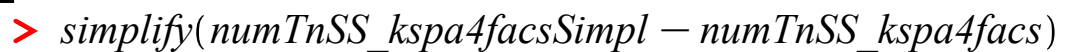

\# check that it agrees with the original. Confirms that this simplified version is correct.

$* * * * * * * * * * * * * * * * * * * * * * * * * * * * * * * * * * * * * * * * * * * * * * * * * * * * * * * * * * * * * * * * * * * * * * * * * * * *$

The derivation of the expressions for the scaled coefficients are now complete - see results above.

$* * * * * * * * * * * * * * * * * * * * * * * * * * * * * * * * * * * * * * * * * * * * * * * * * * * * * * * * * * * * * * * * * * * * * * * * * * * * * * * * * * * * *$

$* * * * * * * * * * * * * * * * * * * * * * * * * * * * * * * * * * * * * *$

Full expressions for the unscaled coefficients can be obtained using the expansions for hn and jn for small arguments for kca terms, and not expanding those for ksa.

For $\mathrm{n}>=2$

$$
\begin{aligned}
& >T n C C:=\frac{\left(k s a \cdot h n p \_h n k s a-n\right) \cdot n}{(n+1) \cdot\left(k s a \cdot h n p \_h n k s a+(n+1)\right)} \cdot \frac{j n k c a}{\text { hnkca }} \\
& T n C C:=\frac{\left(k s a h n p \_h n k s a-n\right) n j n k c a}{(n+1)\left(k s a h n p \_h n k s a+n+1\right) h n k c a} \\
& >\operatorname{TnCS}:=-\frac{(2 \cdot n+1)}{(n+1) \cdot\left(k s a \cdot h n p \_h n k s a+(n+1)\right)} \cdot \frac{j n k c a}{h n k s a} \\
& T n C S:=-\frac{(2 n+1) j n k c a}{(n+1)\left(k s a h n p \_h n k s a+n+1\right) h n k s a} \\
& >\text { TnSC }:=\frac{i \cdot n}{k s a \cdot\left(k s a \cdot h n p \_h n k s a+(n+1)\right) h n k s a \cdot j n k s a} \cdot \frac{j n k s a}{h n k c a} \\
& T n S C:=\frac{i n}{k s a\left(k s a h n p \_h n k s a+n+1\right) h n k s a h n k c a} \\
& >T n S S:=-\frac{\left(k s a \cdot j n p \_j n k s a+(n+1)\right)}{\left(k s a \cdot h n p \_h n k s a+(n+1)\right)} \cdot \frac{j n k s a}{h n k s a} \\
& T n S S:=-\frac{\left(k s a j n p \_j n k s a+n+1\right) j n k s a}{\left(k s a h n p \_h n k s a+n+1\right) h n k s a}
\end{aligned}
$$

Now check out the orders of all these coefficients with kca - and possibly get the leading term of jn and hn from the expansions above (but need to get the first term of $1 / \mathrm{hn}$ )

$$
\begin{aligned}
& \Gamma \text { jnxx } \\
& \frac{x^{n}\left(1-\frac{x^{2}}{4 n+6}+\frac{1}{8} \frac{x^{4}}{(2 n+3)(2 n+5)}+\mathrm{O}\left(x^{6}\right)\right)}{f a c_{j} j n} \\
& >\text { jnkca_lead }:=\frac{1}{f a c \_j n} \# \text { times } k c a^{n} \text { and } f a c j n=1 \cdot 3 \cdot \ldots(2 n+1) \\
& \text { jnkca_lead }:=\frac{1}{f a c \_j n} \\
& \Gamma>\text { jnkca }:=\text { jnkca_lead } \\
& j n k c a:=\frac{1}{f a c j n}
\end{aligned}
$$




$$
\begin{aligned}
& i^{-n-1}(2 n) ! i^{n}\left(1-i x-\frac{(n-1) x^{2}}{2 n-1}\right) \\
& n !(2 x)^{n} \\
& >\text { hnkca }:=-\frac{i \cdot(2 \cdot n) !}{n ! \cdot 2^{n}} \text { \#times } k_{c a}{ }^{-(n+1)} \text { leading term only } \\
& h n k c a:=-\frac{i(2 n) !}{n ! 2^{n}} \\
& \text { TnCC_lead }:=-\frac{\left(k s a h n p \_h n k s a-n\right) n n ! 2^{n}}{(n+1)\left(k s a h n p \_h n k s a+n+1\right) f a c \_j n i(2 n) !} \\
& \text { TnCS_lead }:=-\frac{2 n+1}{(n+1)\left(k s a h n p \_h n k s a+n+1\right) f a c \_j n h n k s a} \\
& T n S C \_l e a d:=-\frac{n n ! 2^{n}}{k s a\left(k s a h n p \_h n k s a+n+1\right) h n k s a(2 n) !} \\
& T n S S \_l e a d:=-\frac{\left(k s a j n p \_j n k s a+n+1\right) j n k s a}{\left(k s a h n p \_h n k s a+n+1\right) h n k s a} \\
& n:=1 \\
& T n C S \_n 1:=\frac{3(-r h o P+r h o C)}{k s a r h o C h n p \_h n k s a+2 k s a r h o P h n p \_h n k s a-7 \text { rhoC }+4 \text { rhoP }} \\
& >T n S C \_n 1:=\operatorname{simplify}\left(\frac{n u m T n S C \_k s p a 4 f a c s \text { Simpl }}{\text { denTnCC_kspa4facsSimpl }}\right) \\
& T n S C \_n 1:= \\
& \frac{1}{105} \mathrm{I}\left[-210 k s a^{2}(-r h o P+r h o C)\right]
\end{aligned}
$$

\footnotetext{
ksa jnksa hnksa (ksa rhoChnp_hnksa +2 ksarhoP hnp_hnksa -7 rhoC +4 rhoP)

$>T n S S \_n 1:=\operatorname{simplify}\left(\frac{\text { numTnSS_kspa4facsSimpl }}{\operatorname{denTnCC\_ kspa4facsSimpl}}\right)$
} 


$$
T n S S \_n 1:=-\frac{k s a r h o C j n p \_j n k s a+2 \text { ksarhoP jnp_jnksa }-7 \text { rhoC }+4 \text { rhoP }}{k s a r h o C h n p \_h n k s a+2 \text { ksarhoP hnp_hnksa }-7 \text { rhoC }+4 \text { rhoP }}
$$

These are the scaled coefficients for $\mathrm{n}=1$, these agree with Pinfield and Forrester, Journal of the Acoustical Society of America, 141, 649 (2017); doi: 10.1121/1.4974142 